Bulletin of the Natural History Museum, 2015, 8: 118-167.

Received 08 Jun 2015; Accepted 23 Dec 2015.

doi: $10.5937 /$ bnhmb1508118D

UDC: 069.51:597.6.082.5(497.11)

Original scientific paper

\title{
THE BATRACHOLOGICAL COLLECTIONS OF THE INSTITUTE FOR BIOLOGICAL RESEARCH "SINIŠA STANKOVIĆ", UNIVERSITY OF BELGRADE
}

\author{
GEORG DŽUKIĆ ${ }^{1}$, MILENA CVIJANOVIĆ ${ }^{1 *}$, ALEKSANDAR UROŠEVIĆ ${ }^{1}$, \\ TANJA D. VUKOV ${ }^{1}$, NATAŠA TOMAŠEVIĆ KOLAROV ${ }^{1}$, MAJA SLIJEPČEVIĆ ${ }^{1}$, \\ ANA IVANOVIĆ ${ }^{2}$, MiLOŠ L. KALEZIĆ ${ }^{1}$ \\ ${ }^{1}$ Institute for Biological Research "Siniša Stanković”, Bulevar Despota Stefana \\ 142, 11000 Belgrade, Serbia, University of Belgrade, \\ e-mail: milena.cvijanovic@ibiss.bg.ac.rs \\ ${ }^{2}$ Institute of Zoology, Faculty of Biology, Studentski trg 16, 11000 Belgrade, \\ Serbia, University of Belgrade
}

This paper presents the information on amphibians specimens deposited in the collections of the Institute for Biological research "Siniša Stanković", University of Belgrade. It contains 33 amphibian species collected mostly in the territory of the former SFR of Yugoslavia, particularly in Serbia.

Key words: amphibians, collection

\section{INTRODUCTION}

Batrachological collection of the Institute for Biological research "Siniša Stanković", Belgrade has a very long history. Some of the oldest specimens were collected more than a century ago, the oldest being from 
1900. The important phase of collecting specimens was during late professor Milutin Radovanovic's lifetime (1900-1968). His work in herpetology, including material that he collected, was foundation for the further work in herpetology in Serbia as well as the batrachological and herpetological collections. The material collected and organized by him, including valuable specimens of Ichtyosaura alpestris from Montenegro and Bosnia and Herzegovina, was previously deposited in the collection of the Natural History Museum in Belgrade and later transferred to the Institute for Biological research "Siniša Stanković". Another important contributor was late professor Gojko Pasuljević (1931-1997) who collected specimens from the province of Kosovo and Metohija. After 1968, the intensive collecting of specimens began, with large population samples and different ontogenetic stages deposited in the herpetological collection.

The last time that this collection was published in its entirety was 43 years ago (Džukić 1972). Since then, a vast amount of samples have been added to the collection, but with no systematic efforts to sort and catalogize specimens, except adding collection numbers.

This Batrachological collection contains of 30288 specimens from 18 countries. Most of the specimens were collected in the territory of former Yugoslavia, with territories of Serbia and Montenegro being especially well covered. This collection is also enriched with material from various countries including Turkey, Iraq, France and United States of America among others (Fig. 1).
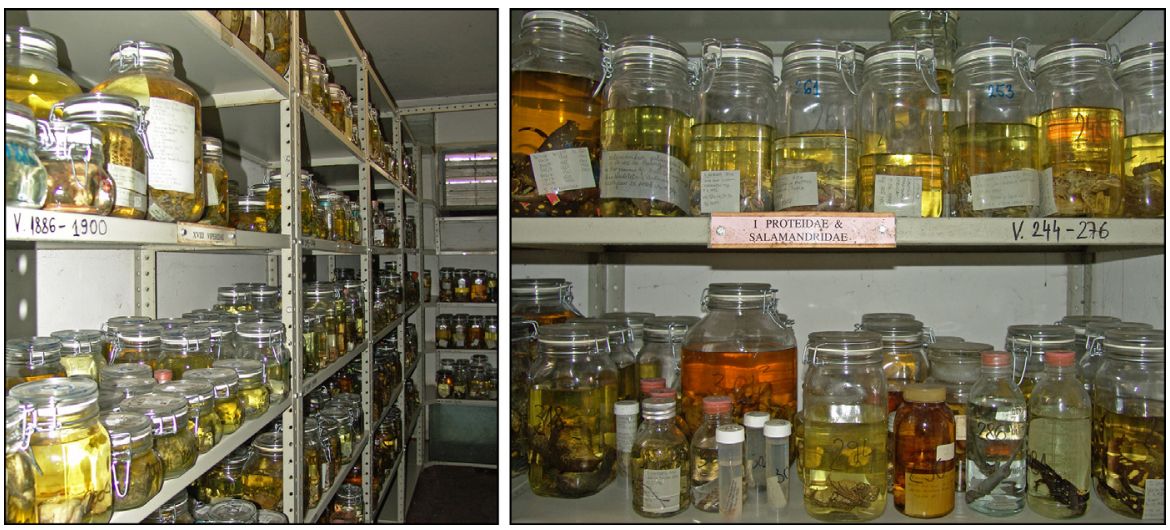

Fig. 1. - Batrachological collection of the Institute for Biological research "Siniša Stanković", University of Belgrade, Belgrade, Serbia.

This collection consists of several collections named by the batrachologist who considerably enlarge number of specimens, as well as distribution records. These collections are as followed: Milutin Radovanović, Gojko Pasuljević, Georg Džukić and Ljiljana Tomović with her collaborate. 


\section{MATERIALS AND METHODS}

The collection of amphibians of the Institute for Biological research "Siniša Stanković" consists of batrachological materials preserved in $75 \%$ ethanol. Some specimens were previously stored in formaldehyde, and transferred to $75 \%$ ethanol. All materials are stored in labeled jars or plastic sample tubes. During the period of 5 years (from 2011 until 2015) the complete sorting, conservation and inventarisation of the collection has been conducted, and new labels were added to the old ones with a new collection number for each jar or sample tube.

Identification was done according to the standard herpetological literature (e.g. Arnold \& Ovenden 2002) for the majority of species. Due to difficulties in determination of some species, species identification of some groups was mainly based on spatial distribution of presumably diagnostic morphological traits: diagnostic allozyme characters for green frogs (Krizmanić 2008, Krizmanić \& Ivanović 2010) and morphological and genetic analyses for Triturus cristatus s.l. (e.g. Kalezić et al. 1997, Wielstra et al. 2013).

Detailed information of collection are given in Online Appendix.

\section{RESULTS \\ Catalogue of taxa and specimens}

\section{Anura}

BUFONIDAE

\section{Bufo bufo}

S.l. (leg. anonim., s.d., 304, n.spec. = 3); (305); 318); (320); 532); (539); 549); (695); 712); (725); 1312); (1433); 1495); (1515); 1896); (leg. G. Džukić s.d., 990, n.spec. = 1); (1304); 1337); (1401); (leg. P. Durbešić 18-jul-1980, 2221, n.spec. = 2); Albania Kruja: Derven (leg. G. Džukić, 02-jun-1972, 552, n.spec. = 1); Bosnia and Herzegovina Mt. Igman (leg. G. Džukić, 01-aug-1974, 1281, n.spec. = 1); Mt. Zelengora: Gornje Bare lakes (leg. G. Džukić 13-may-1983, 694/2, n.spec. = 4); Tjentište (v.) (leg. G. Džukić 13-may-1983, 694/1, n.spec. = 4); Trebinje: Ljubovo (v.) (leg. G. Pasuljević 01-sep-1978, 2795, n.spec. = 2); Croatia Baranja: Batsiget (leg. J. Mikuška, 01-apr-1971, $669-2$, n.spec. =1); Bilje (v.) (leg. J. Mikuška 30-oct-1970, $669-2$, n.spec. = 1); Tikveš (v.) (leg. J. Mikuška 31-aug-1970, 669 - 2, n.spec. =1); Gorski Kotar: Crni Lug (v.) (leg. anonim. 16-jul-1964, 1156, n.spec. =1); Novigrad, Žužići (v.) (leg. G. Džukić 09-jun-1975, 1403, n.spec. =1); Koprivnica: Peteranec (v.) (leg. anonim. 05-apr-1988, 715, n.spec. =1); Rijeka: Kukuljanovo (v.) (leg. G. Džukić 25-apr-1981, 1434, n.spec. = 1); Velika Gorica: Turopolje, Orle (v.) (leg. G. Džukić 23-apr1981, 1434, n.spec. =1); Macedonia Mt. Jablanica: Gornja Belica (v.) (leg. G. Džukić, 23-sep1996, 2140, n.spec. = 1); Podgorečko lake (leg. G. Džukić, R.S. Petkovski 21-sep-1996, 2285, n.spec. = 1); Višni (v.) (leg. G. Džukić 23-sep-1996, 1425, n.spec. = 1); Prespa lake: Ezerani (v.) (leg. anonim. 07-may-1997, 987, n.spec. = 2); (2165); Prilep: Gradsko (leg. anonim. 15-apr-1972, 535, n.spec. = 1); Montenegro Berane [“Ivangrad”] (leg. G. Pasuljević, 13-jul-1980, 2795, n.spec. = 3); 
Polica (leg. R. Celić 04-apr-1983, 1896, n.spec. = 3); Trpeška Rijeka (leg. I. Skenderović 20-aug1987, 1896, n.spec. = 1); Danilovgrad: Džegovica, Porinta (leg. M. Kalezić, G. Džukić, M. Marković 19jun-2004, 2286, n.spec. =1); Mt. Bjelasica: Biogradsko lake (leg. G. Pasuljević 16-jun-1970, 2795 , n.spec. = 1); Mt. Durmitor (leg. anonim. s.d., 682, n.spec. =1); (1310); 1314); (1400); (1630); Barno lake (leg. J.J. Purger 07-jul-1989, 1309, n.spec. = 1); Crepulj Poljana (leg. P. Jakšić 11-jul-1987, 1309, n.spec. = 1); Crno lake (leg. anonim. 01-aug-1959, 1624, n.spec. = 5); Žabljak (leg. P. Jakšić 02-jul-1987, 1309, n.spec. = 2); Mt. Njegoš: Dubočke (v.) (leg. G. Pasuljević 10-mar-1976, 2795, n.spec. =1); Srijede (v.) (leg. G. Džukić 11-jun-1973, 548, n.spec. =2); Mt. Rumija: Sutorman (v.) (leg. M. Kalezić, G. Džukić, M. Marković 02-jun-2003, 1415, n.spec. = 1); Plav (leg. G. Pasuljević 11-jul1976, 2795, n.spec. = 1); Podgorica: Ubli (v.), Liješta (v.), Roge puddle (leg. G. Džukić, I. Aleksić, M. Kalezić, D. Bejaković 06-apr-1992, 1880, n.spec. = 3); Rijeka Crnojevića: Orahovštica stream (leg. G. Džukić 10-mar-1974, 1332, n.spec. = 1); Skadarsko lake: Krajina, Livari (v.) (leg. J. Crnobrnja-Isailović, I. Aleksić 29-may-1996, 200, n.spec. = 1); Ulcinj: Velika Plaža beach (leg. G. Džukić, M. Kalezić, M. Denoel 08-jun-2005, 1382, n.spec. = 2); Virpazar: Orahovačko Polje (leg. G. Džukić, I. Aleksić, M. Kalezić, D. Bejaković 07-apr-1992, 1881, n.spec. = 2); Serbia (leg. anonim., s.d., 1465, n.spec. = 2); (1873); (leg. G. Pasuljević s.d., 2793, n.spec. = 8); Aleksinac: Ćićina (v.) (leg. G. Džukić, M. Kalezić 13-may-2002, 1424, n.spec. =1); Bela Crkva: Jaruga stream, Mrtva Nera (leg. G. Džukić, M. Kalezić 18-mar-2001, 2147, n.spec. = 1); Deliblato sands: Bela Crkva - Kovin, Dubovac (v.) (leg. G. Džukić, M. Kalezić 19-mar-2001, 1414, n.spec. = 5); Deliblato (v.), Kraljevica (leg. M. Kalezić, G. Džukić s.d., 246, n.spec. =7); Đurica, Stevanove Ravnice (leg. G. Džukić, M. Kalezić 18-mar-2001, 2142, n.spec. = 2); (leg. M. Kalezić, G. Džukić 22-aug-1998, 2030, n.spec. =1); Korn (leg. G. Džukić, M. Marković 29-apr-1980, 1439, n.spec. = 2); Mramorak (v.) (leg. G. Džukić, M. Kalezić 18mar-2005, 1083, n.spec. = 1); Kraljevac, Obzovik Bara (leg. G. Džukić, M. Kalezić 25-mar-2005, 1899, n.spec. = 10); Kraljevac, Obzovik Bara, Vodice churches (leg. G. Džukić, M. Kalezić 25-mar-2005, 2012, n.spec. =1); Zamfir Bara (leg. G. Džukić, M. Kalezić 04-jun-2000, 1100, n.spec. =10); Gnjilane: Kosovska Kamenica (leg. G. Pasuljević 19-aug-1979, 2794, n.spec. = 3); Grdelička gorge: Predejane (v.) (leg. G. Džukić, M. Kalezić 20-apr-2007, 2156, n.spec. = 2); Kladovo: Lolića Rit (leg. G. Džukić, M. Kalezić 17-may-2000, 1409, n.spec. = 1); Vrbica (v.) (leg. G. Džukić, M. Kalezić 25-apr2009, 1064, n.spec. = 12); Kosovska Mitrovica: Banjska (v.) (leg. G. Pasuljević 07-mar-1978, 2792, n.spec. = 14); Leposavić (leg. G. Pasuljević 03-jun-1973, 2794, n.spec. = 1); Zubin Potok, Lučka river (leg. G. Pasuljević 01-oct-1976, 2795, n.spec. =1); Kuršumlija: Pljakovo (v.) (leg. G. Pasuljević 03-jul-1979, 2795, n.spec. = 1); Leskovac: Milanovo (v.) (leg. G. Džukić, M. Kalezić 01-sep-2000, 1246, n.spec. = 1); Turekovac (v.) (leg. G. Džukić, M. Kalezić 01-sep-2000, 1246, n.spec. = 1); Loznica: Lešnica (v.) (leg. anonim. 23-mar-1991, 1528, n.spec. =1); Mt. Avala: Čarapićev Brest (leg. B. Žiljak 01-aug-1996, 2067, n.spec. =1); Mt. Besna Kobila: Kriva Feja (v.) (leg. G. Džukić, M. Kalezić 17-jun-2006, 1137, n.spec. = 19); Mt. Fruška Gora: Testera (leg. G. Džukić 06-jun-1976, 1417, n.spec. = 3); Mt. Maljen: Divčibare (leg. J. Crnobrnja-lsailović 26-jul-1999, 2029, n.spec. = 1); (leg. M. Kalezić 13-mar-1994, 2042, n.spec. = 2); Mt. Ostrozub: Ruplje (v.), Bankovci (v.), Ivanova Bara (leg. G. Džukić, M. Kalezić 31-mar-2007, 2163, n.spec. =3); Mt. Tara (leg. G. Džukić 11-jun-1969, 725, n.spec. =1); Miloševac (leg. G. Džukić 16-may-1972, 725, n.spec. =1); Rajevac (leg. G. Džukić, M. Kalezić 12-jun-2001, 1426, n.spec. = 1); Zaovine (v.), Beli Rzav lake (leg. G. Džukić 21-oct-1973, 1465, n.spec. = 1); Mt. Vršačke Planine: hunting lodge (leg. G. Džukić, M. Kalezić 12-apr-2004, 2141, n.spec. = 1); Mesić (v.) (leg. G. Džukić, M. Kalezić 27-mar-2004, 1391, n.spec. = 1); Mts ŠarPlanina (leg. G. Pasuljević 1971, 2794, n.spec. =1); Dragaš, Rapča (v.) (leg. G. Pasuljević 22-may1980, 2795, n.spec. = 1); Gora, lakes (leg. G. Džukić, M. Kalezić, J. Crnobrnja-Isailović 01-jul-1995, 559, n.spec. = 1); Pančevo: Ivanovo (v.) (leg. G. Džukić, M. Kalezić 02-apr-2002, 1424, n.spec. = 1); Peć (leg. G. Pasuljević 21-sep-1976, 2793, n.spec. = 3); Dobruša (v.) (leg. G. Pasuljević 11-sep-1978, 2795, n.spec. = 1); Istok (v.) (leg. G. Džukić 06-jun-1977, 1417, n.spec. = 1); Vitomirica (v.) (leg. G. Pasuljević 15-jul-1978, 2794, n.spec. = 4); Priboj (leg. G. Pasuljević 15-sep-1979, 2795, n.spec. = 1); Priština (leg. Z. Krivošej, S. Ristić 15-mar-1981, 1896, n.spec. =3); Mt. Grmija (leg. B. Bajčetić 22-may1984, 1896, n.spec. = 1); (leg. G. Pasuljević 12-mar-1981, 2794, n.spec. = 2); Slatina (v.) (leg. G. Džukić 05-jul-1969, 725, n.spec. = 1); Prizren (leg. G. Pasuljević 29-jul-1979, 2794, n.spec. = 1); Prokuplje: Konjuša (v.) (leg. G. Pasuljević 13-sep-1975, 2795, n.spec. = 1); Trgovište: Čivčije (v.) (leg. anonim. 01-apr-2004, 2120, n.spec. = 1); Trstenik: Počekovina (v.) (leg. K. Ljubisavljević 25-jul-1997, 244, n.spec. = 1); Uroševac (leg. G. Pasuljević 28-may-1979, 2794, n.spec. = 4); 
Gadimlje (v.) (leg. G. Pasuljević 09-jun-1979, 2794, n.spec. =1); Nerodimlje (v.) (leg. G. Pasuljević 14-jun-1980, 2795, n.spec. = 1); Valjevo: Petnica (v.) (leg. Ž. Marjanović 01-jul-1999, 1104, n.spec. $=$ 2); Veliko Gradište: Kumane (v.) (leg. M. Kalezić, G. Džukić 14-apr-1994, 2037, n.spec. = 2); Ostrovo (v.) (leg. D. Talev 01-sep-1994, 1455, n.spec. = 1); Vlasina (leg. G. Džukić, M. Kalezić 21-apr2007, 2164, n.spec. = 5); Okruglica (v.), Čićin Grad (leg. G. Džukić, M. Kalezić 26-sep-2008, 2180, n.spec. = 1); Vlasotince: Svođe (v.) (leg. G. Džukić, M. Kalezić 26-sep-2008, 2180, n.spec. =1); Slovenia Murska Sobota: Radenci (v.), Turjanci (v.) (leg. anonim., 01-apr-1988, 1398, n.spec. = 2); Novo Mesto: Mirna (v.) (leg. B. Kryštufek 23-apr-1986, 726, n.spec. =1); Sežana - Koper: Petrinje (v.) (leg. J. Gregori 07-may-1986, 726, n.spec. =1).

\section{Bufo viridis}

S.I. (leg. anonim., s.d., 304, n.spec. = 7); (305); 319); (331); 344); (675); 683); (1339); 1342); (1383); 1433); (1515); 1516); (1896); 2751); (leg. G. Džukić s.d., 1625, n.spec. = 13); (1874); Banjan (leg. anonim. 03-aug-1972, 1306, n.spec. = 2); Dobri Do (leg. G. Pasuljević 15-oct-1979, 2802, n.spec. = 1); Bosnia and Herzegovina Bosansko Grahovo: Korana stream (leg. anonim., 09-apr1989, 2662, n.spec. = 1); Mt. Maglić: Prijevor (leg. anonim. 09-sep-1970, 1885, n.spec. = 1); Sarajevo: Kijevo (v.), Železnica river (leg. G. Džukić 12-may-1983, 694/1, n.spec. =2); Croatia Baranja: Batina (v.) (leg. anonim., 09-apr-1972, 532, n.spec. = 1); Bilje (v.) (leg. J. Mikuška 30-oct1970, $669-2$, n.spec. = 1); Cres (ins.): Cres, near town (leg. N. Tvrtković 07-apr-1976, 705, n.spec. $=$ 1); Dugi Otok (ins.) (leg. B. Petrov 10-jul-1973, 548, n.spec. = 1); Mt. Velebit (leg. N. Tvrtković 31-jul1976, 1343, n.spec. = 1); Vis (ins.): Podšpilje (v.) (leg. G. Džukić, N. Tvrtković 08-oct-1975, 704, n.spec. = 6); Zagreb: Trnsko (leg. D. Pelić, G. Džukić 01-may-1975, 693, n.spec. =1); Greece Solun (leg. V. Vasilios, D. Tsizmu, 10-aug-1982, 1896, n.spec. =1); Macedonia Gevgelija (leg. G. Džukić, 09-nov-1972, 547, n.spec. =1); Kočani (leg. G. Džukić, S. Petkovski 23-jun-1995, 215, n.spec. =2); Mt. Galičica: Ohrid (leg. G. Džukić, B. Krištufek 22-sep-1996, 1412, n.spec. =4); Mt. Jablanica: Gornja Belica (v.) (leg. G. Džukić 23-sep-1996, 2140, n.spec. = 3); Višni (v.) (leg. G. Džukić 23-sep-1996, 1425, n.spec. =4); Negotino - Demir Kapija (leg. G. Džukić 07-nov-1973, 532, n.spec. =1); Prespa lake: Ezerani (v.) (leg. G. Džukić 07-may-1997, 2681, n.spec. =1); Strumica: Ilovica (v.) (leg. G. Džukić 24-jun-1998, 295, n.spec. =1); Montenegro Berane [“Ivangrad"] (leg. G. Pasuljević, 1979, 2791, n.spec. = 4); 16-mar-1981, 2801); (2802); Kalica (v.) (leg. I. Skenderović 20-aug-1987, 1896, n.spec. =2); Polica (leg. R. Celić 30-apr-1983, 1896, n.spec. =1); Mt. Durmitor (leg. anonim. s.d., 1314, n.spec. =1); Mt. Lukavica: Kapetanovo lake (leg. G. Džukić, M. Kalezić 23-aug-2002, 2521, n.spec. =7); Mt. Prekornica: Ponikvica, Ponikvica Brajovića (leg. G. Džukić, M. Kalezić 10-jun1997, 544, n.spec. =1); Mt. Žijovo: Bukumirsko lake, Đebeza (leg. M. Kalezić, G. Džukić, M. Marković 28-may-2003, 2297, n.spec. = 1); Rikavačko lake (leg. G. Džukić, M. Kalezić, A. Ivanović 20-sep-1991, 1839, n.spec. =6); Nikšić: Bijele Poljane (leg. G. Džukić, M. Marković, M. Kalezić 29-may-2003, 2362, n.spec. =1); Vir (v.) (leg. G. Džukić 11-jun-1973, 1625, n.spec. =1); Rijeka Crnojevića (leg. G. Pasuljević 30-oct-1977, 2791, n.spec. =1); (2802); Vilusi: Slivni Do (leg. G. Džukić 28-aug-1982, 547, n.spec. =1); Velimlje (v.) (leg. G. Pasuljević 15-jul-1976, 2802, n.spec. =2); Serbia (leg. anonim., s.d., 1876, n.spec. = 20); (1877); 1878); (1883); (leg. G. Pasuljević s.d., 2800, n.spec. = 19); Aleksandrovac: Vrbnica (v.) (leg. G. Džukić 02-jun-1977, 1877, n.spec. = 2); Babušnica (leg. G. Džukić, M. Kalezić 13-sep-2006, 1384, n.spec. =1); Bečej: Slano Kopovo [“Lesino Kopovo”] (leg. G. Džukić 18-apr-1990, 1440, n.spec. = 1); Bela Crkva: Jaruga stream (leg. G. Džukić, M. Kalezić 29-mar-2003, 2014, n.spec. =1); Bela Crkva - Kovin: Dubovac (v.) (leg. G. Džukić, M. Kalezić 19-mar-2001, 1414, n.spec. =1); Beograd (leg. G. Džukić 30-jul-1975, 1625, n.spec. =1); Borča (v.), Mali Zbeg (leg. anonim. 03-sep-1997, 1461, n.spec. = 1); (leg. L. Đurđević 05-sep-1999, 1413, n.spec. = 16); 08-sep-1999, 1390); 21-feb-2006, 2235); Košutnjak, Hajdučka Česma (leg. anonim. 11-jun-1970, 1878, n.spec. = 1); Krnjača (v.) (leg. M. Kalezić, G. Džukić 25-jun-2000, 2038, n.spec. = 4); Blace (leg. G. Pasuljević 20sep-1976, 2802, n.spec. = 3); Bor (leg. G. Pasuljević 10-sep-1979, 2791, n.spec. =1); Deliblato sands (leg. M. Kalezić, G. Džukić 04-jul-1998, 2034, n.spec. = 4); 21-aug-1998, 2031); Deliblato (v.) (leg. G. Džukić, M. Kalezić 18-mar-2005, 1083, n.spec. = 1); Deliblato (v.) - Mramorak (v.) (leg. M. Kalezić, G. Džukić 09-aug-1999, 2040, n.spec. = 1); Deliblatska Dolina (leg. anonim. 21-aug-1970, 1878, n.spec. = 1); Đurica, Stevanove Ravnice (leg. G. Džukić 22-may-1999, 1389, n.spec. = 2); (leg. G. 
Džukić, M. Kalezić 04-jul-1998, 2072, n.spec. = 1); Grebenac (v.) (leg. anonim. 02-jun-1970, 1878, n.spec. =1); Jasenovo (v.) (leg. G. Džukić 26-sep-2003, 1416, n.spec. = 1); Mali Pesak, Svinjska Plaža, Konjsko Groblje (leg. G. Džukić 26-sep-2003, 1416, n.spec. =2); Šumarak (v.) - Grebenac (v.) (leg. G. Džukić, M. Kalezić 19-mar-2001, 2142, n.spec. = 2); Šušara (v.) (leg. anonim. 01-may1970, 1878, n.spec. = 1); Utrine (leg. G. Džukić, M. Kalezić 01-aug-1999, 1388, n.spec. = 3); Đabin Salaš (leg. M. Kalezić, G. Džukić 09-aug-1999, 2035, n.spec. = 3); Vračev Gaj (v.) - Kovin (leg. G. Džukić, M. Kalezić 19-mar-2001, 2142, n.spec. = 1); Gnjilane (leg. D. Jović 05-jul-1982, 1896, n.spec. = 1); Gračaničko lake (leg. G. Pasuljević 10-may-1977, 2791, n.spec. = 1); Kosovska Kamenica (leg. G. Pasuljević 20-jul-1977, 2802, n.spec. =4); Sveti Jovan (leg. G. Pasuljević 20-aug-1977, 2791, n.spec. = 1); Jagodina: Donje Lanište (v.) (leg. M. Kalezić, G. Džukić 02-sep-2000, 2033, n.spec. = 2); Staro Lanište (v.), Nova Bara (leg. G. Džukić, M. Kalezić 14-may-2000, 1410, n.spec. = 1); Kladovo: Velesnica (v.) (leg. G. Džukić, M. Kalezić 13-jun-2006, 1419, n.spec. = 1); Klina: Jošanica (v.) (leg. G. Pasuljević 12-may-1977, 2802, n.spec. =1); Kosovo (leg. G. Pasuljević 10-nov1977, 2791, n.spec. = 12); Kosovska Mitrovica: Banjska (v.) (leg. G. Pasuljević 10-nov-1977, 2791, n.spec. = 19); 28-oct-1977, 2799); Leposavić (leg. G. Pasuljević 04-jun-1973, 2791, n.spec. = 2); 09jun-1978, 2802); Sočanica (v.) (leg. G. Pasuljević 18-jun-1979, 2791, n.spec. =1); Zvečan (v.) (leg. G. Pasuljević 15-may-1977, 2791, n.spec. =1); (2802); Kovin (leg. anonim. 16-aug-1970, 1878, n.spec. = 2); (leg. G. Džukić 1970, 1625, n.spec. = 1); Crna Bara - Deliblato (v.) (leg. G. Džukić, M. Kalezić 18mar-2005, 1067, n.spec. = 2); Gaj (v.) (leg. G. Džukić, M. Kalezić 18-mar-2005, 1060, n.spec. = 3); Skorenovac (v.) (leg. G. Džukić, M. Kalezić 25-mar-2005, 1046, n.spec. = 1); (2191); 23-may-2004, 2010); Kraljevo: Žiča (v.) (leg. S. Grujić 20-sep-1982, 1896, n.spec. = 1); Kruševac: Gornje Zleginje (v.) (leg. G. Pasuljević 10-jul-1976, 2791, n.spec. =1); (2801); Kuršumlija (leg. G. Pasuljević 07-jul-1979, 2801, n.spec. =1); 25-aug-1979, 2791); Rača (v.) (leg. G. Pasuljević 10-jun-1976, 2800, n.spec. =7); Leskovac: Belanovac (v.), Pašina Česma (leg. G. Džukić, M. Kalezić 01-sep-2000, 1246, n.spec. = 3); Bogojevac (leg. G. Džukić, M. Kalezić 01-sep-2000, 1106, n.spec. =1); Kumarevo (v.) (leg. G. Džukić, M. Kalezić 01-sep-2000, 1106, n.spec. = 1); Navalin (v.), Navalinsko Polje (leg. G. Džukić, M. Kalezić 01-sep-2000, 1106, n.spec. =1); Mt. Avala: Beli Potok (v.) (leg. anonim. 08-mar1990, 1043, n.spec. = 1); Mt. Fruška Gora: Zmajevac (leg. Z. Dundjerski 28-mar-1975, 1625, n.spec. =1); Mt. Rudnik (leg. D. Komatina 10-jun-1982, 1896, n.spec. =1); Mt. Tara (leg. G. Džukić 11-jun1969, 1877, n.spec. = 1); 21-jun-1973, 1878); Mt. Vršačke Planine (leg. G. Džukić 17-may-1976, 1625, n.spec. = 3); Mts Šar-Planina (leg. G. Pasuljević s.d., 2802, n.spec. =1); Niš: Mezgraja (v.) (leg. G. Pasuljević 08-oct-1977, 2802, n.spec. =1); Nova Varoš: Radoinjsko lake (leg. G. Džukić 11aug-1982, 1877, n.spec. = 2); Novi Pazar (leg. G. Pasuljević 14-aug-1976, 2802, n.spec. = 1); Obedska Bara: Obrež (v.), Ravenica (leg. G. Džukić, M. Kalezić 30-apr-1992, 1085, n.spec. = 5); Obrenovac: Umka (v.), Lipik hill (leg. M. Milenković 31-oct-1990, 1882, n.spec. = 1); Pančevo: Baranda (v.) (leg. G. Džukić 25-may-1974, 1877, n.spec. =2); Ivanovo (v.) (leg. D. Miljković 11-aug2006, 1075, n.spec. = 2); Peć (leg. G. Pasuljević 21-sep-1976, 2802, n.spec. =4); (leg. V. Šabović 20may-1979, 1164, n.spec. = 1); Đurakovac (v.) (leg. G. Pasuljević 15-oct-1978, 2802, n.spec. = 2); Istok (v.) (leg. G. Džukić 06-jun-1977, 1625, n.spec. = 1); Zatra (v.) (leg. G. Pasuljević 13-sep-1978, 2802, n.spec. = 1); Pirot: Jerma river gorge, Vlasi (v.) (leg. J. Crnobrnja-Isailović, I. Aleksić 01-jun1998, 313, n.spec. = 1); Krupac (v.), Krupačko Blato lake (leg. G. Džukić, M. Kalezić 14-may-2004, 1090, n.spec. =1); Pirot - Krupac (v.) (leg. G. Džukić 22-may-1977, 1625, n.spec. = 2); Priboj (leg. G. Pasuljević 16-nov-1979, 2791, n.spec. = 1); Priština (leg. G. Pasuljević 10-sep-1971, 2791, n.spec. = 1); 15-aug-1975, 2799); Čaglavica (v.) (leg. G. Pasuljević 15-jul-1976, 2802, n.spec. = 2); Kišnica (v.) (leg. Z. Stojanović 17-aug-1983, 1896, n.spec. =2); Kosovo Polje (leg. G. Pasuljević 25-sep-1978, 2791, n.spec. = 1); 18-mar-1981, 2801); (leg. M. Dimić 15-jun-1982, 1895, n.spec. = 2); Mt. Grmija (leg. L.D. Sretić 18-may-1980, 1895, n.spec. =1); Sofalija (v.) (leg. anonim. 11-jun-1980, 1896, n.spec. = 2); Priština - Uroševac: Lipljan, Mostinje ["Mostina"] (v.) (leg. G. Pasuljević 13-jan-1970, 2791, n.spec. = 7); 13-oct-1970, 2800); Prizren (leg. G. Pasuljević 22-jul-1979, 2791, n.spec. = 2); 15-aug1980, 2801); Prokuplje (leg. G. Pasuljević 13-sep-1975, 2802, n.spec. = 5); Srpska Crnja: Radojevo (v.) (leg. anonim. 11-jul-1993, 1379, n.spec. =1); (leg. G. Džukić, M. Kalezić 28-jul-1993, 721, n.spec. = 6); Stari Vlah: Sjenica, Čedovo (v.) (leg. G. Pasuljević 06-aug-1976, 2802, n.spec. = 3); Subotica (leg. anonim. 21-mar-1977, 343, n.spec. = 1); Makova Sedmica (v.), Hrastovača (leg. M. Kalezić, G. Džukić 09-apr-1998, 255, n.spec. = 1); Hrastovača, Pačarnik and Gater (leg. M. Kalezić, G. Džukić 11apr-1998, 252, n.spec. =2); Palić (v.), Mali Pijac (v.) (leg. J. Mikuška 01-may-1963, 1877, n.spec. = 
1); Tutin: Ribariće (v.) (leg. G. Pasuljević 10-jun-1978, 2801, n.spec. =10); Uroševac (leg. G. Pasuljević 12-nov-1976, 2800, n.spec. = 4); Velika Plana (leg. G. Pasuljević 27-may-1979, 2802, n.spec. = 1); Vlasina: Prisjan (v.) (leg. G. Džukić, M. Kalezić 14-jun-2006, 2313, n.spec. =1); Vlasinsko lake (leg. K. Ljubisavljević 20-aug-1998, 2044, n.spec. = 1); Zubin Potok (leg. G. Pasuljević 03-jun1978, 2802, n.spec. $=1)$.

\section{DISCOGLOSSIDAE}

\section{Bombina bombina}

S.I. (leg. anonim., s.d., 305, n.spec. = 2); (333); 531); (683); 712); (739); 954); (982); 1360); (1362); (leg. G. Džukić s.d., 1336, n.spec. = 35); Croatia Baranja (leg. J. Mikuška, 01-apr-1971, 669 2, n.spec. $=2$ ); Batsiget, Banja (leg. anonim. 08-apr-1972, 532, n.spec. $=8$ ); Tikveš (v.) (leg. J. Mikuška 26-aug-1970, 669 - 2, n.spec. = 1); Koprivnica: Peteranec (v.) (leg. anonim. 05-apr-1988, 715 , n.spec. = 1); Osijek: Kopačevski Rit (leg. G. Džukić 28-apr-1970, 710, n.spec. = 3); Sisak: Odra-Sava rivers (leg. N. Tvrtković 23-mar-1977, 1463, n.spec. = 14); Velika Gorica: Turopolje, Orle (v.) (leg. anonim. 07-apr-1988, 1432, n.spec. = 13); Peščenica (v.) (leg. N. Tvrtković 30-apr-1976, 722, n.spec. = 2); Zagreb: Dubrava (v.), Vrbovec (leg. G. Džukić 30-jun-1976, 550, n.spec. = 6); Maksimir (leg. Z. Krišković 1963, 990, n.spec. =1); Serbia Apatin (leg. anonim., 12-jun-1996, 319, n.spec. = 6); Banatska Palanka: Karaš river (leg. G. Džukić 22-mar-1970, 739, n.spec. $=1$ ); Banatski Karlovac: Seleuš (v.) - Ilandža (v.) (leg. G. Džukić, M. Kalezić 20-mar-2001, 985, n.spec. = 7); Bela Crkva: Kaluđerovo (v.) (leg. anonim. 05-apr-2012, 1320, n.spec. = 1); Leskovački Potok stream (leg. G. Džukić, M. Kalezić 28-mar-2003, 953, n.spec. = 5); 05-apr-2003, 1656); Kusić (v.) - Kaluđerovo (v.), Leskovačko lake (leg. G. Džukić, M. Kalezić, M. Marković 20may-1998, 2107, n.spec. = 21); Orešac (v.) (leg. M. Kalezić, G. Džukić, M. Marković 21-may-1998, 228, n.spec. = 4); Beograd: Borča (v.) (leg. anonim. 02-may-1994, 1163, n.spec. =1); Mali Zbeg (leg. L. Đurđević 30-oct-1994, 1049, n.spec. = 1); (leg. L. Đurđević, G. Džukić 27-mar-2005, 2196, n.spec. =1); Dobanovci (v.) (leg. G. Džukić, M. Kalezić 22-may-1993, 1445, n.spec. =4); Deliblato sands (leg. $M$. Kalezić, G. Džukić 26-jun-1999, 234, n.spec. = 3); Đurica (leg. G. Džukić, M. Kalezić 20-may-1998, 729, n.spec. = 37); (leg. M. Marković 1986, 577, n.spec. = 8); Stevanove Ravnice (leg. G. Džukić, M. Kalezić 27-feb-1999, 2103, n.spec. =6); 14-mar-1999, 2083); 18-jul-2001, 2475); Kajtasovo (v.) (leg. G. Džukić, M. Kalezić 18-mar-2001, 1186, n.spec. =1); Majur Bara (leg. G. Džukić 16-may-1976, 739, n.spec. = 12); Utrine (leg. G. Džukić, M. Kalezić 09-jul-1999, 2104, n.spec. = 21); Zubanov Salaš (leg. anonim. 17-jun-1999, 1096, n.spec. = 10); (leg. G. Džukić 22-may-1999, 968, n.spec. = 3); (leg. G. Džukić, M. Kalezić 20-apr-2000, 2086, n.spec. = 30); Horgoš: Bački Vinogradi (v.), Selevenjske Pustare (leg. anonim. 11-apr-1998, 984, n.spec. =4); Jagodina: Glogovac (v.) (leg. G. Džukić, M. Kalezić 11may-2002, 974, n.spec. =1); Ribnik (v.) (leg. G. Džukić, M. Kalezić 11-may-2002, 1113, n.spec. =2); Staro Lanište (v.), Nova Bara (leg. G. Džukić, M. Kalezić 14-may-2000, 2087, n.spec. = 50); Kikinda: Banatsko Veliko Selo (v.) (leg. G. Džukić 26-mar-2006, 1035, n.spec. = 3); Kladovo: Bajinov Rit and Iolića Rit (leg. G. Džukić, M. Kalezić 12-jun-1998, 1151, n.spec. = 27); Mala Vrbica (v.) (leg. anonim. 29-jan-1900, 983, n.spec. = 4); Kovin (leg. G. Džukić 12-jun-1970, 739, n.spec. = 2); Bavanište (v.) (leg. M. Živković 01-apr-1990, 1527, n.spec. =1); Leskovac: Vučje, Todorovce (v.), Crepanski Virovi (leg. G. Džukić, M. Kalezić 05-may-2001, 1354, n.spec. =11); Mt. Avala: Trešnja (leg. anonim. 18-may-1996, 319, n.spec. = 7); Mt. Fruška Gora: Šuljam (v.), Mutalj hamlet (leg. G. Džukić, M. Kalezić, A. Ivanović 11-may-1991, 1030, n.spec. =6); Vrdnik (v.), Vrdnička Kula (leg. G. Džukić 19-mar-1975, 739, n.spec. = 2); Mt. Vršačke Planine: Mesić (v.) (leg. anonim. 20-jun-1997, 1163, n.spec. =2); (leg. G. Džukić, M. Kalezić 18-may-1996, 1146, n.spec. = 8); 22-may-1998, 966); Široko Bilo (leg. anonim. 19-may-1991, 1852, n.spec. = 2); Negotin (leg. G. Džukić, M. Kalezić 16-may-2004, 963, n.spec. =2); Sikole (v.) (leg. anonim. 31-may-1998, 960, n.spec. = 7); (leg. G. Džukić, M. Kalezić 16-may-2004, 2013, n.spec. = 8); Novi Bečej: Slano Kopovo [“Lesino Kopovo"] (leg. G. Džukić 19-may-1976, 739, n.spec. = 6); Novi Sad: Krčedin (v.), Krčedinska Ada (ins.) (leg. G. Džukić 30-apr-1980, 1089, n.spec. = 19); Obedska Bara (leg. G. Džukić 17-apr-1970, 739, n.spec. = 3); Obrež (v.) (leg. anonim. 30-apr-1992, 1163, n.spec. =1); 10may-1996, 1163); Ravenica (leg. G. Džukić 11-may-2004, 2321, n.spec. =3); (leg. G. Džukić, M. Kalezić 
13-jun-2002, 2099, n.spec. = 38); Obrenovac: Starača (leg. G. Džukić, M. Kalezić 02-jun-1997, 1149, n.spec. =22); Kolubara river mouth (leg. G. Džukić, M. Kalezić 02-jun-1997, 736, n.spec. = 10); (965); 978); Pančevo (leg. G. Džukić, M. Kalezić 26-may-1996, 1153, n.spec. = 11); Baranda (v.) (leg. G. Džukić 25-may-1974, 739, n.spec. =1); Gradska Šuma forest (leg. G. Džukić, M. Kalezić 08-jun-1996, 1145/1, n.spec. = 10);1145/2);1145/3); Opovo (v.) (leg. M. Kalezić, A. Ivanović 11-apr-2005, 967, n.spec. = 3); Gergina Slatina (leg. G. Džukić, M. Kalezić 15-apr-2005, 733, n.spec. = 22); Pančevački Rit (leg. anonim. 19-oct-1995, 1163, n.spec. =1); Uzdinska Šuma forest (leg. G. Džukić, M. Kalezić 20-mar-2001, 2102, n.spec. = 2); Smederevo: Kulič (v.) (leg. G. Džukić 24-may-1998, 970, n.spec. = 31); Sremska Mitrovica: Leget port (leg. G. Džukić, M. Kalezić 24-apr-1998, 728, n.spec. = 26); (731); Sremski Karlovci: Bukovački Potok stream (leg. anonim. 31-jul-1979, 1142, n.spec. = 40); Karlovački Vinogradi station (leg. G. Džukić, M. Kalezić 23-apr-1995, 1144, n.spec. = 7); Srpska Crnja: Radojevo (v.) (leg. anonim. 15-jul-1995, 1163, n.spec. =3); Subotica: Makova Sedmica (v.), Hrastovača (leg. anonim. 08-mar-1998, 1163, n.spec. =1); 10-apr-1998, 1163); (leg. G. Džukić, M. Kalezić 10-apr-1998, 2105, n.spec. = 16); (leg. Moma Djurdjević 14-mar-1997, 1858, n.spec. =1); Palić (v.) (leg. G. Džukić 01-may-1963, 739, n.spec. =6); Šabac: Debrc (v.), Nikolića Bara (leg. G. Džukić 15-may-1993, 2095, n.spec. = 1); Orid (v.) (leg. G. Džukić, M. Kalezić 27-apr-1998, 732, n.spec. = 44); Provo (v.), Orlača, Ključ (leg. R. Ajtić 01-jun-2006, 2129, n.spec. =6); Trbušac (v.) (leg. G. Džukić, M. Kalezić 27-apr-1998, 2078, n.spec. = 12); Valjevo (leg. anonim. 26-apr-1998, 1163, n.spec. = 2); Velika Plana: Žabari (v.), Šljunkara (leg. anonim. 24-may-1998, 973, n.spec. = 6); Vrnjačka Banja: Ugljarevo (v.) (leg. N. Labus 17-may-2001, 737, n.spec. = 5); Vršac: Vatin (v.) (leg. G. Džukić, M. Kalezić 07-may-2004, 1637, n.spec. = 17); Zaječar: Trnavac (v.), Majino Okno (leg. G. Džukić, M. Kalezić, M. Slijepčević, A. Urošević 23-may-2012, 1317, n.spec. = 2); Zrenjanin: Ečka (v.) (leg. G. Džukić 19-oct-1970, 739, n.spec. = 1); Melenci (v.), Ostrovo (leg. G. Džukić 04-oct-1996, 1396, n.spec. =67); Samoš (v.) (leg. G. Džukić, M. Kalezić 03-jun-2000, 1150, n.spec. =24).

\section{Bombina bombina / variegata}

Serbia Mt. Fruška Gora (leg. G. Džukić, M. Kalezić, 10-may-1996, 1126, n.spec. = 21); Andrevlje (leg. G. Džukić, M. Kalezić 10-may-1996, 1161, n.spec. = 33); Osovlje (leg. G. Džukić, M. Kalezić 10-may-1996, 1157, n.spec. = 25); Šuljam (v.), Mutalj hamlet (leg. G. Džukić, M. Kalezić 11may-1991, 1456, n.spec. $=14)$.

\section{Bombina variegata}

S.I. (leg. anonim., s.d., 304, n.spec. = 2); (321); 333); (357); (531); 546); (549); 951); (958); 981); (992); 997); (1007); 1260); (1309); 1346); (1373); 1433); (1435); 1864); (leg. G. Džukić s.d., 990, n.spec. =6); (996); 1270); (1272); (1304); Bosnia and Herzegovina Čajniče (leg. anonim., 21-sep1991, 1501, n.spec. = 5); Derventa: Vlasenica, Tišća river wellhead (leg. G. Džukić 23-jul-1970, 1294, n.spec. = 6); Doboj: Tešanj, Raduša (v.) (leg. S. Lelo 25-may-2003, 1286, n.spec. = 19); Gacko (leg. anonim. 02-may-2004, 734, n.spec. = 1); Lukovica (v.) (leg. G. Džukić 03-aug-1970, 1294, n.spec. = 3); Kupres (leg. G. Džukić, M. Kalezić 17-may-1983, 2748, n.spec. =1); Bukavička Previja, lake (leg. G. Džukić 05-jun-1985, 2522, n.spec. =1); Kurića Poljane (leg. G. Džukić 04-jun1985, 2640, n.spec. = 2); Mostar: Bunica spring (leg. anonim. 03-aug-1979, 2098, n.spec. = 30); Drežnica (v.) (leg. E. Memišević 09-may-2004, 1290, n.spec. = 2); Mt. Bjelašnica (leg. G. Džukić 04aug-1970, 1294, n.spec. = 1); Mt. Čvrsnica (leg. anonim. 20-jun-1971, 710, n.spec. = 1); Mt. Igman (leg. G. Džukić 01-aug-1974, 1281, n.spec. =12); Mt. Maglić: Perućica (leg. G. Džukić, M. Kalezić 24aug-2002, 1297, n.spec. = 5); Mt. Ozren: Crni Vrh (leg. G. Džukić 24-jul-1970, 1294, n.spec. = 2); Mt. Romanija: Sokolac, Pediše (v.) (leg. S. Lelo 31-jul-2003, 738, n.spec. =10); Mt. Volujak (leg. S. Lelo s.d., 735, n.spec. = 4); Sutjeska river (leg. S. Lelo s.d., 745, n.spec. = 1); Mt. Vranica: Prokoško lake (leg. E. Memišević 19-jul-2003, 727, n.spec. = 3); (leg. S. Lelo 20-jul-2003, 1330, n.spec. = 16); 22-jul-2003, 1404); Mt. Zvijezda (leg. S. Lelo 16-jun-2003, 1331, n.spec. = 15); Posušje: Poklečani (v.) (leg. anonim. 21-jun-1971, 710, n.spec. = 1); Sarajevo: Kijevo (v.), Železnica river (leg. G. Džukić 12-may-1983, 694/1, n.spec. =9); Tuzla: Moluhe (v.) (leg. anonim. 25-aug-2003, 955, n.spec. = 7); (leg. S. Lelo 24-25-may-2003, 2160, n.spec. = 28); Bulgaria Mt. Zapadna Stara Planina: Lokorsko (v.) (leg. Lj. Tomović, B. Naumov, 10-oct-2004, 2009, n.spec. = 
6); Croatia Donji Lapac: Lapačka Korita (leg. G. Džukić, 21-may-1987, 1482, n.spec. = 1); Gorski Kotar: Crni Lug (v.) (leg. anonim. 14-jul-1964, 1156, n.spec. = 3); Istra: Pazin (leg. G. Džukić 1976, 1399, n.spec. = 4); Grbaljica (leg. G. Džukić 23-jun-1976, 1268, n.spec. = 27); Mt. Medvednica (leg. D. Pelić, G. Džukić 01-apr-1975, 693, n.spec. = 5); Mt. Papuk (leg. G. Džukić 1976, 1399, n.spec. = 2); Split (leg. G. Džukić 01-may-1976, 1375, n.spec. =4); Varaždin: Donja Voća (v.) (leg. B. Jalžić 18-jul-1976, 1343, n.spec. = 1); Velika Gorica: Vukomeričke Gorice, Dragonožec (v.), Trpuci (leg. N. Tvrtković 18-apr-1976, 707, n.spec. =11); Zagreb: Gornja Kustošija (v.) (leg. G. Džukić 23-apr-1981, 1850, n.spec. = 1); Maksimir (leg. D. Pelić 10-apr-1976, 724, n.spec. =1); Macedonia Berovo: Berovsko lake, Ratevska river (leg. G. Džukić, 26-may-1970, 710, n.spec. = 4); Mt. Babuna: Stepanci (v.), Sv. Dimitrije monastery (leg. G. Džukić 27-jun-1998, 2674, n.spec. =2); Mt. Galičica (leg. G. Džukić 19-sep-1978, 1287, n.spec. = 5); Mt. Korab: Ćos Alija (leg. G. Džukić, M. Vehbi 18-sep-1996, 2280, n.spec. =1); Kobilino Polje (leg. G. Džukić 16-sep-1996, 2283, n.spec. = 2); Mt. Osogovske Planine: Ponikva (leg. G. Džukić, S. Petkovski 23-jun-1995, 215, n.spec. = 3); Mt. Pelister: Magarevska river (leg. G. Džukić 25-jul-1979, 1303, n.spec. = 2); Mt. Skopska Crna Gora: Vlaška river, Pasji Potok stream (leg. G. Pasuljević 03-jun-1975, 356, n.spec. = 2); Zdravačka river (leg. G. Pasuljević 08-aug-1979, 1164, n.spec. = 4); Probištip: Lesnovo (v.) (leg. M. Kalezić 06-may-1989, 980, n.spec. =1); Struga: Belčišta (v.) (leg. G. Džukić, M. Kalezić 06-may-1997, 1377, n.spec. = 5); Podgorci (v.) (leg. anonim. 22-sep-1996, 1298, n.spec. = 21); (leg. G. Džukić, M. Kalezić 22-sep-1996, 1351, n.spec. =24); Strumica: Suvi Laki (leg. G. Džukić 29-may-1970, 710, n.spec. = 2); Montenegro Berane [“Ivangrad”] (leg. M. Račić, 06-sep-1979, 1164, n.spec. = 2); 16-sep-1979, 1164); (leg. V. Radičević 09-sep-1979, 1164, n.spec. = 2); (leg. V. Smiljanić 05-may-1980, 1164, n.spec. = 10); Budimlje (v.) (leg. Z. Boševska, D. Todorović 18-jul-1976, 356, n.spec. = 4); pond (leg. D. Rajković, G. Pasuljević 30-may-1975, 356, n.spec. =13); Trpeška Rijeka stream (leg. I. Skenderović 20-aug-1987, 356, n.spec. = 5); Cetinje: Bjeloši (v.) (leg. G. Džukić, M. Kalezić 27-may-1996, 748, n.spec. = 16); Država (leg. G. Džukić, M. Kalezić 27-may-1996, 1376, n.spec. = 15); Lašor puddle (leg. G. Džukić, M. Kalezić 30-may-1990, 720, n.spec. =1); Ćeklići, Kućišta (v.), Crno Korito (leg. M. Kalezić, G. Džukić, M. Marković 20-jun-2004, 2440, n.spec. = 3); Ublica (leg. M. Kalezić, G. Džukić, M. Marković 20-jun-2004, 2523, n.spec. =1); Cetinje-Grahovo: Ržani Do (v.), Cape (leg. M. Kalezić, G. Džukić, M. Marković 20-jun-2004, 952, n.spec. =1); Zlovrćan (leg. M. Kalezić, G. Džukić, M. Marković 20-jun-2004, 746, n.spec. =1); Danilovgrad: Cicmarovica (leg. G. Džukić, M. Kalezić 25-apr-2002, 979, n.spec. =2); Čevo (v.), Čevske Ubli (v.) (leg. G. Džukić, M. Kalezić 19-jun-2004, 988, n.spec. = 2); Donji Zagarač (v.) (leg. G. Džukić, M. Kalezić 26-apr-2002, 742, n.spec. = 4); Milojevići (v.), Mrainjac (leg. G. Džukić, M. Kalezić 06-jun-2005, 2322, n.spec. =1); Mt. Garač (leg. M. Kalezić, G. Džukić, M. Marković 29-may-2003, 2289, n.spec. =1); Erakovice (leg. M. Kalezić, G. Džukić, M. Marković, M. Ćirović, D. Šipčić 29-may-2003, 2281, n.spec. =1); Vodine (leg. G. Džukić 25-apr-2002, 1282, n.spec. = 5); Zagarač (v.) (leg. anonim. 24-may-1996, 1300, n.spec. = 12); Miogos (v.) (leg. G. Džukić, M. Kalezić 22-aug-2001, 1283, n.spec. =7); Grahovo: Donji Izvor (v.) (leg. M. Kalezić, G. Džukić, M. Marković 20-jun-2004, 2512, n.spec. =1); Razmuđe (v.), Razmućski Dol, Razmućska Kamenica (leg. G. Džukić, M. Kalezić 20-jun-2004, 2301, n.spec. =1); (leg. M. Kalezić, G. Džukić, M. Marković 20-jun-2004, 1293, n.spec. =6); Rokoči (v.), Meka Lokva (leg. M. Kalezić, G. Džukić, M. Marković 20-jun-2004, 2291, n.spec. = 2); Kolašin: Morača monastery (leg. L. Vlahović 29nov-1978, 356, n.spec. = 12); Kostiće (v.) (leg. M. Vesilović 01-aug-1982, 1895, n.spec. =1); (leg. M. Vesković 01-aug-1982, 357, n.spec. =4); Kotor: Škudra (leg. G. Džukić 14-jul-1969, 710, n.spec. =1); 26-may-1996, 991); Mt. Bioč: Stabna (v.), Stubica (v.), Brštevac (leg. G. Džukić, M. Kalezić 06-jun2008, 2231, n.spec. =1); Mt. Bjelasica: Biogradska Gora (leg. Lj. Tomović, R. Ajtić 22-jul-2000, 1830, n.spec. = 4); Biogradsko lake (leg. G. Džukić, J. Crnobrnja-lsailović 07-jul-1996, 1378, n.spec. = 19); (leg. G. Pasuljević 16-jun-1970, 356, n.spec. =1); Goleš Katun challet, road to (leg. G. Džukić, J. Crnobrnja-Isailović, I. Aleksić 24-jul-1997, 2664, n.spec. =9); Lalevića Dolovi, Nicovića Lokva puddle (leg. G. Džukić, J. Crnobrnja-Isailović, I. Aleksić 25-jul-1997, 311, n.spec. =2); Sezerine (leg. G. Džukić, J. Crnobrnja-Isailović, I. Aleksić 29-jul-1997, 316, n.spec. =1); Voleš, road to (leg. G. Džukić, J. CrnobrnjaIsailović, I. Aleksić 24-jul-1997, 309, n.spec. = 1); Mt. Durmitor (leg. anonim. s.d., 682, n.spec. $=1$ ); (1310); 1314); (1400); 1630); Žabljak, Radovan Luka (leg. anonim. 14-sep-1990, 1448, n.spec. =1); Mt. Lovćen: Ivanova Korita (leg. G. Džukić 28-jul-1988, 719, n.spec. = 45); Mt. Lukavica: Kapetanovo lake (leg. G. Džukić, M. Kalezić 23-aug-2002, 2521, n.spec. =1); Mt. Rumija: Stari Bar, Virosovica (leg. anonim. 28-aug-1997, 1288, n.spec. = 8); Sutorman (v.), Lera (leg. G. Džukić, M. Kalezić 02-jun-1990, 1523, n.spec. = 7); Ublić (leg. S. Vuletić 01-may-1992, 652, n.spec. = 2); Mt. 
Žijovo: Bukumirsko lake (leg. G. Džukić, M. Kalezić 28-may-2003, 961, n.spec. = 17); Mutno lake (leg. G. Džukić, M. Kalezić 21-aug-2001, 2328, n.spec. =1); Širokar, Zokanj (leg. anonim. 18-sep-1990, 2742, n.spec. =1); Mts Prokletije: Gusinje, Grbaja (leg. S. Đorđević 03-jul-2004, 1832, n.spec. =1); Nikšić: Ponikvica (v.) (leg. G. Džukić, M. Kalezić 10-jun-1997, 998, n.spec. =17); middle (leg. G. Džukić, M. Kalezić 10-jun-1997, 1353, n.spec. = 17); Župa, Morakovo (v.), Zabran Kralja Nikole (leg. G. Džukić, M. Kalezić 23-aug-2001, 743, n.spec. =2); Plav: Ali-Pašin Bunar (leg. G. Pasuljević 22-aug1980, 1164, n.spec. =2); Brezojevica (v.) (leg. G. Pasuljević 15-aug-1974, 356, n.spec. =2); Pljevlja (leg. R. Malidžan 25-nov-1976, 356, n.spec. = 1); Podgorica: Cijevna river (leg. K. Ljubisavljević 03sep-1999, 2106, n.spec. =1); Komani, Vojova Kamenica (leg. G. Džukić, M. Kalezić 26-apr-2002, 740, n.spec. = 2); Medun (v.) (leg. anonim. 19-sep-1991, 1488, n.spec. =12); Cisterna (leg. G. Džukić, M. Kalezić, R. Ćirović 21-aug-2001, 1296, n.spec. = 13); Progonovići (v.), Releza (v.), Razvađa puddle (leg. G. Džukić, M. Kalezić 24-apr-2002, 749, n.spec. =1); 28-aug-2001, 977); Stabanovi Gornji (leg. anonim. 22-aug-2001, 1299, n.spec. =6); Seoca (v.), Krnjac Do (leg. M. Kalezić, G. Džukić, M. Marković 21-jun-2004, 744, n.spec. = 2); Rijeka Crnojevića: Jankovića Krš (v.), Pištet (leg. anonim. 04-jul1992, 1520, n.spec. = 12); Rožaj: Ibar river (leg. A. Ago, G. Pasuljević 01-jul-1974, 356, n.spec. = 5); Skadarsko lake: Krajina, Gornja Briska (v.) (leg. anonim. 21-apr-1991, 1385, n.spec. =1); Livari (v.) (leg. anonim. s.d., 1457, n.spec. =2); (leg. G. Džukić, M. Kalezić 30-may-1996, 1349, n.spec. = 20); Šavnik: Pošćensko lake (leg. G. Džukić, J. Crnobrnja-Isailović, I. Aleksić 30-jul-1997, 2682, n.spec. = 1); Tara river canyon: Tepca (v.) (leg. M. Langurov, Lj. Tomović 01-jul-1998, 1155, n.spec. = 5); Vilusi: Banjani, Podljut (v.), Kadaruša (leg. G. Džukić, M. Kalezić 10-jun-2005, 2306, n.spec. =1); Kešelj Gradina (v.) (leg. G. Džukić, M. Marković, M. Kalezić 26-jun-2004, 2017, n.spec. =99); Serbia (leg. anonim., s.d., 972, n.spec. = 59); (1872); Aleksandrovac: Vrbnica (v.) (leg. G. Džukić 03-jun1977, 972, n.spec. = 2); Beograd: Barajevo (v.) (leg. anonim. 21-jun-1998, 1118, n.spec. $=5$ ); Blace (leg. N. Miletić 15-sep-1976, 356, n.spec. = 1); Bor (leg. anonim. 20-may-1979, 1164, n.spec. =2); Bosilegrad (leg. G. Džukić, M. Kalezić 20-jun-1996, 1127, n.spec. = 31); Donja Ljubata (v.) (leg. anonim. 25-sep-2005, 1823, n.spec. = 13); Mlekominci (v.) (leg. J. Crnobrnja-Isailović, I. Aleksić 03-jun1998, 2090, n.spec. = 22); Zli Dol (v.) (leg. G. Džukić, M. Kalezić 16-jun-2006, 1116, n.spec. = 5); (leg. M. Kalezić 23-apr-2014, 2385, n.spec. =1); Crna Trava: Biljine Bare (leg. G. Džukić, M. Kalezić 23-jun2007, 2186, n.spec. = 6); Ćićevac: Braljina (v.), Veliki Potok (leg. anonim. 01-aug-1979, 1356, n.spec. = 35); Dimitrovgrad: Smilovci (v.) (leg. G. Džukić, M. Kalezić 15-may-2004, 1641, n.spec. = 3); Đakovica (leg. S. Šalja 23-jul-1980, 1164, n.spec. = 5); Babaj Boks (v.) (leg. G. Pasuljević, G. Džukić, B. Milošević 18-jul-1980, 1164, n.spec. =2); Gnjilane: Donji Livoč (v.) (leg. G. Pasuljević 10oct-1969, 356, n.spec. = 2); Kosovska Kamenica, Ograđe (leg. B. Milošević 28-aug-1978, 356, n.spec. = 1); Sveti Jovan (leg. B. Milošević 18-aug-1977, 356, n.spec. = 7); Grdelička gorge: Migulovac (v.) (leg. G. Džukić, M. Kalezić 18-apr-2007, 1640, n.spec. = 5); Kladovo: Manastirski Gaj (leg. anonim. 15-jul-1998, 747, n.spec. = 19); (leg. G. Džukić, M. Kalezić 15-jul-1998, 1115, n.spec. $=$ 7); Zamna river (leg. G. Džukić, M. Kalezić 28-sep-2005, 2277, n.spec. =1); Zamna river gorge (leg. G. Džukić, M. Kalezić 22-jun-2003, 971, n.spec. =3); Kladovo-Negotin: Vratna (v.) (leg. G. Džukić 30apr-1972, 1270, n.spec. = 5); Klina: Beli Drim river (leg. B. Koprivica 17-jun-1977, 356, n.spec. = 3); Knjaževac: Žukovska river (leg. anonim. 15-jun-1976, 1007, n.spec. = 6); Kosovo (leg. G. Pasuljević s.d., 356, n.spec. = 20); Kosovska Mitrovica: Banjska (v.) (leg. D. Todorović 07-jun-1977, 356, n.spec. = 23); 03-jun-1981, 1164); 28-oct-1977, 356); Grižane (v.) (leg. D. Todorović 18-apr-1978, 356, n.spec. $=14$ ); Jankov Potok stream (leg. D. Todorović 14-nov-1977, 356, n.spec. $=2$ ); Ješevik (leg. D. Todorović 08-apr-1978, 356, n.spec. = 6); Junake (v.) (leg. D. Todorović 08-jun-1978, 356, n.spec. = 2); Lovac (v.) (leg. D. Todorović 02-may-1978, 356, n.spec. =1); Rudnik (leg. D. Todorović 11apr-1978, 356, n.spec. $=24$ ); Vakač (leg. D. Todorović 04-may-1978, 356, n.spec. =4); Banjska river (leg. D. Todorović 01-apr-1978, 356, n.spec. = 5); Leposavić (leg. anonim. 18-jun-1973, 356, n.spec. = 5); Dren (v.) (leg. G. Pasuljević 24-may-1975, 356, n.spec. = 1); Zabrđski Potok stream (leg. G. Pasuljević 24-may-1975, 356, n.spec. = 1); Srbovac (v.) (leg. D. Todorović 26-may-1979, 1164, n.spec. = 1); Zvečan (v.), Rudine (v.) (leg. Ž. Spasić 20-sep-2003, 1147/1, n.spec. = 17); 1147/2); Kraljevo (leg. M. Lazović 05-may-1983, 357, n.spec. = 3); Žiča (v.) (leg. S. Grujić, S. Labus, Z. Stojanović 18-aug1982, 357, n.spec. =9); Kruševac (leg. D. Rajković 10-jul-1976, 356, n.spec. =2); Ribarska Banja spa (leg. anonim. 19-jul-1997, 1163, n.spec. = 3); Kučevo: Blagojev Kamen (v.) (leg. G. Džukić 08jun-1972, 1270, n.spec. = 3); Kuršumlija: Bela Crkva (v.) (leg. anonim. 20-may-1998, 976, n.spec. =10); Prolom Banja spa (leg. G. Pasuljević 11-jun-1979, 1164, n.spec. $=2$ ); Majdanpek: Debeli Lug (v.), Todorova river (leg. G. Džukić, M. Kalezić 27-apr-2009, 1065, n.spec. =3); (2144); Železnik 
(v.) - Debeli Lug (v.) (leg. V. Kalafatić 26-may-1980, 1633, n.spec. =1); Medveđa: Jablanica, Buzuluk (v.) (leg. G. Džukić 17-may-1977, 972, n.spec. =1); Mladenovac: Sopot (v.), Ropočevo (v.) (leg. G. Džukić, M. Kalezić 12-jul-1999, 1125, n.spec. = 8); 25-jul-1999, 1125); Mt. Beljanica (leg. anonim. 17-jul-2004, 1831, n.spec. =1); Rečke (leg. T. Vukov 12-jul-1998, 1121, n.spec. =16); Mt. Besna Kobila: Kriva Feja (v.), lake (leg. G. Džukić, M. Kalezić 17-jun-2006, 1137, n.spec. =15); (1154); Mt. Fruška Gora (leg. anonim. 12-may-1998, 317, n.spec. =10); Andrevlje (leg. G. Džukić 11-may-1975, 1270, n.spec. = 2); 12-may-1975, 1270); (leg. G. Džukić, M. Kalezić 08-jun-1996, 1112, n.spec. = 6); Brankovac (leg. N. Tomašević, T. Vukov 24-jul-2005, 2019, n.spec. =10); Greben (leg. G. Džukić, M. Kalezić 08-jun-1996, 1111/1, n.spec. = 8); 1111/2); 1111/3); Osovlje (leg. G. Džukić 17-may1975, 1270, n.spec. =1); (leg. G. Džukić, M. Kalezić 25-may-1998, 2085, n.spec. = 18); Šuljam (v.) (leg. anonim. 11-may-1991, 741, n.spec. =4); Zmajevac (leg. G. Džukić 19-aug-1975, 725, n.spec. =1); Mt. Golija (leg. G. Džukić 18-sep-1967, 1294, n.spec. =2); Pridvorica (v.) (leg. G. Džukić, M. Kalezić 14jun-2001, 1114, n.spec. = 5); Mt. Jadovnik (leg. J. Crnobrnja-Isailović 27-aug-1997, 2006, n.spec. = 7); Mt. Jagodnja: Mačkov Kamen (leg. G. Džukić, M. Kalezić 01-jun-1997, 1134, n.spec. = 13); Mt. Javor: Bukovik (v.) (leg. G. Džukić, M. Kalezić 27-aug-2001, 1110, n.spec. =2); (leg. S. Marinković 17jul-1993, 1863, n.spec. = 2); Kušići (v.) (leg. G. Džukić, M. Kalezić 30-jul-1996, 1123, n.spec. = 20); Vasilin Vrh (leg. M. Paunović 16-may-1994, 2132, n.spec. = 2); Vasiljev Vrh (leg. anonim. 29-jul1996, 1124/2, n.spec. =12); (leg. G. Džukić, M. Kalezić 29-jul-1996, 1263, n.spec. =12); 1124/1); Mt. Južni Kučaj (leg. G. Džukić, M. Kalezić 03-jul-2004, 2024, n.spec. = 3); Mt. Kopaonik: Jošanička Banja spa (leg. O. Glišić 29-apr-1986, 958, n.spec. =4); Mt. Koritnik (leg. M. Hajredini 15-sep-1981, 357, n.spec. = 1); (1895); Mt. Kosmaj (leg. G. Džukić, M. Kalezić 25-jul-1999, 2094, n.spec. = 6); Ropočevo (v.) (leg. G. Džukić, M. Kalezić 25-jul-1999, 956, n.spec. = 11); (2336); Mt. Kukavica: Đokini Virovi, Gneždo and Jasiško Zavište (leg. G. Džukić, M. Kalezić 16-jun-2001, 1136, n.spec. = 23); Mt. Miroč: Brza Palanka (v.), Stara Brza (v.), Balta Prun (leg. G. Džukić, M. Kalezić 15-jul1998, 1001, n.spec. = 14); (1139); Potrovo (v.), Majdan (leg. G. Džukić, M. Kalezić 25-apr-2009, 1063, n.spec. =1); Mt. Ostrozub: Ruplje (v.) (leg. G. Džukić, M. Kalezić 12-sep-2006, 1140, n.spec. = 2); (2290); Selišta (leg. G. Džukić, M. Kalezić 20-apr-2007, 2185, n.spec. =1); Mt. Paštrik (leg. G. Pasuljević, G. Džukić, B. Milošević 19-jul-1980, 1164, n.spec. =2); Mt. Radan (leg. G. Džukić 21-may1977, 972, n.spec. = 4); Mt. Rtanj: Draženovac (leg. anonim. 20-aug-1979, 1128, n.spec. $=20$ ); Javor (leg. D. Mišić 19-may-2004, 1638, n.spec. = 5); Mt. Stara Planina: Brlog (v.) (leg. G. Džukić, M. Kalezić 04-sep-2004, 2018, n.spec. = 1); Dojkinci (v.) (leg. G. Džukić, M. Kalezić 27-jul-1998, 1129, n.spec. = 19); Široke Luke (leg. N. Labus 28-may-2002, 1000, n.spec. =4); Toplodolska river (leg. P. Jakšić 14-jul-1991, 1222, n.spec. = 9); Mt. Starac (leg. G. Džukić, M. Kalezić 19-jul-2004, 969, n.spec. = 5); Mt. Tara: Batura (leg. G. Džukić, M. Kalezić 30-aug-2002, 2536, n.spec. =1); Beli Rzav lake (leg. anonim. 12-jun-2001, 2470, n.spec. = 8); (leg. G. Džukić, M. Kalezić 12-jun-2001, 1133, n.spec. = 8); Bulibanovac (v.) (leg. G. Džukić, M. Kalezić 30-jun-2008, 1220, n.spec. $=22$ ); Crni Vrh, Kapetanova Voda (leg. anonim. 22-may-1972, 2470, n.spec. =1); (leg. G. Džukić 22-may-1972, 1270, n.spec. =1); Jagoštica (v.), Čairi, Veliki Kraj, Zvijezda (leg. anonim. 23-may-1972, 2470, n.spec. = 4); Galine hamlet (leg. anonim. 21-may-1972, 2470, n.spec. = 3); Rastište (v.), Požar hamlet (leg. anonim. 21may-1972, 2470, n.spec. =1); Sokolina (leg. anonim. 15-may-1972, 2470, n.spec. =16); (leg. G. Džukić s.d., 1270, n.spec. = 16); Šljivovica (v.) (leg. anonim. 09-jun-1969, 2470, n.spec. = 2); Zaovine (v.), Beli Rzav lake (leg. anonim. 20-may-1972, 2470, n.spec. = 4); (leg. G. Džukić 20-may-1972, 1270, n.spec. =4); Mt. Vršačke Planine: Mesić (v.) (leg. anonim. 02-aug-1991, 1860, n.spec. = 2); Mt. Zlatar (leg. J. Crnobrnja-Isailović 07-jul-1996, 1392, n.spec. = 13); Aljinovići (v.), Goveđak (leg. anonim. 11-sep-1991, 1845, n.spec. = 19); Mt. Zvijezda: Jastočica (v.), Čairi, Veliki Kraj (leg. G. Džukić 23-may-1972, 1270, n.spec. = 5); Mts Prokletije (leg. V. Radičević s.d., 1261, n.spec. = 8); Erenik stream (leg. G. Džukić, M. Kalezić 15-jun-1997, 1130, n.spec. =17); Mt. Mokra Gora, Ponor (leg. G. Džukić 12-jun-1977, 972, n.spec. = 3); Mts Šar-Planina (leg. G. Pasuljević 05-jun-1971, 356, n.spec. = 5); Štrpce (leg. P. Stamenković 15-jun-1977, 356, n.spec. = 8); Nova Varoš: Šupljica (v.), Neškoviće (v.) (leg. G. Džukić 11-aug-1982, 1270, n.spec. =11); Orahovac: Miruša river (leg. G. Pasuljević, B. Milošević 29-jun-1980, 357, n.spec. = 3); (1164); Paraćin: Sisevac (v.), Grza river (leg. anonim. 14-may-1977, 1007, n.spec. =1); Paraćin - Boljevac: Krivi Vir (v.), Izvor (v.), Garska Česma (leg. G. Džukić, M. Kalezić 16-may-2000, 1119, n.spec. =12); Peć (leg. Lj. Marković 21-sep-1976, 356, n.spec. = 2); (leg. S. Bašanović 18-sep-1976, 356, n.spec. = 3); Dečane (leg. G. Džukić 05-jun1977, 972, n.spec. = 9); Visoki Dečani monastery (leg. G. Pasuljević, G. Džukić, B. Milošević 17-jul-1980, 1164, n.spec. = 2); Đurakovac (v.), Osojane (v.) (leg. D. Lakušić 05-oct-1978, 356, n.spec. = 2); 
Istok (v.) (leg. G. Džukić 05-jun-1977, 972, n.spec. = 2); (leg. G. Pasuljević 25-nov-1970, 356, n.spec. = 3); Karagač (leg. anonim. 31-jul-1995, 1163, n.spec. = 1); Lugovo (v.) (leg. G. Džukić 06-jun-1977, 972, n.spec. = 3); Ljubovo (v.) (leg. H. Babačić 01-sep-1978, 356, n.spec. = 2); Novo Selo (v.), Radovče (leg. anonim. 09-jun-1997, 1163, n.spec. =2); Vitomirica (v.) (leg. H. Babačić 15-jul-1978, 356, n.spec. = 3); Zatra (v.) (leg. anonim. 13-sep-1978, 356, n.spec. = 4); Zobijak (leg. H. Babačić 08sep-1978, 356, n.spec. = 3); Pešter Plateau: Delimeđe (v.), Las Sadov (leg. G. Džukić, M. Kalezić 29-apr-2010, 2673, n.spec. = 9); Mt. Jarut (leg. anonim. 29-apr-2010, 2207, n.spec. = 1); Pirot: Cerova (v.) - Temska (v.) (leg. G. Džukić, M. Kalezić 25-sep-2008, 1057, n.spec. =1); Kamik (v.) (leg. G. Džukić, M. Kalezić 14-jun-2006, 2323, n.spec. =6); Zvonce (v.) (leg. G. Džukić, M. Kalezić 02jun-1998, 1135, n.spec. =22); Požarevac: Zaova monastery (leg. G. Džukić, M. Kalezić 23-may1998, 1132, n.spec. = 14); Preševo (leg. anonim. 05-jun-1998, 1163, n.spec. = 1); Borovac (v.) (leg. G. Džukić, M. Kalezić 21-may-2008, 1247, n.spec. = 21); Previja (leg. G. Džukić 08-jun-1977, 972, n.spec. = 3); Priboj (leg. E. Klajić 10-jun-1982, 357, n.spec. =2); Priština (leg. anonim. s.d., 356, n.spec. =3); Gračaničko lake (leg. B. Batvić 10-may-1977, 356, n.spec. = 3); Kišnica (v.) (leg. B. Bojčetić 18may-1984, 357, n.spec. = 1); Kosovo Polje (leg. D. Čukić 10-aug-1980, 1895, n.spec. = 2); Mt. Grmija (leg. O. Papović 19-jun-1981, 1895, n.spec. = 2); Grušja (leg. O. Papović 19-jun-1981, 356, n.spec. = 1); Obilić, vicinity (leg. G. Pasuljević 01-apr-1967, 1262, n.spec. =1); Prizren: Gručare (v.) (leg. G. Pasuljević 30-jun-1979, 1164, n.spec. =2); Prohor Pčinjski monastery: Mt. Starac (leg. G. Džukić, M. Kalezić 21-aug-1994, 994, n.spec. = 15); Prokuplje (leg. Z. Đorđević 10-sep-1978, 356, n.spec. =9); Konjuša (v.) (leg. D. Gagulić? 13-sep-1976, 356, n.spec. = 1); Raška: Aljinovići (v.) (leg. S. Marinković 07-aug-1991, 1307, n.spec. = 1); Jošanica (leg. anonim. 12-may-1977, 356, n.spec. = 1); Sićevačka gorge: Banjica (leg. G. Džukić, M. Kalezić 08-jul-1997, 1143, n.spec. = 23); Ostrovica (v.) (leg. anonim. 05-jul-1997, 986, n.spec. =6); Stari Vlah: Sjenica (leg. G. Džukić, M. Kalezić 27-apr-2010, 2200, n.spec. =1); 28-aug-1997, 1002); Čedovo (v.) (leg. D. Mijajlović 10-jun-1976, 356, n.spec. = 4); Raždaginja (v.) (leg. H. Mešić, G. Pasuljević 20-aug-1975, 356, n.spec. = 2); Surdulica: Jelašnica river gorge (leg. G. Džukić 12-jul-1977, 972, n.spec. = 2); Takovo: Savinac (v.) - Drenova river (leg. D. Kataranovski 03-aug-1998, 1514, n.spec. = 8); Trstenik: Donja Crnišava (v.), Parlozi (leg. K. Ljubisavljević 26-jul-1997, 1120, n.spec. = 10); Tutin: Ribariće (v.) (leg. B. Prelević 10-jun-1978, 356, n.spec. = 8); Uroševac (leg. R. Savić 03-jun-1979, 1164, n.spec. = 5); (leg. S. Novičević; D. Mijajlović 15-jun-1976, 356, n.spec. =7); Nerodimlje (v.) (leg. D. Marković 07-sep-1980, 1164, n.spec. = 4); Vitina (leg. G. Pasuljević 24-may-1972, 356, n.spec. =1); Valjevo: Bukovac (v.), Oreovac (leg. M. Kalezić, G. Džukić 02-sep-2004, 2114, n.spec. = 6); Đurđevac (v.) (leg. G. Džukić, M. Kalezić 16-apr-2005, 1152, n.spec. = 3); Gola Glava (v.) (leg. G. Džukić, M. Kalezić 26-apr-1998, 2080, n.spec. = 9); Gradac river gorge (leg. G. Džukić 21-jun-1983, 1321, n.spec. = 19); Joševa (v.) (leg. G. Džukić, M. Kalezić 17-apr-2005, 1159, n.spec. = 1); Kamenica (v.) (leg. M. Kalezić 29-aug-2004, 2015, n.spec. = 8); Gaj (leg. M. Kalezić 04-aug-2005, 2025, n.spec. = 5); Koceljeva, Subotica (v.), Đaković (leg. anonim. 25-apr-1998, 1138, n.spec. = 27); Petnica (v.) (leg. anonim. 31-may-1997, 1163, n.spec. =2); Vlasina: Gracka river (leg. G. Džukić, M. Kalezić 13-sep-2006, 2295, n.spec. = 2); Jerma river (leg. G. Džukić, M. Kalezić 25-jun-1998, 1117, n.spec. = 5); Klisura (v.) (leg. G. Džukić, M. Kalezić 16-jun-2006, 2308, n.spec. = 1); 21-sep-2007, 1255); Prisjan (v.) (leg. G. Džukić, M. Kalezić 14-jun-2006, 2313, n.spec. = 1); Vranje: Mt. Krstilovica, Devotin, lake (leg. M. Kalezić, G. Džukić, M. Marković 24-sep-2005, 2558, n.spec. = 4); Vučitrn: Samodraža (v.) (leg. anonim. 04-jul-1969, 1007, n.spec. = 1); (leg. R. Malidžan, Z. Boševska, N. Miletić, S. Jeftić, D. Mijajlović 27-jun-1976, 356, n.spec. =12); Zlot (leg. J. Crnobrnja-Isailović, I. Aleksić 17-jun-1996, 1335, n.spec. = 4); Lazareva river canyon, Zlotska cave (leg. anonim. 14-may-1970, 1007, n.spec. = 16); Žagubica: Lisaja (v.) (leg. G. Džukić, M. Kalezić 28-may-1998, 1122, n.spec. = 24); Suvi Do (v.) (leg. G. Džukić, M. Kalezić 29-jun-1998, 1131, n.spec. = 15); Slovenia Ajdovščina: Štanjel (v.), Kobjeglava (leg. B. Kryštufek, 07-may-1986, 714, n.spec. =1); Ljubljana: Ig (v.) (leg. G. Džukić 01-apr-1979, 2091, n.spec. =9); Novo Mesto: Mirna (v.), Lavrica, Smrečje, Čed stream (leg. J. Gregori, B. Horvat 10-jun-1986, 714, n.spec. =1); Zabrđe (leg. B. Kryštufek, B. Horvat 23apr-1986, 714, n.spec. = 3); Piran: Dragonja river, Inpinjevca (leg. B. Kryštufek 01-may-1986, 726, n.spec. = 2); Postojna: Mt. Slavinček, Slavina (v.), Prestranek (v.) (leg. B. Horvat 25-may-1986, 714 , n.spec. $=1$ ). 


\section{HYLIDAE}

\section{Hyla arborea}

s.I. (leg. anonim., 30-sep-1959, 355, n.spec. = 1); s.d., 260); (293); 305); (330); 355); (712); 990); (1005); 1308); (1340); 1352); (1355); 1358); (1381); 1433); (1435); 1516); (1622); 1850); (leg. G. Džukić s.d., 355, n.spec. = 25); (1272); (leg. M. Radovanović 08-mar-1959, 1856, n.spec. =1); (leg. $P$. Durbešić 18-jul-1980, 2221, n.spec. =1); Bosnia and Herzegovina Gacko: Jovići (v.) (leg. G. Džukić, 13-jun-1975, 1403, n.spec. = 1); Kupres (leg. G. Džukić, M. Kalezić 17-may-1983, 2748, n.spec. = 1); Mt. Bjelašnica (leg. G. Džukić 04-aug-1970, 710, n.spec. =1); Sarajevo: Kijevo (v.), Železnica river (leg. G. Džukić 12-may-1983, 694/1, n.spec. =2); Croatia Baranja: Batina (v.) (leg. anonim., 08-apr-1972, 532, n.spec. = 5); Koprivnica: Peteranec (v.) (leg. anonim. 05-apr-1988, 715, n.spec. = 5); Osijek: Kopačevski Rit (leg. G. Džukić 28-apr-1970, 710, n.spec. =1); Greece Lamia: Kamena Vourla (leg. L. Đurđević, 10-jul-1979, 354, n.spec. =1); Macedonia Berovo: Berovsko lake, Ratevska river (leg. G. Džukić, 26-may-1970, 710, n.spec. =1); Dojran: Dojransko lake, Nikolić (v.) (leg. G. Džukić, S. Petkovski 22-jun-1995, 215, n.spec. =1); Suva Reka accumulation (leg. anonim. 08-may-1997, 1418, n.spec. =1); Gevgelija (leg. G. Džukić 08-nov-1973, 532, n.spec. $=$ 1); Vardar river bank (leg. G. Džukić 21-jun-1995, 2239, n.spec. =1); Kumanovo - Kriva Palanka: Stracin (v.), lake (leg. G. Džukić, S. Petkovski 24-jun-1995, 215, n.spec. = 1); Mt. Osogovske Planine: Ponikva (leg. G. Džukić, S. Petkovski 23-jun-1995, 215, n.spec. =1); Prespa lake: Ezerani (v.) (leg. anonim. 07-may-1997, 995, n.spec. =2); Struga: Belčišta (v.) (leg. anonim. 06-may-1997, 1422, n.spec. =4); Strumica: Novo Selo (v.), Monospitovo (v.) (leg. G. Džukić 24jun-1998, 266, n.spec. = 4); Strumičko Pole (leg. M. Kalezić, B. Kryštufek 20-apr-1990, 2528, n.spec. = 1); Montenegro Berane [“Ivangrad"]: Petnjica (v.) (leg. Ć. Muratović, 16-apr-1981, 354, n.spec. =6); Grahovo - Vilusi: Velika Osječenica (v.) (leg. anonim. 30-apr-1988, 987, n.spec. =1); Morača: Uljara (leg. Z. Martinović 20-sep-1976, 354, n.spec. = 2); Mt. Durmitor (leg. anonim. 2002, 1829, n.spec. =2); s.d., 682); (1314); Mt. Lukavica: Kapetanovo lake (leg. anonim. 23-aug-2002, 2473, n.spec. = 4); (leg. G. Džukić, M. Kalezić 23-aug-2002, 2491, n.spec. = 1); Mt. Sinjavina: Zminičko lake (leg. anonim. 10-jul-1971, 1630, n.spec. =1); Mt. Žijovo: Rikavačko lake (leg. G. Džukić, M. Kalezić, A. Ivanović 20-sep-1991, 1839, n.spec. = 3); Nikšić (leg. anonim. 14-aug-1976, 354, n.spec. = 1); Rijeka Crnojevića: Podhum (v.) (leg. G. Džukić 11-mar-1974, 1332, n.spec. = 2); Skadarsko lake: Plavica (leg. V. Bušković 07-aug-1984, 354, n.spec. =4); Tivat: Grbaljsko Polje, Tivatske Solane (leg. G. Džukić, I. Aleksić, M. Kalezić, D. Bejaković 07-apr-1992, 1880, n.spec. = 3); Ulcinj (leg. anonim. 01-jun-1975, 354, n.spec. = 2); (leg. H. Babačić 15-aug-1978, 354, n.spec. = 16); Štoj (v.) (leg. G. Pasuljević 23-jun-1976, 354, n.spec. = 5); Vilusi: Banjani, Voluje Oko (leg. G. Džukić, M. Kalezić 05-jun-1984, 2603, n.spec. =1); Virpazar: Orahovačko Polje (leg. G. Džukić, I. Aleksić, D. Bejaković, M. Kalezić 07-apr-1992, 267, n.spec. = 19); (leg. G. Džukić, M. Kalezić, I. Aleksić, D. Bejaković 07-apr-1992, 1519, n.spec. =2); (1631); Orahovštica stream (leg. G. Džukić 04-apr-1989, 2666, n.spec. = 3); Serbia (leg. G. Džukić, M. Kalezić, 14-jun-2006, 2313, n.spec. = 2); Banatska Palanka: Karaš river, Đavolov Most (leg. anonim. 22-mar-1970, 355, n.spec. =1); Bela Crkva (leg. M. Marković s.d., 2326, n.spec. = 41); Dragića Hat, Đavolji Most (leg. G. Džukić, M. Kalezić 19mar-2001, 2324, n.spec. =1); Dupljaja (v.) (leg. M. Kalezić, G. Džukić, M. Marković 19-mar-2001, 1234, n.spec. = 1); Mrtva Nera (leg. G. Džukić, M. Kalezić 05-apr-2003, 1082, n.spec. =2); Blace (leg. N. Miletić 22-jun-1975, 354, n.spec. = 1); Deliblato sands (leg. anonim. 23-may-1999, 2533, n.spec. = 3); Gaj (v.) (leg. G. Džukić, M. Kalezić 18-mar-2005, 2021, n.spec. =1); Majur Bara (leg. anonim. 20-jul1970, 355, n.spec. =1); (leg. G. Džukić 06-apr-1981, 355, n.spec. = 1); 16-may-1976, 355); Utrine (leg. G. Džukić, M. Kalezić 01-aug-1999, 1388, n.spec. = 1); Zubanov Salaš (leg. anonim. 17-jun-1999, 1095, n.spec. = 19); Gnjilane (leg. D. Jović 11-jul-1982, 354, n.spec. =1); (1895); Kosovska Kamenica (leg. B. Milošević 19-jul-1979, 1895, n.spec. = 1); Sveti Jovan (leg. B. Milošević 25-aug-1977, 354, n.spec. = 3); Goleš (leg. D. Milenković 16-jun-1977, 354, n.spec. = 1); Gornji Milanovac: Beršići (v.) (leg. D. Kataranovski, V. Cvetković 17-may-1994, 551, n.spec. =1); Horgoš: Bački Vinogradi (v.), Selevenjske Pustare (leg. M. Kalezić, G. Džukić 11-apr-1998, 226, n.spec. =1); Kladovo: Milutinovac (v.), Balta Lakuri (leg. G. Džukić, M. Kalezić 18-apr-2006, 1203, n.spec. =1); Kosovo (leg. G. Pasuljević s.d., 354, n.spec. =2); Kosovska Mitrovica: Banjska (v.), Banjska river (leg. D. Todorović 28-apr-1978, 354, n.spec. = 1); Lovac (v.) (leg. D. Todorović 02-may-1978, 354, n.spec. = 2); 
Suvi Do (v.) (leg. D. Todorović 28-may-1978, 354, n.spec. =1); Leskovac: Belanovac (v.), Pašina Česma (leg. G. Džukić, M. Kalezić 01-sep-2000, 1246, n.spec. = 2); Gornje Stopanje (v.) (leg. G. Džukić, M. Kalezić 01-sep-2000, 1246, n.spec. =1); Milanovo (v.) (leg. G. Džukić, M. Kalezić 01-sep2000, 1246, n.spec. = 1); Mt. Beljanica (leg. anonim. 17-jul-2004, 1831, n.spec. =1); Mt. Kopaonik: Brezova Glava (leg. J. Crnobrnja-Isailović 01-may-1986, 355, n.spec. = 2); Mt. Rtanj: Rtanj (v.) (leg. G. Džukić 03-may-1977, 355, n.spec. = 1); Mt. Vidlič: Slavinja (v.) (leg. M. Kalezić, G. Džukić 15may-2004, 239, n.spec. =1); Mt. Vršačke Planine: Mesić (v.), Đeram (leg. G. Džukić 30-may-2000, 241, n.spec. = 3); Niš: Mezgraja (v.) (leg. Z. Nikolić 08-oct-1977, 354, n.spec. =1); Obedska Bara: Obrež (v.) (leg. anonim. 30-apr-1992, 1163, n.spec. =1); Ravenica (leg. G. Džukić 11-may-2004, 2321, n.spec. = 1); Pančevo: Opovo (v.), Gergina Slatina (leg. G. Džukić, M. Kalezić 15-apr-2005, 1076, n.spec. = 2); Peć: Đurakovac (v.) (leg. D. Lakušić 23-may-1978, 354, n.spec. = 1); Osojane (v.) (leg. D. Lakušić 10-apr-1978, 354, n.spec. = 2); (leg. H. Babačić 05-oct-1978, 354, n.spec. = 1); Vitomirica (v.) (leg. H. Babačić 10-jul-1978, 354, n.spec. =4); Zobijak (leg. H. Babačić 08-sep-1978, 354, n.spec. = 2); Pešter Plateau: Delimeđe (v.), Stanovi, Karaiska Bara (leg. G. Džukić, M. Kalezić 28-apr-2010, 2683, n.spec. =1); Karajukića Bunari (v.) (leg. J. Crnobrnja-lsailović 28-aug1997, 2669, n.spec. = 1); Tuzinje (v.) (leg. G. Džukić 19-sep-1969, 355, n.spec. = 2); Pirot: Krupac (v.), Krupačko Blato lake (leg. G. Džukić, M. Kalezić 14-may-2004, 1090, n.spec. =1); Pirot - Krupac (v.) (leg. G. Džukić 22-may-1997, 355, n.spec. = 1); Plavna (leg. G. Džukić 09-jun-1971, 355, n.spec. = 1); Požarevac: Salakovac (v.) (leg. anonim. 27-apr-1981, 1866, n.spec. = 1); Priština: Kosovo Polje (leg. R. Rajković 07-sep-1977, 354, n.spec. = 1); Vragolija (v.) (leg. S. Svilenkov 10-may-1982, 1895, n.spec. =1); Priština - Uroševac: Lipljan (leg. N. Živić 28-jun-1981, 354, n.spec. =1); Raška (leg. S. Novićević 01-jun-1977, 354, n.spec. = 30); Smederevo: Provalija (leg. A. Urošević 26-mar2011, 2218, n.spec. = 1); Stari Vlah: Sjenica (leg. G. Džukić, M. Kalezić 27-apr-2010, 2200, n.spec. = 2); Uvac river, Sjenička cave (leg. anonim. 10-aug-1982, 1621, n.spec. = 2); Subotica: Makova Sedmica (v.), Hrastovača (leg. G. Džukić, M. Kalezić 08-jul-1999, 1169, n.spec. = 1); (leg. M. Kalezić, G. Džukić 31-jul-1999, 245, n.spec. = 2); Hrastovača, Čavolj (leg. G. Džukić, M. Kalezić 08-jul-1999, 1109, n.spec. = 14); Hrastovača, Pačarnik and Gater (leg. M. Kalezić, G. Džukić 11-apr-1998, 252, n.spec. = 14); Uroševac (leg. S. Novićević, B. Lalić 14-apr-1976, 354, n.spec. = 2); Užice: Mačkat (v.) (leg. anonim. 02-jun-1991, 2756, n.spec. = 1); Valjevo: Koceljeva, Ljutica (v.), Rasnica (leg. G. Džukić 25-apr-1998, 2381, n.spec. = 1); (leg. G. Džukić, S. Petkovski 25-apr-1998, 213, n.spec. = 1); Zaječar: Grljan (v.), Prličko Blato (leg. G. Džukić, M. Kalezić 25-apr-2009, 1078, n.spec. = 1); Zrenjanin: Ečka (v.) (leg. anonim. 19-oct-1970, 355, n.spec. =1).

\section{Hyla savignyi}

Iraq Baghdad (leg. anonim., 1983, 1838, n.spec. =1).

\section{PELOBATIDAE}

\section{Pelobates fuscus}

S.I. (leg. anonim., s.d., 293, n.spec. = 3); (305); 338); (1308); 1436); (1459); 1613); (2183); 2284); (leg. G. Džukić s.d., 1401, n.spec. = 1); (leg. J. Crnobrnja-Isailović s.d., 618, n.spec. = 16); Croatia Baranja: Bilje (v.) (leg. J. Mikuška, 24-aug-1970, 669 - 2, n.spec. = 1); Kazuksiga (leg. J. Mikuška 02-sep-1970, 669 - 2, n.spec. = 1); Čazma (leg. G. Džukić 01-apr-1976, 1399, n.spec. = 1); Donji Miholjac (leg. D. Kovačić 01-may-1986, 2486, n.spec. = 5); Istra: Motovun, Motovunska Šuma forest - Istarske Toplice spa (leg. M. Kalezić, E. Kletečki 31-mar-1991, 2488, n.spec. = 1); Koprivnica: Peteranec (v.) (leg. anonim. 05-apr-1988, 715, n.spec. =8); Velika Gorica: Turopolje, Orle (v.) (leg. G. Džukić 23-apr-1981, 1434, n.spec. =1); Serbia Aleksinac: Ćićina (v.) (leg. G. Džukić, M. Kalezić, 15-may-2000, 1165, n.spec. = 1); Apatin: Lakatoš (leg. G. Džukić 10-apr-1972, 1179, n.spec. = 2); Bela Crkva: Bela Crkva - Kajtasovo (v.) (leg. G. Džukić, M. Kalezić 19-mar-2001, 1185, n.spec. = 1); Jasenovo (v.) (leg. M. Kalezić, G. Džukić, M. Marković 24-apr-2001, 1374, n.spec. = 2); vicinity (leg. M. Marković s.d., 1216, n.spec. = 10); Beograd: Borča (v.), Mali Zbeg (leg. L. Đurđević 27-apr-1998, 1188, n.spec. = 1); 08-sep-1999, 1183); 24-aug-1999, 1173); Krnjača (v.) (leg. 
M. Kalezić, G. Džukić 14-apr-2006, 218, n.spec. = 2); (leg. S.D. Matvejev 27-mar-1975, 618, n.spec. = 2); Deliblato sands (leg. G. Džukić, M. Kalezić 20-oct-1998, 1168, n.spec. =4); (leg. M. Kalezić, G. Džukić 22-may-1999, 204, n.spec. = 2); Banatska Palanka (leg. V. Malešević 30-aug-2008, 211, n.spec. =1); Deliblato (v.) - Mramorak (v.), Crna Bara (leg. G. Džukić, M. Kalezić 18-mar-2005, 1187, n.spec. = 3); Dubovac (v.), Dubovac (v.), Dubovački Rit (leg. G. Džukić 20-apr-2004, 1172, n.spec. =1); Đurica, Stevanove Ravnice and mali Pesak (leg. G. Džukić, M. Kalezić 21-aug-1998, 1178, n.spec. = 2); Tuzla (leg. M. Kalezić, G. Džukić 20-may-1998, 261, n.spec. =6); Gaj (v.) (leg. G. Džukić, M. Kalezić 18-mar-2005, 2023, n.spec. =2); Majur Bara (leg. G. Džukić 20-jul-1970, 1393, n.spec. = 5); 16-may1976, 618); Mramorak (v.), Kraljevac, Obzovik Bara, Vodice churches (leg. G. Džukić, M. Kalezić 25-mar-2005, 1167, n.spec. =1); Šumarak (v.) - Grebenac (v.) (leg. G. Džukić, M. Kalezić 19-mar2001, 2142, n.spec. = 2); Utrine (leg. G. Džukić, M. Kalezić 16-sep-1999, 1189, n.spec. =9); 01-aug1999, 1175); Knjažev Salaš (leg. M. Kalezić 17-apr-2004, 209, n.spec. =1); Salaš Vlaški (leg. M. Kalezić, G. Džukić 26-jun-1999, 232, n.spec. = 6); Jagodina: Staro Lanište (v.) (leg. G. Džukić, M. Kalezić 02-sep-2000, 1170, n.spec. =1); Kladovo: Lolića Rit (leg. G. Džukić, M. Kalezić 17-may-2000, 1182 , n.spec. =2); Kovin (leg. G. Džukić 25-aug-1975, 618, n.spec. =1); Crna Bara (leg. G. Dżukić, $M$. Kalezić 25-mar-2005, 1167, n.spec. =1); (leg. M. Kalezić, G. Džukić 25-mar-2005, 209, n.spec. =1); Crvenka, Rimski Šanac (leg. G. Džukić, M. Kalezić 18-mar-2005, 1166, n.spec. =2); Gaj (v.) (leg. G. Džukić, M. Kalezić 18-mar-2005, 1027, n.spec. = 1); Novi Bečej: Slano Kopovo ["Lesino Kopovo"] (leg. G. Džukić 19-may-1976, 618, n.spec. = 14); (leg. G. Džukić, M. Kalezić 18-apr-1990, 1190, n.spec. = 20); Obedska Bara: Obrež (v.), Ravenica (leg. G. Džukić, M. Kalezić 30-apr-1992, 1370, n.spec. = 4); Pančevo: Ivanovo (v.), Danube Embankment (leg. G. Džukić, M. Kalezić 02-apr-2002, 1174, n.spec. =1); Sremski Karlovci: Čortanovci (v.), Čortanovačka Plaža beach (leg. M. Milenković 27-mar-1992, 1840, n.spec. = 1); Karlovački Vinogradi station (leg. M. Kalezić, G. Džukić 31-oct-2004, 218, n.spec. = 1); Srpska Crnja: Radojevo (v.) (leg. A. Rafeski 10-jul-1995, 2391, n.spec. $=2$ ); Subotica (leg. anonim. 21-mar-1976, 343, n.spec. = 1); 13-mar-1977, 338); (2183); Hajdukovo (v.) (leg. J. Mikuška 20-aug-1966, 618, n.spec. =1); Makova Sedmica (v.), Hrastovača (leg. G. Džukić, M. Kalezić 08-jul-1999, 1169, n.spec. = 8); 23-mar-2000, 1181/1); 1181/2); (leg. M. Kalezić, G. Džukić 13-mar-1997, 254, n.spec. = 10); 09-apr-1998, 229); 31-jul-1999, 233); 23-mar-2000, 207); 18-aug-1997, 264); Hrastovača - Varna (leg. M. Kalezić, G. Džukić 15-mar-1997, 1862, n.spec. = 1); Hrastovača, Cirkuzantska Bara (leg. G. Džukić 23-mar-1996, 1177, n.spec. = 6); Hrastovača, Čavolj (leg. M. Kalezić, G. Džukić 31-jul-1999, 212, n.spec. = 7); 02-oct-1998, 258); Hrastovača, Pačarnik (leg. G. Džukić, M. Kalezić 10-apr-1998, 1176, n.spec. = 1); Hrastovača, Pačarnik and Gater (leg. M. Kalezić, G. Džukić 11-apr-1998, 252, n.spec. =1); Zrenjanin: Samoš (v.) - Dobrica (v.) (leg. G. Džukić, M. Kalezić 03-jun-2000, 1180, n.spec. =3); Slovenia Murska Sobota: Radenci (v.), Turjanci (v.) (leg. anonim., 01-apr-1988, 1398, n.spec. $=1$ ).

\section{Pelobates syriacus}

Macedonia Gevgelija (leg. G. Džukić, 08-nov-1973, 532, n.spec. =1); Kočani: Pribačevo (v.) (leg. G. Džukić 09-may-1997, 2292, n.spec. =1); Prespa lake: Ezerani (v.) (leg. anonim. 07may-1997, 1420, n.spec. = 1); Skoplje: Petrovec (v.) (leg. M. Kalezić 23-sep-1981, 202, n.spec. =6); Strumica: Bosilovo (v.) - Sekirnik (v.) (leg. G. Džukić 19-may-1980, 648, n.spec. =12); Deliblato sands (leg. M. Kalezić, G. Džukić, 26-jun-1999, 234, n.spec. = 2); 20-oct-1998, 253); Devojačko Brdo (leg. M. Kalezić, G. Džukić 22-may-1999, 216, n.spec. = 5); Đurica, Stevanove Ravnice (leg. anonim. 22-may-1999, 243, n.spec. = 5); (leg. G. Džukić 08-jul-1998, 1184, n.spec. = 4); (leg. M. Kalezić, G. Džukić 22-jun-1998, 230, n.spec. = 3); Tuzla (leg. M. Kalezić, G. Džukić 20-may-1998, 231, n.spec. = 23); Gaj (v.) (leg. G. Džukić, M. Kalezić, A. Ivanović, M. Slijepčević, A. Urošević 10-jun-2012, 1273 , n.spec. = 31); Kajtasovo (v.), Kajtaski Vinogradi (leg. anonim. 29-jun-1988, 251, n.spec. =2); Majur Bara (leg. anonim. 29-apr-1980, 251, n.spec. =1); 05-apr-1981, 251); (leg. G. Džukić 16-may1976, 251, n.spec. =1); 25-aug-1975, 251); 28-aug-1987, 251); Utrine (leg. G. Džukić, M. Kalezić 01aug-1999, 1171, n.spec. = 7); (leg. M. Kalezić, G. Džukić 16-jun-1999, 243, n.spec. =10); 17-jun-1999, 240); Knjažev Salaš (leg. G. Džukić, M. Kalezić 17-apr-2004, 1214, n.spec. =1); (leg. M. Kalezić 17-apr2004, 209, n.spec. =1); Jagodina: Staro Lanište (v.) (leg. G. Džukić, M. Kalezić 02-sep-2000, 1170, n.spec. = 3); Kikinda: vicinity (leg. anonim. 01-aug-1987, 251, n.spec. =1); Kladovo: Lolića Rit (leg. G. Džukić, M. Kalezić 17-may-2000, 1182, n.spec. =1); Pančevo: Ivanovo (v.) (leg. G. Džukić 02apr-1980, 251, n.spec. = 13); 29-aug-1987, 251). 


\section{RANIDAE}

\section{Pelophylax esculentus complex}

s.l. (leg. anonim., 20-oct-1994, 631, n.spec. = 39); s.d., 305); (320); 331); (336); 337); (338); 340); (342); 347); (349); 560); (567); 571); (574); 579); (585); 586); (587); 588); (589); 591); (596); 597); (598); 604); (606); 607); (608); 610); (612); 613); (614); 615); (617); 624); (626); 627); (656); 664); (681); 696); (706); 990); (1312); 1341); (1346); 1361); (1435); 2183); 609/1); 609/2); (leg. G. Džukić s.d., 346, n.spec. = 17); (613); 620); (636); 712); (1303); 1308); (leg. V. Gavrilović s.d., 672, n.spec. =9); Bosnia and Herzegovina Bosansko Grahovo: Korana stream, Pišteljak (leg. anonim., 09apr-1989, 2662, n.spec. =1); Gacko: Gatačko Polje (leg. G. Džukić 13-jun-1975, 654, n.spec. =1); Jovići (v.) (leg. G. Džukić 13-jun-1975, 1403, n.spec. = 3); Tuzla: Donje Dubrave (v.) (leg. G. Džukić 06-jun-1985, 594, n.spec. = 10); Croatia Baranja (leg. J. Mikuška, 01-apr-1971, $669-1$, n.spec. = 3); Batina (v.) (leg. anonim. 08-apr-1972, 532, n.spec. = 4); Koha (leg. J. Mikuška 04-may1971, $669-1$, n.spec. = 1); Sakadaš lake (leg. J. Mikuška 25-nov-1970, $669-1$, n.spec. = 2); 07-jan1971, $669-1$ ); Tikveš (v.) (leg. J. Mikuška 26-aug-1970, $669-1$, n.spec. = 2); Cres (ins.): Cres (leg. N. Tvrtković 07-apr-1976, 705, n.spec. $=2$ ); Mt. Velebit (leg. anonim. 1955, 1313, n.spec. $=4$ ); Obrovac (leg. G. Džukić, M. Kalezić 02-jun-1985, 2241, n.spec. = 3); Otočac: Švica (v.) (leg. G. Džukić, M. Kalezić 12-apr-1989, 1394, n.spec. = 1); Ravni Kotari: Ždralovac (leg. N. Tvrtković 11-jun1977, 1463, n.spec. = 2); Varaždin: Donja Voća (v.) (leg. B. Jalžić 18-jul-1976, 1343, n.spec. = 1); Velika Gorica: Turopolje, Orle (v.) (leg. G. Džukić 20-may-1987, 1853, n.spec. =2); Vukomeričke Gorice, Dragonožec (v.), Trpuci (leg. N. Tvrtković 18-apr-1976, 707, n.spec. =4); Zagreb (leg. $D$. Pelić, G. Džukić 28-apr-1975, 693, n.spec. = 5); Dubrava (v.), Vrbovec (leg. G. Džukić 30-jun-1976, 550, n.spec. =1); Maksimir (leg. Z. Krišković 1963, 532, n.spec. =1); Greece Halkidiki: Stavros, Olimpijada (v.) (leg. M. Marković, 15-jul-2003, 1431, n.spec. = 5); Macedonia Katlanovo: Pčinja river (leg. anonim., 23-jun-1998, 301, n.spec. = 2); Kočani: Pribačevo (v.) (leg. anonim. 09-may1997, 687, n.spec. = 5); (leg. G. Džukić 09-may-1997, 584, n.spec. = 25); (593); Mt. Galičica (leg. G. Džukić 03-may-1997, 2682, n.spec. = 2); Prespa lake: Ezerani (v.) (leg. G. Džukić 07-may-1997, 611, n.spec. = 13); (2687); Struga: Belčišta (v.), Sinji Virovi (leg. G. Džukić 06-may-1997, 262, n.spec. = 20); (603); 667); (685); Montenegro Berane [“Ivangrad"] (leg. M. Račić, 15-sep-1979, 1517, n.spec. =1); Lubnice (v.) (leg. R. Lević 1978, 560, n.spec. =2); Danilovgrad: Lazine, Jastreb (leg. G. Džukić, M. Kalezić 26-apr-2002, 2327, n.spec. = 2); Grahovo: Kotlići (v.) (leg. anonim. 15-jun-1983, 1311, n.spec. =2); Herceg Novi: Sutorina (v.) (leg. A. Hegediš 10-aug-1990, 1490, n.spec. =2); Mt. Durmitor: Riblje lake (leg. G. Džukić 21-jul-1975, 1630, n.spec. =2); Mts Prokletije: Mt. Bogićevica, Babino Polje (leg. G. Pasuljević 01-aug-1980, 560, n.spec. =3); Plav (leg. G. Pasuljević 22-aug-1980, 1517, n.spec. =1); Rijeka Crnojevića: Ceklin (v.), Pijavica spring (leg. G. Džukić, M. Kalezić 26-aug-2002, 2302, n.spec. = 1); Podhum (v.) (leg. G. Džukić 10-mar-1974, 1332, n.spec. =1); Skadarsko lake (leg. anonim. 01-jun-1991, 623, n.spec. = 8); 01-oct-1992, 629); (leg. G. Džukić 01oct-1992, 650, n.spec. = 3); (651); Beška (ins.) (leg. anonim. 08-jun-1991, 1833, n.spec. =1); (leg. G. Džukić 04-jun-1990, 2677, n.spec. =1); (leg. G. Džukić, I. Aleksić, D. Bejaković, M. Kalezić 15-may-1994, 1627, n.spec. = 2); Grmožur (ins.) (leg. G. Džukić 25-jul-1975, 699, n.spec. =1); Krajina, Gornja Briska (v.) (leg. G. Džukić, M. Kalezić 21-apr-1991, 1430, n.spec. = 5); Kostanica (v.) (leg. anonim. 25apr-1991, 1478, n.spec. = 2); Murići (v.) (leg. anonim. 15-jun-1997, 645, n.spec. = 5); Krnjice (v.) (leg. anonim. 05-jun-1991, 1833, n.spec. = 2); Vranjina (ins.) (leg. G. Džukić 09-jul-1969, 613, n.spec. = 3); (leg. G. Džukić, I. Aleksić, D. Bejaković, M. Kalezić 17-may-1994, 1504, n.spec. =2); Tivat: Luštica, Žanjic (v.) (leg. G. Džukić 27-apr-2002, 2335, n.spec. =2); Tivatske Solane (leg. V. Vasić 06-jul1974, 680, n.spec. = 2); (1345); Lješevići (v.) (leg. A. Hegediš 08-apr-1990, 987, n.spec. =1); Ulcinj (leg. anonim. 01-jun-1975, 354, n.spec. =1); Donji Štoj (v.) (leg. G. Džukić 02-apr-1989, 2684, n.spec. = 9); Štoj (v.) (leg. G. Pasuljević 23-jun-1976, 2804, n.spec. = 1); Virpazar (leg. anonim. 01-apr-1992, 686, n.spec. = 13); 03-apr-1992, 616); 621-1); 621-2); 621-3); (leg. G. Džukić, M. Kalezić 04-apr-1980, 649, n.spec. = 3); 03-apr-1992, 580); Crmnica (leg. anonim. 14-jun-1997, 642, n.spec. = 5); (leg. G. Džukić 15-jun-1997, 575, n.spec. = 8); Orahovačko Polje (leg. anonim. 07-apr-1992, 1894, n.spec. $=$ 6); (leg. G. Džukić, I. Aleksić, D. Bejaković, M. Kalezić 07-apr-1992, 590, n.spec. = 7); Serbia (leg. anonim., s.d., 339, n.spec. $=11$ ); (341); 343); (581); 582); (601); 1884); 625-1); 625-2); (leg. G. Pasuljević s.d., 2804, n.spec. =24); Aleksandrovac: Vrbnica (v.) (leg. G. Džukić 02-jun-1977, 1884, n.spec. =4); 
Banatska Palanka: Labudovo Okno (leg. V. Kalafatić 11-may-1994, 1483, n.spec. =1); Banatski Karlovac: Seleuš (v.) - Ilandža (v.) (leg. G. Džukić, M. Kalezić 20-mar-2001, 657, n.spec. =1); Bela Crkva: Kusić (v.) (leg. G. Džukić, M. Kalezić 01-nov-2002, 676, n.spec. = 3); Beograd: Borča (v.) (leg. anonim. 02-may-1994, 1163, n.spec. =1); Dobanovci (v.) (leg. G. Džukić, M. Kalezić 22-may1993, 1445, n.spec. = 8); Blace (leg. G. Pasuljević 20-sep-1976, 2797, n.spec. $=1$ ); Deliblato sands (leg. G. Džukić, M. Kalezić 01-jun-1998, 294, n.spec. =1); Dubovac (v.), Dubovac (v.), Dubovački Rit (leg. anonim. 03-sep-2003, 670, n.spec. $=20$ ); (leg. I. Krizmanić, M. Kalezić, Lj. Tomović 30-sep-2003, 1900, n.spec. $=14$ ); Dulin Bara (leg. anonim. 28-jul-2004, 638, n.spec. $=20$ ); 05-oct-2003, 637); Đurica, Stevanove Ravnice (leg. A. Ivanović, M. Kalezić 29-may-2004, 1101, n.spec. =9); Stevanove Ravnice, Velika Bara (leg. anonim. 03-sep-2003, $668-1$, n.spec. =18); $668-2$ ); Gaj (v.), Hatarice (leg. G. Džukić, M. Kalezić, A. Ivanović, M. Slijepčević, A. Urošević 10-jun-2012, 1273, n.spec. = 2); Konstantinova Bara (leg. G. Džukić, M. Kalezić 27-oct-2001, 1091, n.spec. = 5); Lokva (leg. I. Krizmanić 29-may-2004, 663 - 1, n.spec. =15); 663 - 2); Majur Bara (leg. G. Džukić 16-may-1976, 346, n.spec. = 3); Šumarak (v.) - Grebenac (v.) (leg. G. Džukić, M. Kalezić 18-mar-2001, 655, n.spec. = 1); Utrine (leg. anonim. 26-jun-1999, 561, n.spec. = 4); (leg. G. Džukić, M. Kalezić 04-sep-1999, 660, n.spec. = 3); 28-jul-2004, 1217); Zubanov Salaš (leg. G. Džukić 17-jun-1999, 665, n.spec. $=22$ ); Zamfir Bara (leg. anonim. 26-jun-1999, 561, n.spec. = 1); (leg. G. Džukić, M. Kalezić 25-jun-1999, 1107, n.spec. $=2$ ); Donji Milanovac (leg. anonim. 28-jun-2003, 640, n.spec. $=20$ ); 01-may-2004, 641); Đerdap gorge: Trajanov Most (leg. G. Džukić, M. Kalezić 12-jun-1998, 2682, n.spec. = 1); Glogovac: Drenica (v.) (leg. G. Pasuljević 18-jun-1977, 2797, n.spec. = 2); (leg. I. Petrov 18-jun1977, 1515, n.spec. = 1); Gnjilane: Kosovska Kamenica, Ograđe (leg. B. Miloševic 06-jul-1979, 1517, n.spec. =4); Sveti Jovan (leg. G. Pasuljević 20-aug-1977, 2803, n.spec. =2); Goleš (leg. anonim. 1979, 560, n.spec. = 2); Inđija: Beška (v.) (leg. anonim. s.d., 605, n.spec. = 13); Jagodina: Staro Lanište (v.), Nova Bara (leg. G. Džukić, M. Kalezić 14-may-2000, 600, n.spec. =1); Kladovo (leg. I. Krizmanić 27-jun-2003, 635, n.spec. = 32); Kosovo: Kišnica (v.) (leg. B. Vlajčetić 18-may-1984, 558, n.spec. = 1); Kosovska Mitrovica: Banjska (v.) (leg. D. Todorović 24-mar-1981, 1517, n.spec. = 5); (leg. G. Pasuljević 28-apr-1978, 2798, n.spec. = 4); Krnjane, Ibar river (leg. G. Pasuljević 18-jun-1973, 2797, n.spec. = 6); (2803); Leposavić (leg. G. Pasuljević 06-may-1973, 2798, n.spec. =2); Kamenica (v.) [“Kamešnička Rožka”] (leg. G. Pasuljević 07-aug-1978, 2803, n.spec. = 2); Zubin Potok, Lučka river (leg. G. Pasuljević 02-oct-1976, 2804, n.spec. =1); Zvečan (v.) (leg. G. Pasuljević 15-may-1977, 2803, n.spec. = 3); Kraljevo (leg. I. Krizmanić 03-may-2004, 666, n.spec. =10); Žiča (v.) (leg. Z. Stojanović 29-sep-1982, 558, n.spec. =1); (1895); Kruševac (leg. anonim. 22-may-2003, 688-1, n.spec. = 18); 688-2); (leg. G. Pasuljević 13-sep-1976, 2797, n.spec. =2); Kuršumlija: Prolom Banja spa (leg. G. Pasuljević 11-jun-1979, 1517, n.spec. = 4); Leskovac: Vučje, Manetove Livade (leg. G. Džukić, M. Kalezić 16-jun-2001, 1105, n.spec. = 1); Žabljani (v.) (leg. I. Krizmanić 21-may-2003, 671 - 1, n.spec. = 11); 671 - 2); Ljubovija: Gornja Trešnjica (v.) (leg. J. Crnobrnja-Isailović, I. Aleksić 22-mar1999, 662, n.spec. =1); Trešnjica (v.) (leg. G. Džukić 27-may-1972, 613, n.spec. =1); Majdanpek: Gornjane (v.), Ljubova river (leg. K. Bajić 10-jul-1990, 2690, n.spec. =2); Železnik (v.) - Debeli Lug (v.) (leg. V. Kalafatić 26-may-1980, 1633, n.spec. =1); Medveđa (leg. J. Talić 01-may-1981, 1895, n.spec. = 1); Mt. Fruška Gora: Testera (leg. G. Džukić 06-jun-1976, 1884, n.spec. = 3); Mt. Kopaonik: Jošanička Banja spa (leg. G. Džukić 27-oct-1973, 613, n.spec. = 3); (leg. O. Glišić 30apr-1986, 613, n.spec. = 3); Mt. Miroč: Cvetanovac (leg. G. Džukić, M. Kalezić 17-may-2000, 1387, n.spec. = 1); Mt. Skopska Crna Gora: Letnica (v.) (leg. G. Pasuljević 08-aug-1979, 1517, n.spec. = 8); Mt. Vršačke Planine: Mesić (v.) (leg. anonim. 18-may-1996, 646, n.spec. = 2); Mts ŠarPlanina (leg. G. Pasuljević 1970, 560, n.spec. =15); Negotin (leg. I. Krizmanić 27-jun-2003, $673-2$, n.spec. = 12); 02-may-2004, $673-1$ ); (leg. M. Kalezić, G. Džukić 16-may-2004, 690, n.spec. =1); Niš (leg. I. Krizmanić 21-may-2003, $674-1$, n.spec. = 12); 674 - 2); Novi Banovci - Slankamen: Belegiš (v.) (leg. I. Petrov 21-jul-1974, 588, n.spec. = 1); Novi Sad (leg. anonim. 21-mar-1977, 343, n.spec. = 7); Krčedin (v.), Krčedinska Ada (ins.) (leg. G. Džukić 30-apr-1980, 1089, n.spec. = 2); Obedska Bara (leg. I. Krizmanić 18-may-2004, 678, n.spec. = 19); Obrež (v.) (leg. G. Džukić, M. Kalezić 11-may-2004, 2007, n.spec. = 6); Ravenica (leg. anonim. 30-apr-1992, 619, n.spec. = 8); (leg. G. Džukić, M. Kalezić 30-apr-1992, 602, n.spec. = 5); Orahovac: Miruša river (leg. G. Pasuljević, B. Milošević 01-jul-1980, 558, n.spec. =1); Pančevo: Ivanovo (v.) (leg. D. Miljković 11-aug-2006, 1075, n.spec. =1); Pančevački Rit, Crvenka canal (leg. P. Cakić 23-mar-1990, 1031, n.spec. = 2); Pčinja (leg. G. Dżukić 05-jun-1980, 617, n.spec. = 1); Peć (leg. G. Pasuljević 29-sep-1976, 2803, n.spec. = 3); 
Dečani, Visoki Dečani monastery (leg. G. Pasuljević, G. Džukić, B. Milošević 17-jul-1980, 1517, n.spec. =1); Đurakovac (v.), Osojane (v.) (leg. G. Pasuljević 10-oct-1978, 2804, n.spec. = 1); Istok (v.) (leg. G. Pasuljević 25-nov-1970, 2804, n.spec. =1); Vitomirica (v.) (leg. B. Popović 29-oct-1982, 558, n.spec. = 2); (leg. O. Papović 29-oct-1982, 1895, n.spec. = 3); Pešter Plateau: Karajukića Bunari (v.) (leg. J. Crnobrnja-Isailović 28-aug-1997, 2028, n.spec. =1); (2669); Pirot: Krupac (v.), Krupačko Blato lake (leg. G. Džukić 22-may-1977, 1884, n.spec. = 1); Podujevo (leg. G. Pasuljević 20-may-1978, 2797, n.spec. = 2); Prijepolje: Jabuka (v.) (leg. G. Džukić 12-mar-1980, 346, n.spec. =1); Priština (leg. D. Rondić 15-sep-1982, 1897, n.spec. = 1); (leg. G. Pasuljević 13-may-1983, 557, n.spec. =9); (1897); Belaćevac (leg. G. Pasuljević 17-aug-1975, 2798, n.spec. =7); Janjevo (v.) (leg. B. Talović 08-may-1984, 1895, n.spec. = 1); (leg. G. Pasuljević 15-nov-1977, 2796, n.spec. = 28); (leg. V. Talović 08may-1984, 558, n.spec. = 1); Kosovo Polje (leg. D. Čukić 16-aug-1982, 1895, n.spec. = 1); (leg. M. Dimić 15-jun-1982, 1897, n.spec. = 1); (leg. M. Dinić 15-jun-1982, 558, n.spec. =1); Mramor (v.) (leg. G. Pasuljević 03-jul-1979, 583, n.spec. =10); Mt. Grmija (leg. G. Pasuljević 20-oct-1976, 2803, n.spec. = 1); (leg. O. Papović 14-jun-1982, 1895, n.spec. =1); Rzav river (leg. anonim. 2002, 563, n.spec. = 12); Slankamen (leg. G. Džukić 10-jul-1974, 588, n.spec. = 2); Smederevo: Šalinac (v.) (leg. anonim. 10-sep-2003, 639-1, n.spec. =15); 639-2); Sremska Mitrovica: Kuzmin (v.) (leg. G. Pasuljević 03aug-1978, 2797, n.spec. = 2); Srpska Crnja: Radojevo (v.) (leg. A. Rafeski 01-jul-1994, 1890, n.spec. = 1); Stari Vlah: Sjenica, Čedovo (v.) (leg. G. Pasuljević 23-jul-1976, 2798, n.spec. = 7); Subotica (leg. anonim. 06-mar-1977, 338, n.spec. =4); 21-mar-1977, 343); Hajdukovo (v.) (leg. J. Mikuška 09-aug-1965, 1884, n.spec. = 1); Makova Sedmica (v.), Hrastovača, Cirkuzantska Bara (leg. M. Kalezić, G. Džukić 07-mar-1998, 257, n.spec. = 1); Hrastovača, Cirkuzantska Bara and Čavolj (leg. G. Džukić, M. Kalezić 08-jul-1999, 568, n.spec. =29); Hrastovača, Čavolj (leg. G. Džukić, M. Kalezić 08-jul-1999, 1109, n.spec. = 1); Palić (v.) (leg. anonim. 18-apr-1977, 343, n.spec. = 2); Mali Pijac (v.) (leg. J. Mikuška 01-may-1963, 358, n.spec. = 3); vicinity (leg. B. Walwajter 01-sep-1992, 1522, n.spec. = 1); Svilajnac (leg. anonim. 19-sep-2003, 643-1, n.spec. = 13); 643-2); Šabac: Vlasenica (v.), Mićina and Đokina Bara (leg. G. Džukić 14-may-1993, 2095, n.spec. = 2); Trgovište (leg. G. Džukić, M. Kalezić 12-may-1997, 634, n.spec. = 15); Trstenik: Donja Crnišava (v.) (leg. anonim. 19jul-1998, 646, n.spec. = 3); (leg. K. Ljubisavljević 28-jul-1997, 684, n.spec. = 7); 05-aug-1997, 298); Uroševac (leg. G. Pasuljević 12-nov-1976, 2790, n.spec. =17); (2804); (leg. S. Novićević 12-nov-1976, 1869, n.spec. = 1); Dulje (v.) - Crnoljevo (v.) (leg. G. Pasuljević 06-sep-1979, 1517, n.spec. = 3); Štimlje (v.) (leg. G. Pasuljević 20-sep-1976, 2803, n.spec. = 7); Zaječar: Vratarnica (v.) (leg. anonim. 26-sep-2003, 644 - 2, n.spec. = 6); Zrenjanin: Ečka (v.) (leg. anonim. 19-oct-1970, 613, n.spec. = 1); Sečanj (v.), Neuzina (v.) (leg. anonim. 16-may-2003, 2472, n.spec. =2); Tomaševac (v.) (leg. G. Džukić, M. Kalezić 16-may-2003, 658, n.spec. = 1); Zubin Potok (leg. D. Todorović 06-jun1978, 1517, n.spec. =1); Slovenia Brežice: Brežina (v.), Cizelj (leg. B. Horvat, 11-jun-1986, 726, n.spec. = 1); Kostanjevica Na Krki (v.), Krakovski Gozd (leg. J. Gregori 28-mar-1974, 1338, n.spec. = 1); Črnomelj: Vinica (v.) (leg. B. Ktyštufek 07-jun-1972, 959, n.spec. = 1); Kranj: Cerklje (v.) (leg. B. Kryštufek 18-jun-1972, 2678, n.spec. = 3); 20-jun-1972, 959); Tenetiše (v.) (leg. G. Džukić, B. Kryštufek 01-may-1972, 1487, n.spec. = 1); Ljutomer: Žerovinci (v.) (leg. G. Džukić 31-mar-1988, 723, n.spec. = 1); Murska Sobota: Radenci (v.), Turjanci (v.) (leg. anonim. 01-apr-1988, 1398, n.spec. = 2); Novo Mesto: Mirna (v.), Lavrica (leg. J. Gregori, B. Horvat 10-jun-1986, 714, n.spec. = 2); Šentjernej (v.), Grobeljska Gmajna (leg. B. Horvat 23-apr-1986, 714, n.spec. =2).

\section{Pelophylax kurtmuelleri}

Macedonia Dojran: Dojransko lake (leg. G. Džukić, S. Petkovski, 22-jun-1995, 215, n.spec. = 1); Stari Dojran (leg. G. Džukić 08-may-1997, 987, n.spec. =1); Gevgelija (leg. G. Džukić, S. Petkovski 21-jun-1995, 215, n.spec. $=2$ ).

\section{Pelophylax ridibundus}

S.I. (leg. anonim., s.d., 304, n.spec. =2); (319); 531); (1454); (leg. G. Džukić s.d., 358, n.spec. =1); Dobri Do (leg. R. Šupić 13-may-1980, 2143, n.spec. =1); Bosnia and Herzegovina Gacko (leg. anonim., 03-aug-1970, 1885, n.spec. =1); Croatia Velika Gorica: Turopolje, Orle (v.) (leg. G. 
Džukić, 23-apr-1981, 1850, n.spec. = 1); Macedonia Berovo: Berovsko lake, Ratevska river (leg. anonim., 26-may-1970, 290, n.spec. =1); Gradsko: Stobi, Crna (leg. anonim. 13-apr-1972, 535, n.spec. =1); Strumica: Suvi Laki (leg. anonim. 29-may-1970, 290, n.spec. =1); Valandovo: Okno (leg. G. Džukić 07-nov-1973, 532, n.spec. = 1); Montenegro Grahovo - Vilusi: Velika Osječenica (v.) (leg. G. Džukić, 25-aug-1982, 2495, n.spec. =2); 29-aug-2002, 2332); 31-may-1990, 1851); Mt. Bioč: Malo Stabansko lake (leg. G. Džukić, M. Kalezić 06-jun-2008, 2233, n.spec. =2); Skadarsko lake: Krajina, Livari (v.) (leg. J. Crnobrnja-Isailović, I. Aleksić 29-may-1996, 200, n.spec. = 10); Virpazar: Orahovačko Polje (leg. anonim. 07-apr-1992, 572, n.spec. $=5$ ); Serbia (leg. anonim., s.d., 2143, n.spec. = 4); Banatski Brestovac: Ponjavica (leg. G. Džukić, M. Kalezić 23-may-2004, 2008, n.spec. =1); Beograd: Borča (v.), Mali Zbeg (leg. L. Đurđević 24-oct-1999, 1411, n.spec. =1); Veliko Selo (v.), Ada Štefanac (ins.) (leg. P. Cakić 14-nov-1995, 2055, n.spec. =1); Brodarevo: Vrbnica (v.) (leg. G. Džukić 07-jul-1969, 358, n.spec. =1); Ćuprija: Ravanica river (leg. anonim. 30-apr-1992, 348, n.spec. = 3); Deliblato sands: Deliblato (v.) - Mramorak (v.) (leg. G. Džukić, M. Kalezić 18-mar-2005, 1083, n.spec. = 1); Đakovica (leg. S. Šalja 23-jul-1980, 2143, n.spec. =7); Babaj Boks (v.) (leg. G. Pasuljević, G. Džukić, B. Milošević 18-jul-1980, 2143, n.spec. = 3); Gnjilane: Kosovska Kamenica (leg. B. Milošević 04-aug-1979, 2143, n.spec. = 3); Berivojce (v.) (leg. B. Milošević 29-apr-1979, 2143, n.spec. = 1); Ograđe (leg. B. Milošević 27-aug-1978, 2143, n.spec. = 3); Klina: Volujak (v.) (leg. G. Pasuljević, B. Milošević 01-jul-1980, 2143, n.spec. =1); Kosovska Mitrovica: Banjska (v.) (leg. D. Todorović 13-jun-1981, 2143, n.spec. = 5); Rudine (v.) (leg. D. Todorović 06-jun-1979, 2143, n.spec. =1); Kovin (leg. G. Džukić 1969, 358, n.spec. =4); 01-jun-1969, 358); Leskovac: Belanovac (v.), Pašina Česma (leg. G. Džukić, M. Kalezić 01-sep-2000, 1246, n.spec. =1); Mt. Beljanica (leg. anonim. 17-jul-2004, 1831, n.spec. =2); Rečke (leg. G. Džukić 14-jul1998, 1828, n.spec. = 4); Mt. Jastrebac: Buci (v.) (leg. G. Džukić 17-oct-1977, 358, n.spec. =2); Mt. Tara: Šljivovica (v.) (leg. anonim. 15-may-1972, 358, n.spec. =1); Mt. Zlatar: Akmačići (v.) (leg. J. Crnobrnja-Isailović 19-aug-1996, 1092, n.spec. = 1); Mts Šar-Planina: Dragaš, Rapča (v.) (leg. M. Hajredini 22-mar-1980, 2143, n.spec. =1); Nova Varoš: Kokin Brod (v.), Neškoviće (v.), Šupljica (leg. G. Džukić 11-aug-1982, 358, n.spec. = 1); Pančevo: Ivanovo (v.) (leg. B. Mićković 27-jul-1994, 1602, n.spec. =1); Peć (leg. V. Šabović 20-may-1979, 2143, n.spec. =2); Vitomirica (v.) (leg. V. Šabović 12-jun-1979, 2143, n.spec. = 1); Pirot: Krupac (v.), Krupačko Blato lake (leg. G. Džukić, $M$. Kalezić 14-may-2004, 1090, n.spec. =2); Priština: Mramor (v.) (leg. G. Pasuljević 03-jul-1979, 2143, n.spec. = 7); Mt. Grmija (leg. V. Šabović 10-jun-1979, 2143, n.spec. =1); Stari Vlah: Sjenica (leg. G. Džukić, M. Kalezić 27-apr-2010, 2200, n.spec. =1); Subotica: Hajdukovo (v.) (leg. J. Mikuška 09aug-1965, 358, n.spec. = 1); Ludaško lake (leg. J. Mikuška 28-apr-1958, 358, n.spec. =1); Makova Sedmica (v.), Hrastovača, Čavolj (leg. G. Džukić, M. Kalezić 31-jul-1999, 1098, n.spec. = 2); (leg. M. Kalezić, G. Džukić 31-jul-1999, 212, n.spec. = 1); Uroševac (leg. R. Savić 28-may-1979, 2143, n.spec. = 5); Nerodimlje (v.) (leg. D. Marković 07-sep-1980, 2143, n.spec. =1); Valjevo: Gola Glava (v.) (leg. G. Džukić, M. Kalezić 24-aug-1997, 296, n.spec. =2); Petnica (v.), Banja river (leg. M. Nikčević 09-feb-1992, 1875, n.spec. = 1); Slovenia Ljubljana: Ig (v.), vicinity (leg. G. Džukić, 01-apr-1979, 1820 , n.spec. $=1)$.

\section{Pelophylax schquipericus}

Montenegro Virpazar (leg. G. Džukić, M. Marković, M. Kalezić, 22-jun-2004, 2001, n.spec. =11); (2002); 2005); Orahovačko Polje (leg. G. Džukić, I. Aleksić, M. Kalezić, D. Bejaković 07-apr-1992, 1893, n.spec. $=5$ ).

\section{Rana arvalis}

S.I. (leg. anonim., s.d., 531, n.spec. $=4$ ); (2685); Croatia Sisak: Odra river (leg. N. Tvrtković, 23-mar-1977, 1463, n.spec. = 2); Velika Gorica: Peščenica (v.) (leg. N. Tvrtković 24-mar-1977, 1463, n.spec. = 2); Zagreb: Dubrava (v.) (leg. G. Džukić 01-jun-1976, 1399, n.spec. =6); Gornji Stenjevec (leg. N. Tvrtković 11-jan-1976, 1841, n.spec. =1). 


\section{Rana dalmatina}

S.I. (leg. anonim., 13-sep-1959, 570, n.spec. =4); 20-oct-1994, 631); s.d., 293); (304); 320); (326); 331); (531); 533); (569); 570); (578); 596); (599); (628); (683); 700); (713); 1309); (1359); 1369); (1371); 1417); (1433); 1484); (1512); (leg. G. Džukić s.d., 599, n.spec. = 9); (990); (1401); Dobri Do (leg. R. Šupić 13-may-1980, 1869, n.spec. =1); Ribnica (leg. Z. Stojaković 21-sep-1982, 1869, n.spec. = 1); Bosnia and Herzegovina Gacko: Gatačko Polje (leg. G. Džukić, 14-jun-1972, 699, n.spec. =1); 14-jun-1975, 654); Vrbica mine (leg. G. Džukić 13-jun-1975, 1372, n.spec. =1); Mt. Jahorina: Gola Jahorina (leg. G. Džukić 20-aug-1973, 2686, n.spec. =1); Mt. Zelengora: Tjentište (v.) (leg. G. Džukić 13-may-1983, 694/1, n.spec. = 1); Sarajevo: Kijevo (v.), Železnica river (leg. anonim. 05dec-1983, 694/1, n.spec. = 1); Croatia Baranja: Batina (v.) (leg. anonim., 08-apr-1972, 533, n.spec. = 3); Haljevo (leg. J. Mikuška 04-may-1971, 669 - 2, n.spec. =1); Tikveš (v.) (leg. J. Mikuška 26-aug1970, 669 - 2, n.spec. =9); Ilok (leg. G. Džukić 15-apr-1981, 551, n.spec. = 1); Istra: Motovun, Motovunska Šuma forest - Istarske Toplice spa (leg. M. Kalezić, E. Kletečki et al. 31-mar-1991, 2488, n.spec. = 1); Koprivnica: Peteranec (v.) (leg. anonim. 05-apr-1988, 715, n.spec. =6); Mt. Velebit (leg. anonim. 1955, 1313, n.spec. = 1); Ravni Kotari: Islam Latinski (v.), Baštica (leg. M. Kalezić 11-apr-1989, 2747, n.spec. = 1); Jarug river (leg. E. Kletečki 11-apr-1989, 1620, n.spec. = 1); Nin (v.), Vrsi (leg. E. Kletečki 11-apr-1989, 1615, n.spec. =2); Posedarje (v.) (leg. G. Džukić 11-jun-1975, 1403, n.spec. = 2); Ždralovac (leg. N. Tvrtković 11-jun-1977, 1463, n.spec. = 1); Velika Gorica: Peščenica (v.) (leg. G. Džukić 28-feb-1974, 2686, n.spec. =2); 11-jun-1976, 1399); (leg. N. Tvrtković 24-mar-1977, 1463, n.spec. = 1); Turopolje, Orle (v.) (leg. G. Džukić 23-apr-1981, 1434, n.spec. =1); (1850); Peščenica (v.) (leg. N. Tvrtković 26-apr-1976, 722, n.spec. = 6); Vukomeričke Gorice, Dragonožec (v.), Trpuci (leg. N. Tvrtković 18-apr-1976, 707, n.spec. =1); Zagreb (leg. D. Pelić, G. Džukić 15-apr-1975, 693, n.spec. = 1); Dubrava (v.) (leg. G. Džukić 01-jun-1976, 1399, n.spec. = 12); 30-jun-1976, 550); Macedonia Mt. Galičica: Mala Lokva (leg. G. Džukić, 08-may-1997, 2688, n.spec. = 1); Mt. Jablanica: Gornja Belica (v.) (leg. G. Džukić 23-sep-1996, 552, n.spec. = 3); Višni (v.) (leg. G. Džukić 23-sep-1996, 2287, n.spec. = 8); Mt. Skopska Crna Gora: Korblić (leg. E. Dauti 23-oct-1977, 1869, n.spec. = 1); Prespa lake: Ezerani (v.) (leg. anonim. 07-may-1997, 291, n.spec. =4); Struga: Belčišta (v.) (leg. G. Džukić 06-may-1997, 2668, n.spec. = 8); Montenegro Berane [“Ivangrad"] (leg. R. Celić, 13-jul-1980, 1869, n.spec. =1); (leg. V. Rodičević 17-sep-1979, 1869, n.spec. = 1); Petnjica (v.) (leg. Ć. Muratović 16-apr-1981, 1869, n.spec. = 2); Cetinje: Bjeloši (v.), Lašor puddle (leg. G. Džukić, M. Kalezić 30-may-1990, 720, n.spec. =1); Grahovo: Stena (leg. G. Džukić 25-aug-1982, 1338, n.spec. = 1); Kostiće (v.) (leg. M. Vesković 01-aug-1982, 1869, n.spec. = 4); Mt. Bjelasica: Biogradska Gora (leg. Lj. Tomović, R. Ajtić 22-jul-2000, 1830, n.spec. = 1); Biogradska Rijeka river delta (leg. J. Crnobrnja-Isailović 09-jul-1996, 2093, n.spec. = 2); mountain lodge (leg. J. Crnobrnja-Isailović, G. Džukić 13-jul-1996, 2097, n.spec. = 3); Mt. Durmitor (leg. anonim. s.d., 682, n.spec. =11); (1314); Bukovica (leg. P. Jakšić 04-jul-1987, 1309, n.spec. = 1); Poljane (leg. anonim. 06-jul-1971, 1630, n.spec. =1); Tepca (v.) (leg. anonim. 14-sep-1990, 1322, n.spec. = 2); Mt. Sinjavina: Zminičko lake (leg. G. Džukić, M. Kalezić 11-jun-2005, 2516, n.spec. =1); Mt. Žijovo: Bukumirsko lake (leg. G. Džukić, M. Kalezić 28-may-2003, 2316, n.spec. =1); Nikšić: Kruševice (leg. S. Brajušković 05-aug-1976, 1869, n.spec. = 3); Plav (leg. S. Manojlović 17-jul-1976, 1869, n.spec. = 2); Ali-Pašin Bunar (leg. G. Pasuljević 22-aug-1980, 1869, n.spec. = 1); Brezojevica (v.) (leg. G. Pasuljević 01-aug-1974, 1869, n.spec. = 1); Ulcinj: Štoj (v.) (leg. G. Pasuljević 23-jun-1976, 1869, n.spec. = 1); Velika Plaža beach (leg. G. Džukić, M. Kalezić, M. Denoel 08-jun-2005, 1382, n.spec. = 1); Serbia (leg. G. Džukić, s.d., 1269, n.spec. = 1); Aleksandrovac: Gornja Zleginja (v.), Krujavac (leg. D. Rajković 10-jun-1977, 1869, n.spec. =1); Bajina Bašta: Perućac (leg. G. Džukić 14jun-1969, 1269, n.spec. =1); Bela Crkva: Dragića Hat, Đavolji Most (leg. G. Džukić, M. Kalezić 19mar-2001, 2324, n.spec. =1); Dupljaja (v.) (leg. M. Kalezić, G. Džukić, M. Marković 19-mar-2001, 1234, n.spec. = 1); Jaruga stream (leg. G. Džukić, M. Kalezić 29-mar-2003, 1047, n.spec. =1); Mrtva Nera (leg. G. Džukić, M. Kalezić 18-mar-2001, 2147, n.spec. = 1); Jasenovo (v.), Jasenovačke Šume forests (leg. M. Kalezić, G. Džukić 22-aug-1998, 2026, n.spec. = 11); (2032); Kaluđerovo (v.) (leg. anonim. 05-apr-2012, 1320, n.spec. =1); Leskovački Potok stream (leg. G. Džukić, M. Kalezić 14-mar1999, 2047, n.spec. = 18); Kruščica (v.) (leg. G. Džukić, M. Kalezić 18-mar-2001, 1235, n.spec. = 1); (2048); Kusić (v.) (leg. G. Džukić, M. Kalezić 01-nov-2002, 677, n.spec. = 3); Mrtva Nera (leg. G. 
Džukić, M. Kalezić 05-apr-2003, 2003, n.spec. = 3); (leg. M. Kalezić, G. Džukić, M. Marković 18-mar-2005, 1077, n.spec. =1); King Alexander forest (leg. M. Kalezić, G. Džukić, M. Marković 28-mar-2004, 1245, n.spec. = 3); Nera river (leg. G. Džukić, M. Kalezić 21-apr-2000, 1062, n.spec. = 1); Orešac (v.), Straža (v.) (leg. M. Kalezić, G. Džukić, M. Marković 21-may-1997, 2110, n.spec. = 3); Beočin: Beočinske Livade (leg. anonim. 17-mar-1975, 628, n.spec. =1); Beograd: Krnjača (v.) (leg. M. Kalezić, G. Džukić 13-apr-2000, 2056, n.spec. = 2); 02-may-2000, 2064); Veliko Selo (v.), Ada Štefanac (ins.) (leg. P. Cakić 14-nov-1995, 2055, n.spec. = 1); Brodarevo: Vrbnica (v.) (leg. G. Džukić 07-jul-1969, 1269, n.spec. = 1); Deliblato sands: Deliblato (v.) - Mramorak (v.) (leg. G. Džukić, M. Kalezić 18-mar-2005, 1083, n.spec. = 5); Đurica, Stevanove Ravnice (leg. G. Džukić, M. Kalezić 21-aug-1998, 1397, n.spec. = 1); (leg. M. Kalezić 10-may-2003, 2317, n.spec. = 1); (leg. M. Kalezić, G. Džukić 20-oct-1998, 2057, n.spec. =2); Tuzla (leg. M. Kalezić, G. Džukić, M. Marković 20-may1998, 2075, n.spec. = 3); Kajtasovo (v.) (leg. G. Džukić, M. Kalezić 18-mar-2001, 1186, n.spec. =1); Majur Bara (leg. anonim. 16-may-1976, 628, n.spec. =1); (leg. G. Džukić, M. Marković 18-may-1996, 2082, n.spec. =1); Mali Pesak (leg. G. Džukić, M. Kalezić 16-jul-1998, 2089, n.spec. =1); 20-oct-1998, 576); Orlova Umka (leg. G. Džukić 18-sep-1999, 660, n.spec. = 1); Mramorak (v.), Kraljevac, Obzovik Bara, Vodice churches (leg. G. Džukić, M. Kalezić 18-mar-2005, 2217, n.spec. = 1); Šušara (v.) (leg. J. Ham 07-jul-1980, 2709, n.spec. = 3); Zamfir Bara (leg. G. Džukić, M. Kalezić 22-mar-2000, 2049, n.spec. =6); 04-jun-2000, 1097); Despotovac: Vražja Bara (leg. G. Džukić, M. Kalezić 28-feb1997, 2805, n.spec. = 1); 30-jul-1997, 1654); 12-may-2002, 1243); (leg. G. Džukić, M. Kalezić, J. Radojičić 11-mar-1997, 2076, n.spec. = 11); Gnjilane: Kosovska Kamenica, Ograđe (leg. B. Milošević 01-jul1978, 1869, n.spec. = 3); 01-aug-1979, 1869); Sveti Jovan (leg. B. Milošević 20-aug-1977, 1869, n.spec. = 3); Grdelička gorge: Predejane (v.), Džep (v.), Džepska river (leg. G. Džukić, M. Kalezić 19apr-2007, 2190, n.spec. = 4); Kladovo: Lolića Salaš (leg. G. Džukić, M. Kalezić 17-may-2000, 1048, n.spec. = 1); Zamna river (leg. G. Džukić, M. Kalezić 22-jun-2003, 1045, n.spec. = 1); 28-sep-2005, 2277); Kosovo (leg. G. Pasuljević s.d., 1869, n.spec. = 11); Kosovska Mitrovica: Banjska (v.), Jovanov Potok stream (leg. D. Todorović 14-nov-1977, 1869, n.spec. = 2); Suvi Do (v.) (leg. D. Todorović 28-may-1978, 1869, n.spec. = 2); Crni Vril (leg. D. Baun? 08-jun-1978, 1869, n.spec. =1); Leposavić, Zabrđski Potok stream (leg. G. Pasuljević 23-may-1975, 1869, n.spec. =1); Srbovac (v.) (leg. D. Todorović 26-may-1979, 1869, n.spec. =1); Zubin Potok, Lučka river (leg. S. Bašanović 30-sep-1976, 1869, n.spec. = 1); Kovin (leg. G. Džukić 05-apr-1971, 1526, n.spec. = 2); Bavanište (v.) (leg. M. Živković 01-nov-1989, 2050, n.spec. = 1); Kraljevo: Ratina (v.) (leg. G. Džukić 27-sep1980, 599, n.spec. = 1); Žiča (v.) (leg. S. Grujić 20-sep-1982, 1869, n.spec. = 1); Kučevo: Železnik (v.) (leg. V. Kalafatić 26-may-1980, 2222, n.spec. = 1); Kuršumlija: Prolom Banja spa (leg. G. Pasuljević 11-jun-1979, 1869, n.spec. = 1); Leskovac: Belanovac (v.), Pašina Česma (leg. G. Džukić, M. Kalezić 01-sep-2000, 1246, n.spec. = 2); Miroševec (leg. anonim. s.d., 570, n.spec. = 1); Vučje, Manetove Livade (leg. G. Džukić, M. Kalezić 01-sep-2000, 1106, n.spec. = 1); Loznica: Lešnica (v.) (leg. anonim. 23-mar-1991, 1528, n.spec. =2); Majdanpek: Blagojev Kamen (v.) (leg. G. Džukić 01-jun-1972, 1269, n.spec. = 3); Debeli Lug (v.), Todorova river (leg. G. Džukić, M. Kalezić 27-apr-2009, 1058, n.spec. =1); Gornjane (v.), Ljubova river (leg. K. Bajić 06-jul-1990, 2690, n.spec. = 1); Rudna Glava (v.), Lozovica stream (leg. K. Bajić 09-jul-1990, 2690, n.spec. =1); Mt. Avala: Trešnja (leg. anonim. s.d., 1242, n.spec. =1); (leg. S. Litvinchuk 22-aug-1996, 1160, n.spec. = 1); Mt. Beljanica (leg. SR i SJ? 17-jul-2004, 1831, n.spec. = 2); Mt. Fruška Gora: Andrevlje (leg. G. Džukić 01-may-1975, 1269, n.spec. =1); 12-may-1975, 1269); Osovlje (leg. G. Džukić 21-mar-1980, 599, n.spec. = 3); Testera (leg. G. Džukić 20-mar-1976, 595, n.spec. = 12); Vrdnik (v.), Vrdnička Kula (leg. G. Džukić 19-mar-1975, 1269, n.spec. = 2); Zmajevac (leg. G. Džukić 12-jul-1974, 1269, n.spec. = 1); 22-may-1975, 1269); Mt. Kopaonik: Jošanička Banja spa (leg. J. Crnobrnja, O. Glišić 30-apr-1986, 599, n.spec. = 2); Samokovska river gorge (leg. J. Crnobrnja 07-jun-1985, 599, n.spec. =1); Mt. Kukavica (leg. anonim. 10-sep-1979, 570, n.spec. = 1); Mt. Maljen: Divčibare (leg. M. Kalezić 13-mar-1994, 2123, n.spec. =2); Mt. Miroč: Cvetanovačka Bara (leg. G. Džukić, M. Kalezić 17-may-2000, 1202, n.spec. = 1); Stranjičel (leg. G. Džukić, M. Kalezić 14-may-2002, 1241, n.spec. =1); Mt. Ostrozub: Ruplje (v.) (leg. G. Džukić, M. Kalezić 12-sep-2006, 2296, n.spec. =1); Mt. Povlen: Srednji Povlen (leg. anonim. 29-jun-1999, 1240, n.spec. =2); Mt. Radan: Velika Loparda (leg. G. Džukić 21-may-1977, 1269, n.spec. = 1); Mt. Skopska Crna Gora: Letnica (v.) (leg. G. Pasuljević 01-aug-1979, 1869, n.spec. =2); Mt. Stara Planina: Vrelo (v.) (leg. G. Džukić, M. Kalezić 25-sep- 
2008, 1053, n.spec. = 1); Mt. Suva Planina: Rakoš (leg. G. Džukić, M. Kalezić, D. Jović 27-sep-2005, 1088, n.spec. = 7); Mt. Tara: Jagoštica (v.), Galine hamlet (leg. G. Džukić 21-may-1972, 1269, n.spec. = 1); Zaovine (v.), Beli Rzav river (leg. anonim. 20-may-1972, 1269, n.spec. = 1); Mt. Vršačke Planine (leg. anonim. 23-apr-1992, 536, n.spec. =1); 09-jun-2002, 1824); (leg. G. Džukić 17may-1976, 1269, n.spec. = 5); (leg. G. Džukić, M. Kalezić 23-apr-1992, 1162, n.spec. = 1); Malo Središte (v.) (leg. G. Džukić, M. Kalezić 19-may-2003, 1248, n.spec. =1); Mesić (v.) (leg. M. Kalezić, G. Džukić, M. Marković 22-may-1998, 2125, n.spec. = 1); Vršačke Čuke (leg. G. Džukić 01-jun-1970, 1526, n.spec. = 2); Vršišor (leg. G. Džukić, M. Kalezić 10-jun-2004, 1244, n.spec. = 1); Mt. Zlatar: Aljinovići (v.) (leg. J. Crnobrnja-Isailović 16-aug-1996, 2052, n.spec. =1); Mts Šar-Planina: Brezovica (v.), above (leg. P. Jakšić 05-jun-1971, 2531, n.spec. =1); Niš: Mezgraja (v.) (leg. Z. Nikolić 08-oct-1977, 1869, n.spec. = 2); Nova Varoš: Kokin Brod (v.), Uvačko lake, dam (leg. J. Crnobrnja-Isailović 18-aug-1991, 2051, n.spec. =1); Obedska Bara (leg. G. Džukić 17-apr-1970, 1526, n.spec. = 7); Obrenovac: Mali Borak (v.), Kolubara river (leg. M. Milenković 14-apr-1990, 2084, n.spec. = 1); Skela (v.) (leg. M. Milenković 08-nov-2001, 2058, n.spec. = 1); Pančevo: Gradska Šuma forest (leg. M. Kalezić, G. Džukić 20-mar-2002, 2073, n.spec. = 2); Ivanovo (v.), Danube Embankment (leg. G. Džukić, M. Kalezić 18-apr-2002, 1237, n.spec. =1); Pančevački Rit (leg. B. Žiljak 19-oct-1995, 2063, n.spec. = 2); Peć (leg. V. Sabović 20-may-1979, 1869, n.spec. =1); Đurakovac (v.), Osojane (v.) (leg. D. Lakušić 05-oct-1978, 1869, n.spec. =1); 23-may-1978, 1869); Istok (v.) (leg. G. Pasuljević 01-nov-1979, 1869, n.spec. =1); Vitomirica (v.) (leg. H. Babačić 10-jul-1978, 1869, n.spec. = 1); Zobijak (leg. H. Babačić 08-sep-1978, 1869, n.spec. = 2); Pešter Plateau: Delimeđe (v.), Stanovi, Karaiska Bara (leg. G. Džukić, M. Kalezić 28-apr-2010, 2683, n.spec. = 1); Pirot: Dojkinci (v.) (leg. G. Džukić, M. Kalezić 22-jul-2004, 1236, n.spec. = 1); Jerma river gorge (leg. J. Crnobrnja-Isailović, I. Krizmanić 01-jun-1998, 2109, n.spec. =1); Vlasi (v.), Anina Bara (leg. M. Kalezić 21-mar-2012, 989, n.spec. = 1); Krupac (v.), Krupačko Blato lake (leg. G. Džukić 22-may-1977, 1269, n.spec. =2); (leg. G. Džukić, M. Kalezić 14-may-2004, 1090, n.spec. =1); Rsovci (v.), Sveti Ilija monastery (leg. G. Džukić, M. Kalezić 25-sep-2008, 1053, n.spec. =1); wellheads (leg. G. Džukić 14may-1969, 1269, n.spec. = 2); Priština: Gračanička river (leg. G. Pasuljević 01-may-1975, 1869, n.spec. = 1); Kosovo Polje (leg. Z. Martinović 25-jun-1976, 1869, n.spec. =1); Mt. Grmija (leg. B. Bajčetić s.d., 1869, n.spec. = 2); (leg. G. Pasuljević 09-may-1970, 1869, n.spec. =1); Tank Bašti (leg. Z. Krivošej 10-jun-1981, 1869, n.spec. = 1); Velika Slatina (v.) (leg. G. Pasuljević, B. Milošević 02-jul1980, 1869, n.spec. = 1); Prizren: Dušanovo (v.), Pašina Česma (leg. G. Džukić, M. Kalezić 01-sep2000, 2045, n.spec. =15); Prokuplje (leg. anonim. 16-aug-1961, 551, n.spec. =2); (leg. M. Petković 20apr-1981, 1869, n.spec. = 1); Gornja Konjuša (v.) (leg. G. Gagulić 13-sep-1975, 1869, n.spec. = 3); Raška (leg. S. Novičević 01-jun-1977, 1869, n.spec. = 1); Sombor: Bački Monoštor (v.) (leg. Đ. M. 16-mar-1997, 2119, n.spec. = 8); Sremska Mitrovica: Zasavica (v.), Vrbovac and Pačija Bara (leg. G. Džukić, M. Kalezić 24-apr-1998, 1265, n.spec. = 1); Sremski Karlovci: Karlovački Vinogradi station (leg. A. Ivanović 25-apr-1995, 2065, n.spec. = 3); Stari Vlah: Sjenica, Uvac river, Sjenička cave (leg. anonim. 10-aug-1982, 1621, n.spec. =1); Subotica: Hrastovača, Makova Sedmica (v.) (leg. G. Džukić, M. Kalezić 08-jul-1999, 1169, n.spec. =3); Legrad (leg. G. Džukić 06-apr1988, 1029, n.spec. = 2); Makova Sedmica (v.), Hrastovača (leg. G. Džukić, M. Kalezić 13-mar1997, 2069, n.spec. = 4); (leg. M. Kalezić, G. Džukić 02-oct-1998, 2039, n.spec. =1); Hrastovača, Čavolj (leg. G. Džukić, M. Kalezić 08-mar-1998, 2079, n.spec. =1); 15-oct-1999, 2066); Subotičko-Horgoška sands, Carska Žila (leg. G. Džukić, M. Kalezić 22-mar-1996, 1333, n.spec. = 35); Surdulica: Jelašnica river gorge (leg. G. Džukić 12-jul-1978, 1464, n.spec. =1); Svilajnac: Markovac (v.), Morava river (leg. G. Džukić 14-sep-1977, 1269, n.spec. =1); Svrljig: Niševac (v.), Svrljiški Timok river (leg. J.J. Purger 19-jul-1989, 596, n.spec. =1); Šabac: Orid (v.) (leg. G. Džukić, M. Kalezić 27-apr1998, 2081, n.spec. = 1); Trstenik: Donja Crnišava (v.) (leg. M. Kalezić, G. Džukić 19-oct-1997, 2070, n.spec. = 1); Osaonica (v.) (leg. anonim. 08-jun-1977, 1869, n.spec. = 1); Tutin: gorge above (leg. N. Kokotović 13-nov-1980, 2706, n.spec. =1); Ribariće (v.) (leg. B. Prelević 10-jun-1978, 1869, n.spec. = 1); Ub: Novaci (v.), Tamnava river (leg. M. Marković 06-oct-2004, 1036, n.spec. = 1); Uroševac (leg. anonim. 07-aug-1976, 1869, n.spec. =6); (leg. R. Savić 28-may-1979, 1869, n.spec. = 2); (leg. S. Novičević 12-nov-1976, 1869, n.spec. = 11); Nerodimlje (v.) (leg. D. Masilović 14-jun-1980, 1869, n.spec. =1); Valjevo: Gola Glava (v.) (leg. G. Džukić, M. Kalezić 26-apr-1998, 2130, n.spec. = 1); Gradac river gorge (leg. G. Džukić 21-jun-1983, 1321, n.spec. = 2); Mionica, Paštrić (v.) (leg. 
anonim. 15-mar-1980, 570, n.spec. $=1$ ); Vlasinsko lake: near dam (leg. K. Ljubisavljević 22-aug1998, 2060, n.spec. = 1); Potočić (leg. K. Ljubisavljević 23-aug-1998, 2054, n.spec. =1); Vranje: Markovo Kale (leg. G. Džukić 13-may-1980, 647, n.spec. = 1); Zlot: Lazareva river canyon, Zlotska cave (leg. anonim. 14-may-1970, 628, n.spec. =1); Slovenia Ajdovščina: Štanjel (v.), Kobjeglava (leg. B. Kryštufek, 07-may-1986, 714, n.spec. $=1$ ); Dragonja river valley: Pršuti (v.) (leg. B. Kryštufek 07-may-1986, 714, n.spec. = 1); Kostanjevica: Krakovski Gozd (leg. J. Gregori 28mar-1974, 1338, n.spec. = 2); Ljubljana: Ig (v.), vicinity (leg. G. Džukić 01-apr-1979, 1820, n.spec. = 1); Mt. Šmarna Gora (leg. G. Džukić, B. Krištufek 21-may-1977, 1487, n.spec. =1); Murska Sobota: Radenci (v.), Turjanci (v.) (leg. anonim. 01-apr-1988, 1398, n.spec. =1); Nova Gorica: Panovec (v.) (leg. anonim. 16-apr-1967, 1338, n.spec. =1); Pobjeglava: Štanjel (v.) (leg. G. Džukić, M. Kalezić 03-apr-1988, 2602, n.spec. $=2$ ).

\section{Rana graeca}

S.I. (leg. anonim., 20-oct-1994, 631, n.spec. = 1); s.d., 293); (320); 539); (578); 695); (1458); 1521); (1871); (leg. J. Crnobrnja-Isailović 02-apr-2000, 2111, n.spec. = 5); Bosnia and Herzegovina Sarajevo: Kijevo (v.), Železnica river (leg. G. Džukić, 12-may-1983, 694/1, n.spec. = 3); Zvornik: Vlasenica, Tišća river wellhead (leg. anonim. 23-jul-1970, 1885, n.spec. = 1); Macedonia Berovo: Berovsko lake, Ratevska river (leg. anonim., 26-may-1970, 1885, n.spec. =6); Demir Kapija: Iberlijska gorge (leg. P. Jakšić 09-jun-1991, 1305, n.spec. =4); Mavrovo (leg. G. Mesaroš 18-jun-1987, 289, n.spec. = 1); Mt. Osogovske Planine: Ponikva (leg. G. Džukić, S. Petkovski 23-jun-1995, 215, n.spec. =1); Mt. Skopska Crna Gora: Matejče monastery (leg. BK 05-jun-1989, 1855, n.spec. = 2); Strumica: Strumičko Pole (leg. M. Kalezić, B. Kryštufek 20-apr-1990, 2528 , n.spec. =1); Montenegro (leg. anonim., s.d., 682, n.spec. = 2); Berane [“Ivangrad”] (leg. $v$. Radičević 13-sep-1979, 564, n.spec. = 1); Trpeška Rijeka stream (leg. I. Skenderović 20-aug-1987, 700 , n.spec. =2); Danilovgrad: Zagarač (v.) (leg. M. Kalezić, G. Džukić, J. Crnobrnja-Isailović, I. Aleksić 24-may-1996, 2122, n.spec. = 1); Kolašin: Morača monastery (leg. G. Džukić 08-jul-1969, 1521, n.spec. = 2); (leg. L. Vlahović 29-nov-1978, 1870, n.spec. =1); Kotor: Škudra (leg. G. Džukić 11-jul1969, 1464, n.spec. = 2); Mt. Bjelasica: Biogradska Gora (leg. G. Džukić, M. Kalezić 09-oct-1996, 2121, n.spec. =1); (leg. Lj. Tomović, R. Ajtić 22-jul-2000, 1830, n.spec. = 3); Biogradsko lake (leg. G. Pasuljević 16-jun-1970, 1870, n.spec. = 1); (leg. J. Crnobrnja-Isailović 08-jul-1996, 2124, n.spec. $=1$ ); Jezerštica (leg. G. Džukić, J. Crnobrnja-Isailović, I. Aleksić 27-jul-1997, 2036, n.spec. =6); Jezerštica (leg. J. Crnobrnja-Isailović 09-jul-1996, 2071, n.spec. = 3); Mt. Durmitor (leg. anonim. s.d., 1310, n.spec. = 5); (1314); 1630); Crepulj Poljana (leg. P. Jakšić 11-jul-1987, 1309, n.spec. = 1); Crno lake, Čeline (leg. P. Jakšić 02-jul-1987, 1309, n.spec. = 1); Svrablje lake (leg. anonim. 11-jul-1971, 1630, n.spec. = 1); Mt. Rumija: Sutorman (v.) (leg. A. Ivanović, I. Aleksić, M. Kalezić 29-may-1995, 2092, n.spec. =4); Lera (leg. G. Džukić, M. Kalezić 02-jun-1990, 1523, n.spec. =1); Mt. Sinjavina: Zaboj (v.), Zabojsko lake (leg. G. Džukić, M. Kalezić 21-aug-2002, 1274, n.spec. =14); Mts Prokletije: Rikavačko lake (leg. G. Džukić, M. Kalezić, A. Ivanović 20-sep-1991, 1839, n.spec. = 20); 20-sep-1991, 1839); Plav (leg. Z.B. 17-jul-1976, 1870, n.spec. =2); Podgorica: Cijevna river (leg. K. Ljubisavljević 03-sep-1999, 2074, n.spec. = 4); Lijeva Rijeka (v.), Jablan (v.) (leg. M. Kalezić, G. Džukić, M. Marković 28-may-2003, 2297, n.spec. = 1); Rijeka Crnojevića: Orahovštica stream (leg. G. Džukić 11-mar1974, 1332, n.spec. = 2); Šavnik: Komarnica canyon (leg. G. Džukić, M. Kalezić 15-apr-1992, 1302, n.spec. = 1); Pošćensko lake (leg. G. Džukić, J. Crnobrnja-Isailović, I. Aleksić 30-jul-1997, 2682, n.spec. $=1$ ); Tara river canyon: Brštanovice, NW from (leg. G. Džukić 15-sep-1990, 1525, n.spec. = 2); Prštanovica (leg. anonim. 22-jul-1988, 682, n.spec. =1); Serbia (leg. anonim., s.d., 1464, n.spec. $=$ 1); (leg. G. Pasuljević s.d., 1870, n.spec. = 20); (leg. G. Pasuljević, G. Džukić, B. Milošević 18-jul-1980, 566, n.spec. =2); Bosilegrad: Bistar (v.) (leg. G. Džukić 19-may-1978, 1464, n.spec. =1); Rajčilovci (v.) (leg. M. Kalezić 23-apr-2014, 2382, n.spec. =1); Gnjilane: Kosovska Kamenica (leg. B. Milošević 26-jul-1979, 566, n.spec. =1); Ograđe (leg. B. Milošević 01-may-1978, 1870, n.spec. = 4); 09aug-1979, 566); Sveti Jovan (leg. B. Milošević 20-aug-1977, 1870, n.spec. = 5); Grdelička gorge: Migulovac (v.) (leg. G. Džukić, M. Kalezić 18-apr-2007, 1055, n.spec. =1); Predejane (v.) (leg. G. Džukić, M. Kalezić 20-apr-2007, 2156, n.spec. = 4); Hacijenda (leg. G. Džukić, M. Kalezić 01-apr-2007, 1061, n.spec. = 2); Predejanska river (leg. G. Džukić, M. Kalezić 31-mar-2007, 2190, n.spec. = 2); 
Kosovska Mitrovica (leg. D. Todorović 26-may-1979, 566, n.spec. = 1); Banjska (v.) (leg. D. Todorović 09-nov-1977, 1870, n.spec. = 14); 24-mar-1981, 565); 28-oct-1977, 1870); Grižane (v.) (leg. D. Todorović 18-apr-1978, 1870, n.spec. = 2); Jankov Potok stream (leg. D. Todorović 14-nov-1977, 1870, n.spec. = 3); Ješevik (leg. D. Todorović 08-apr-1978, 1870, n.spec. = 8); Junake (v.) (leg. D. Todorović 07-jun-1978, 1870, n.spec. = 1); Rudnik (leg. D. Todorović 11-apr-1978, 1870, n.spec. =1); Vakač (leg. D. Todorović 04-may-1978, 1870, n.spec. =2); (leg. G. Todorović 04-may-1978, 700, n.spec. =1); Leposavić, Jošanica (v.) (leg. G. Pasuljević 03-jun-1973, 1870, n.spec. = 2); Krivaja river (leg. Ž. Tomanović 02-may-1990, 1438, n.spec. = 1); Kuršumlija: Prolom Banja spa (leg. G. Pasuljević 11jun-1979, 566, n.spec. = 2); Leskovac: Vučje, Vučjanka, Đokini Virovi (leg. G. Džukić 16-jun-2001, 1044, n.spec. = 3); Ljubovija: Gornja Trešnjica (v.) (leg. J. Crnobrnja-Isailović 23-mar-1999, 1102, n.spec. = 6); (leg. J. Crnobrnja-Isailović, I. Aleksić 23-mar-1999, 2053, n.spec. = 3); (leg. Ž. Tomanović 01jun-1990, 1438, n.spec. = 7); 17-may-1990, 1632); Trešnjica (v.) (leg. G. Džukić 27-may-1972, 1521, n.spec. = 1); Trešnjica canyon (leg. G. Džukić 12-mar-1995, 2127, n.spec. = 2); Mionica: Ribnička cave (leg. G. Džukić 15-mar-1980, 1871, n.spec. =1); Mt. Besna Kobila: Musulj (v.), Crna Reka stream (leg. M. Kalezić, G. Džukić, M. Marković 25-sep-2005, 2775, n.spec. =2); Mt. Jadovnik: Grozd (leg. J. Crnobrnja-Isailović 27-aug-1997, 2128, n.spec. =10); Mt. Jastrebac: Buci (v.) (leg. G. Džukić 17-oct-1977, 630, n.spec. = 1); (1464); Mt. Kopaonik (leg. J. Crnobrnja-Isailović 04-jun-1985, 1871, n.spec. = 1); Jošanička Banja spa (leg. O. Glišić 30-apr-1986, 1871, n.spec. =1); Samokovska river gorge (leg. J. Crnobrnja-Isailović 07-jun-1985, 1871, n.spec. =1); Mt. Maljen: Divčibare (leg. M. Kalezić 13-mar-1994, 2135, n.spec. = 1); 24-jun-1995, 2046); Crna Kamenica river (leg. J. Crnobrnja-Isailović 25-jul-1999, 2027, n.spec. = 3); Serđova Bara (leg. J. Crnobrnja-Isailović 24-jul-1999, 2100, n.spec. = 2); Mt. Medvednik: Stave (v.) (leg. M. Kalezić 27-aug-2004, 2022, n.spec. =1); Mt. Prolomska Planina: Zebice (v.), Đavolja Varoš (leg. anonim. 30-aug-2005, 1072, n.spec. = 3); Mt. Radan: Gajtan (v.) (leg. G. Džukić 21-may-1977, 1464, n.spec. = 1); Mt. Skopska Crna Gora: Letnica (v.) (leg. G. Pasuljević 08-aug-1979, 562, n.spec. = 8); Mt. Tara (leg. G. Džukić 09-jun-1969, 1464, n.spec. = 3); Jagoštica (v.), Galine hamlet (leg. G. Džukić 01-may-1972, 1464, n.spec. = 1); Mitrovac (v.), Crveni Potok stream (leg. G. Džukić 15-aug-1882, 1682, n.spec. = 1); Mt. Zlatar: Aljinovići (v.) (leg. J. Crnobrnja-Isailović 16-aug-1996, 2052, n.spec. =1); Mts Šar-Planina: Brezovica (v.) (leg. G. Pasuljević 4-jun-1971, 1870, n.spec. = 2); (leg. P. Jakšić 11-jun-1988, 702, n.spec. = 1); (leg. P. Stamenković 02-sep-1976, 1869, n.spec. =1); Careva Livada (leg. G. Pasuljević 11-oct1977, 1870, n.spec. = 1); Dragaš, Rapča (v.) (leg. M. Hajredini 22-mar-1980, 566, n.spec. = 2); Mt. Ljuboten (leg. G. Džukić 06-jul-1977, 1464, n.spec. =1); Nova Varoš: Kokin Brod (v.), Neškoviće (v.), Šupljica (leg. G. Džukić 11-aug-1982, 1464, n.spec. = 2); Uvac river (leg. anonim. 19-may-1988, 697, n.spec. = 1); Uvac river canyon (leg. J. Crnobrnja-Isailović 29-aug-1997, 2126, n.spec. =1); Novi Pazar (leg. D. Todorović 24-may-1978, 1870, n.spec. =1); Peć: Beli Drim spring (leg. G. Pasuljević 01-jun-1979, 566, n.spec. = 1); Dečane, Dečanska Bistrica gorge (leg. V. Stevanović 12-jul-1977, 1464, n.spec. =2); Istok (v.) (leg. G. Pasuljević 25-nov-1970, 1870, n.spec. =1); Radavac (v.) (leg. G. Džukić 07-jun-1977, 1464, n.spec. = 1); Peć - Đakovica: Juničko Polje (leg. G. Džukić 05-jun1977, 1464, n.spec. = 1); Pirot: Zvonce (v.) (leg. J. Crnobrnja-Isailović, I. Aleksić 02-jun-1998, 308, n.spec. =1); Priboj (leg. Z. Kljajić 15-nov-1979, 566, n.spec. =1); Kratovska river (leg. G. Džukić 12aug-1982, 1464, n.spec. = 1); Prijepolje: Staro (v.) (leg. G. Džukić 06-jul-1979, 1464, n.spec. = 1); Prizren: Sredska (v.) (leg. I. Ivić 05-nov-1976, 1870, n.spec. =1); Stari Vlah: Sjenica, Čedovo (v.) (leg. D. Mijajlović 10-jun-1976, 1870, n.spec. = 8); Uvac river, Sjenička cave (leg. anonim. 10-aug1982, 1621, n.spec. = 1); Surdulica: Jelašnica river gorge (leg. G. Džukić 12-jul-1978, 1464, n.spec. = 8); Suva Reka: Dulje (v.), Crnoljevo (v.) (leg. G. Pasuljević, G. Džukić, B. Milošević 06-jul1979, 566, n.spec. = 3); Matos gorge (leg. G. Džukić 05-jul-1979, 1464, n.spec. = 2); Takovo: Beršići (v.) (leg. D. Kataranovski 15-jul-1993, 1380, n.spec. = 2); Dičina river (leg. anonim. 12-aug1992, 1844, n.spec. = 2); 14-aug-1988, 2209); Savinac (v.) - Drenova river (leg. D. Kataranovski 03-aug-1998, 1514, n.spec. = 1); Trstenik: Donja Crnišava (v.) (leg. K. Ljubisavljević 27-jul-1998, 2077, n.spec. = 2); 23-may-1998, 2043); 24-may-1998, 2068); Užička Požega: Mađer (v.) (leg. M. Marković 03-may-1987, 1887, n.spec. = 6); Valjevo: Gradac river, Suvaja (leg. A. Hegediš 28-may1992, 540, n.spec. = 2); Valjevska Kamenica (v.) (leg. M. Kalezić 30-apr-1990, 1635, n.spec. = 1); Vučitrn: Samodraža (v.) (leg. S.J. 26-aug-1976, 1870, n.spec. = 5). 


\section{Rana temporaria}

S.l. (leg. anonim., 17-jul-1964, 1328, n.spec. = 2); 20-oct-1994, 631); s.d., 293); (304); 305); (321); 330); (331); 539); (578); 599); (630); 632); (695); 698); (1515); 2751); (leg. G. Džukić s.d., 698, n.spec. = 1); (leg. G. Pasuljević s.d., 653, n.spec. = 16); Bosnia and Herzegovina Mt. Jahorina (leg. anonim., 01-may-1905, 730, n.spec. = 2); Zvornik: Vlasenica, Tišća river wellhead (leg. anonim. 23-jul-1970, 1885, n.spec. = 3); Croatia Sisak: Odra river (leg. N. Tvrtković, 23-mar-1977, 1463, n.spec. =1); Varaždin: Donja Voća (v.) (leg. B. Jalžić 18-jul-1976, 1343, n.spec. =1); Velika Gorica: Peščenica (v.) (leg. N. Tvrtković 24-mar-1977, 710, n.spec. =1); Zagreb: Maksimir (leg. N. Tvrtković 27-mar-1976, 724, n.spec. = 3); Macedonia Berovo: Berovsko lake, Ratevska river (leg. G. Džukić, 26-may-1970, 710, n.spec. =1); Mt. Jablanica: Podgorečko lake (leg. G. Džukić, R.S. Petkovski 21-sep-1996, 2285, n.spec. =4); Mt. Korab: Ćos Alija (leg. G. Džukić, M. Vehbi 18-sep1996, 2280, n.spec. = 2); Kobilino Polje (leg. G. Džukić 16-sep-1996, 2283, n.spec. = 2); Mt. Osogovske Planine: Ponikva (leg. G. Džukić, S. Petkovski 23-jun-1995, 215, n.spec. = 3); Mts ŠarPlanina: Rudoka, Smreka river (leg. G. Džukić 19-jul-1978, 1437, n.spec. =2); Vakaf, Kajnak (leg. G. Džukić 18-jul-1978, 1437, n.spec. = 2); Montenegro Berane [“Ivangrad"] (leg. M. Račić, 06sep-1979, 632, n.spec. =1); (leg. V. Radičević 13-sep-1979, 564, n.spec. = 4); Mt. Bjelasica (leg. J. Crnobrnja-Isailović, G. Džukić 13-jul-1996, 2097, n.spec. = 3); Biogradska Gora (leg. Lj. Tomović, R. Ajtić 22-jul-2000, 1830, n.spec. = 2); Biogradska Rijeka river delta (leg. J. Crnobrnja-lsailović 09-jul1996, 2093, n.spec. = 6); Biogradsko lake (leg. G. Pasuljević 16-jun-1970, 632, n.spec. = 1); Biogradska river delta (leg. G. Džukić, J. Crnobrnja-Isailović, I. Aleksić 27-jul-1997, 2101, n.spec. = 2); Ričica lake (leg. J. Crnobrnja-Isailović 12-jul-1996, 2088, n.spec. = 13); Rupe Katun challet (leg. G. Džukić, J. Crnobrnja-Isailović, I. Aleksić 29-jul-1997, 2121, n.spec. = 5); Ursulovac lake (leg. G. Džukić, J. Crnobrnja-Isailović, I. Aleksić 28-jul-1997, 2116, n.spec. =6); Voleš, road to (leg. G. Džukić, J. CrnobrnjaIsailović, I. Aleksić 24-jul-1997, 309, n.spec. = 1); Vrtača, Ševarina lake (leg. G. Džukić, J. CrnobrnjaIsailović, I. Aleksić 26-jul-1997, 2059, n.spec. = 2); Mt. Durmitor (leg. anonim. s.d., 1400, n.spec. = 7); (1630); Crepulj Poljana (leg. P. Jakšić 11-jul-1987, 1309, n.spec. = 1); Žabljak (leg. P. Jakšić 02-jul1987, 1309, n.spec. =1); Mt. Žijovo: Bukumirsko lake (leg. anonim. 28-may-2003, 2518, n.spec. = 1); Mts Prokletije: Mt. Bogićevica, Babino Polje (leg. G. Pasuljević 10-aug-1980, 703, n.spec. =1); 20-aug-1980, 633); (1898); Ridsko lake (leg. G. Pasuljević 07-sep-1970, 703, n.spec. =2); Vusanje (v.), Savina Voda (leg. G. Džukić 09-jun-1977, 698, n.spec. = 2); Prokletije: Vusanje (v.), Ćafa Bor pass, Čelića challet, Rustemova Livada (leg. M. Lakušić, Z. Bulić 06-jul-1995, 248, n.spec. =2); Rožaj: Ibar river (leg. G. Pasuljević, A. Ago 01-jul-1974, 703, n.spec. = 1); Norway Oslo: Notodden, Lauvassaera (leg. B. Kryštufek, 01-jul-1992, 1604, n.spec. =2); Serbia (leg. anonim., s.d., 702, n.spec. =4); (1634); Bela Crkva: Jaruga stream (leg. G. Džukić, M. Marković, M. Kalezić 29mar-2003, 2011, n.spec. = 1); Crna Trava: Biljine Bare (leg. G. Džukić, M. Kalezić 23-jun-2007, 2152, n.spec. = 3); Kosovo (leg. G. Pasuljević s.d., 633, n.spec. $=8$ ); (703); Ljubovija: Gledeće (v.) (leg. J. Crnobrnja-Isailović, I. Aleksić 22-mar-1999, 689, n.spec. =1); Mali Zvornik (leg. V. Smiljanić 25-apr1980, 632, n.spec. =1); Mt. Beljanica (leg. SR i SJ? 17-jul-2004, 1831, n.spec. =2); Mt. Čemernik: Mlačište (v.) (leg. G. Džukić, M. Kalezić 31-mar-2007, 2150, n.spec. =1); Mt. Goč: Stanišinci (v.) (leg. B. Luković 28-may-1998, 2061, n.spec. =1); Mt. Golija (leg. G. Džukić 18-sep-1969, 1521, n.spec. = 1); (1634); Biser Voda (leg. anonim. 17-jun-1970, 1634, n.spec. =2); Mt. Jablanik (leg. anonim. 03jul-1999, 1192, n.spec. = 1); Bebića Luke (leg. anonim. 29-apr-1992, 1601, n.spec. =1); Mt. Jagodnja: Mačkov Kamen (leg. M. Kalezić, G. Džukić 01-jun-1997, 2062, n.spec. = 2); Mt. Kopaonik (leg. anonim. 04-jun-1985, 1634, n.spec. =2); Jablanova Ravan (leg. I. Ljutić, J. CrnobrnjaIsailović 28-may-1986, 1634, n.spec. = 2); Jankova Bara (leg. G. Džukić 20-oct-1977, 630, n.spec. = 2); Mt. Kukavica: Priver Voda (leg. G. Džukić 20-may-1977, 698, n.spec. =1); Mt. Miroč: Mt. Veliki Štrbac, Ploča (leg. G. Džukić 16-oct-1980, 599, n.spec. =1); Mt. Ostrozub: Zeleniče (leg. G. Džukić, M. Kalezić 22-jun-2007, 2155, n.spec. =2); Mt. Povlen: Madžari (v.) (leg. anonim. 27-apr1992, 1601, n.spec. =4); Mali Povlen (leg. anonim. 30-jun-1999, 1238, n.spec. =1); Pašina Ravan (leg. anonim. 29-jun-1999, 1238, n.spec. = 1); Srednji Povlen (leg. anonim. 01-jul-1999, 1192, n.spec. = 1); Mt. Stara Planina: Kopren (leg. G. Džukić, M. Kalezić 03-sep-2004, 2004, n.spec. =2); Mt. Tara (leg. G. Džukić 10-jun-1969, 630, n.spec. = 5); Crni Vrh, Kapetanova Voda (leg. G. Džukić 22-may1972, 630, n.spec. = 3); lake (leg. G. Džukić 21-oct-1973, 599, n.spec. =1); (1634); Zaovine (v.), Beli 
Rzav lake (leg. G. Džukić 20-may-1972, 698, n.spec. = 2); Mt. Zlatibor: Tusto Brdo (v.) (leg. anonim. 28-jun-1999, 1192, n.spec. = 1); Mts Prokletije: Mt. Mokra Gora, Ponor (leg. G. Džukić 12jun-1977, 698, n.spec. = 3); Mts Šar-Planina (leg. G. Pasuljević s.d., 633, n.spec. = 7); (703); Brezovica (v.), above (leg. P. Jakšić 03-jun-1971, 632, n.spec. =1); Stojkova Kuća, Durlov Potok stream (leg. P. Jakšić 18-jul-1986, 698, n.spec. = 1); Dragaš, Rapča (v.) (leg. M. Hajredini 22-mar1980, 566, n.spec. = 1); Jezerska Čuka, Gornja Šija (leg. B. Bajčetić 08-jul-1984, 1896, n.spec. = 1); Piribreg, Durlov Potok stream (leg. P. Jakšić 13-jul-1988, 702, n.spec. = 2); Restelica (v.), Popova Šapka (leg. G. Džukić 02-oct-1977, 1350, n.spec. = 1); Popova Šapka, Suva river (leg. anonim. 30-jul-1995, 2041, n.spec. = 11); (leg. G. Džukić, M. Kalezić, J. Crnobrnja-Isailović 30-jul-1995, 2096, n.spec. = 23); Stojkova Kuća (leg. G. Pasuljević 01-jun-1970, 632, n.spec. =1); Štrpce (leg. G. Pasuljević 22-may-1968, 633, n.spec. = 1); Prevalac (leg. G. Džukić 01-jul-1971, 698, n.spec. = 1); Trpeznica, Bogovinsko lake (Belo) (leg. G. Džukić 19-jul-1978, 622, n.spec. = 10); Nova Varoš: Kokin Brod (v.), Neškoviće (v.), Šupljica (leg. G. Džukić 11-aug-1982, 2703, n.spec. = 14); Pirot: Temska (v.) (leg. G. Džukić, M. Kalezić 25-sep-2008, 1053, n.spec. = 2); Stari Vlah: Sjenica, Raždaginja (v.) (leg. G. Pasuljević, H. Mešić 20-aug-1975, 703, n.spec. =1); (leg. H. Mešić 20-aug-1975, 632, n.spec. = 1); Slovenia Cerknica: Velike Bloke (v.) (leg. B. Horvat, 16-feb-1989, 1334, n.spec. =1); Ljutomer: Žerovinci (v.) (leg. G. Džukić 31-mar-1988, 723, n.spec. =7); Mt. Pohorje: Lovrenška lakes (leg. anonim. 1986, 714, n.spec. =1); (leg. B. Kryštufek 04-may-1986, 714, n.spec. = 1); Novo Mesto: Dolenjske Toplice spa, Podtur (v.) (leg. B. Horvat, I. Kočevar 18-jun-1986, 714, n.spec. = 1); Klevevž (v.) (leg. B. Kryštufek 23-apr-1986, 726, n.spec. =1); Mirna (v.), Zabrđe (leg. B. Kryštufek 23-apr-1986, 726, n.spec. =1).

\section{Ranae fuscae}

S.I. (leg. anonim., s.d., 675, n.spec. = 2); (1609); 1849); Montenegro Mt. Bjelasica (leg. J. Crnobrnja-Isailović, G. Džukić, 13-jul-1996, 2097, n.spec. = 2); Tara river canyon: Tepca (v.) (leg. anonim. 01-jul-2001, 1829, n.spec. = 3); Serbia Gnjilane (leg. D. Jović, 05-jul-1982, 1895, n.spec. = 1); Kosovska Kamenica (leg. B. Milošević 21-jul-1979, 1895, n.spec. = 3); Grdelička gorge: Predejane (v.) (leg. G. Džukić, M. Kalezić 20-apr-2007, 2156, n.spec. = 3); Mt. Koritnik (leg. M. Hajredini 15-sep-1981, 1895, n.spec. = 1); Mt. Rudnik (leg. D. Komatina 10-jun-1982, 1895, n.spec. =1); Mt. Tara (leg. anonim. 06-jul-1958, 1846, n.spec. = 29); Mts Šar-Planina: Jezerska Čuka, Gornja Šija (leg. B. Bajčetić 08-jul-1984, 1895, n.spec. = 3); Pešter Plateau: Karajukića Bunari (v.) (leg. J. Crnobrnja-Isailović 28-aug-1997, 2669, n.spec. = 8); Priboj (leg. E. Klajić 10-jun-1982, 1895, n.spec. = 1); Priština (leg. G. Pasuljević and students 13-may-1983, 1895, n.spec. =1); Kosovo Polje (leg. D. Čukić 10-aug-1980, 1895, n.spec. =1); (leg. M. Dimić 15-jun-1982, 1895, n.spec. =1); Mt. Grmija (leg. Lj. Sretić 18-may-1980, 1895, n.spec. =1); Valjevo: Pašina Ravan, Trešnjica river (leg. anonim. 26apr-1992, 1601, n.spec. $=2$ ).

\section{Caudata}

\section{PleThodontidaE}

\section{Batrachoseps attennatus}

USA Sonoma lake (leg. D. Hedgecock, M. Kalezić, 09-aug-1976, 1340, n.spec. = 5).

\section{PROTEIDAE}

\section{Proteus anguinus}

S.I. (leg. anonim., s.d., 1605, n.spec. =1); (2811); (leg. B. Bulog s.d., 1513, n.spec. =2). 


\section{SALAMANDRIDAE}

\section{Ichthyosaura alpestris}

S.I. (leg. anonim., s.d., 276, n.spec. = 2); (293); 318); (321); 327); (539); 544); (549); 695); (1308); 1309); (1326); 1346); (1485); 1516); (1610); 2175); (2178); 2198); (2236); 2242); (2639); 2645); (2751); (2761); (leg. G. Džukić s.d., 352, n.spec. =4); (353); (leg. G. Pasuljević s.d., 357, n.spec. = 1); (leg. M. Radovanović s.d., 1469, n.spec. =6); (1471); Babrov (leg. G. Džukić 20-aug-1976, 352, n.spec. = 1); Bosnia and Herzegovina Bosansko Grahovo: Korana stream, Pišteljak (leg. anonim., 09-apr-1989, 2662, n.spec. = 15); Čajniče (leg. anonim. 21-sep-1991, 1501, n.spec. = 125); Glamoč (leg. G. Džukić, M. Kalezić 18-may-1983, 2625, n.spec. =1); (2691); Kupres (leg. G. Džukić 17-may1983, 2647, n.spec. = 31); Bukavička Previja, lake (leg. G. Džukić 05-jun-1985, 2522, n.spec. =4); Kurića Poljane (leg. G. Džukić 04-jun-1985, 2640, n.spec. = 50); Rastičevsko lake (leg. G. Džukić, M. Kalezić 04-jun-1985, 2633, n.spec. = 119); Mt Treskavica: Zmijsko lake (leg. M. Radovanović 22jul-1955, 2474, n.spec. =4); 24-jul-1955, 2474); Mt. Čvrsnica (leg. anonim. 20-jun-1971, 352, n.spec. = 2); Mt. Igman (leg. G. Džukić 01-aug-1974, 352, n.spec. =1); Veliko Polje (leg. anonim. 01-jun1949, 1473, n.spec. = 4); Mt. Šator: Šatorsko lake (leg. anonim. 14-sep-1983, 726, n.spec. =4); (leg. G. Džukić 30-may-1985, 2249, n.spec. = 8); (leg. M. Radovanović 14-aug-1951, 1471, n.spec. = 5); Mt. Treskavica: Veliko lake (leg. St. Popović 10-jun-1951, 1008, n.spec. =6); Mt. Vranica: Prokoško lake (leg. anonim. 06-jul-1980, 2489, n.spec. = 35); (2490); 11-jul-1996, 1450); 01-aug-1951, 1473); (leg. G. Džukić 06-jul-1988, 2290, n.spec. = 39); (leg. M. Kalezić 1983, 2171, n.spec. = 40); 06-jul-1980, 1819); (leg. M. Radovanović s.d., 2474, n.spec. = 4); Mt. Zelengora: Gornje Bare lakes (leg. G. Džukić, M. Kalezić 12-may-1983, 2693, n.spec. =70); Tjentište (v.) (leg. G. Džukić 13-may-1983, 694/1, n.spec. = 2); Nevesinje: Nevesinjsko Polje, Majdan (leg. anonim. 15-may-1983, 2477, n.spec. = 19); Seljani (v.) (leg. anonim. 20-apr-1991, 2481, n.spec. = 49); (2643); Sopilja (v.), chasm (leg. anonim. 16-may-1983, 2270, n.spec. = 24); Spilja (leg. anonim. 16-may-1983, 2648, n.spec. = 28); Perućica: Tjentište (v.) (leg. G. Džukić, M. Kalezić 24-aug-2002, 2314, n.spec. = 7); Sarajevo: Dobro Polje (leg. G. Džukić 12-may-1983, 694/1, n.spec. =3); Teslić (leg. anonim. 12-apr-1980, 2020, n.spec. = 5); Croatia Benkovac: Bjelina (v.), Kalanji (v.) (leg. G. Džukić, M. Kalezić, 24-may-1984, 2636, n.spec. =22); Donji Lapac: Lapačka Korita (leg. G. Džukić 21-may-1987, 1482, n.spec. = 53); Gorski Kotar: Crni Lug (v.) (leg. M. Radovanović 17-jul-1964, 1479, n.spec. =2); Velika Voda (leg. M. Radovanović 21-aug-1963, 1477, n.spec. = 1); Gospić: Lički Osik (v.), Vinokop pond (leg. G. Džukić, M. Kalezić 01-jun-1980, 1822, n.spec. = 69); Smiljan (v.) (leg. G. Džukić 02-jun-1985, 2268, n.spec. = 20); Gračac: Žegar (v.), Ušljebrka (leg. anonim. 01-may-1985, 2480, n.spec. = 70); Knin: Ervenik (v.), Pajića puddle (leg. G. Džukić, M. Kalezić 31-may-1985, 2637, n.spec. =157); Kistanje, Grulovići (leg. G. Džukić, M. Kalezić 10-apr-1989, 993, n.spec. =64); Krbavsko Polje: Landonov Gaj (leg. G. Džukić, M. Kalezić 18-apr-1989, 2631, n.spec. = 55); Krk (ins.): Diviska (v.) (leg. A. Đorović 1990, 2386, n.spec. = 5); Kupres (leg. anonim. 17-may-1983, 2493, n.spec. = 78); (leg. G. Džukić, M. Kalezić 17-may-1983, 2641, n.spec. = 80); Zlo (v.) (leg. anonim. 09-aug-1951, 1472, n.spec. = 1); Mt. Dinara: Sinj, Kamešnica (leg. G. Džukić 22-may-1987, 1854, n.spec. = 7); Mt. Papuk: Jankovac (leg. anonim. 06-may-1981, 1280, n.spec. = 39); (1292); Mt. Plješevica: Kutarevo (leg. anonim. 01-apr-1979, 2487, n.spec. = 39); (2504); Mt. Velebit (leg. anonim. 1955, 1313, n.spec. =4); Obrovac (leg. G. Džukić, M. Kalezić 02-jun-1985, 2241, n.spec. =1); Ravni Golubić (v.) (leg. G. Džukić 01-jun-1985, 2649, n.spec. = 24); Otočac: Vrhovine (v.), Doljani, Lipice (leg. anonim. 18sep-1961, 1467, n.spec. =9); Donji Borik (leg. M. Radovanović 21-jul-1961, 1469, n.spec. = 3); Ravni Kotari: Urukolovac Hamlet (leg. anonim. 20-apr-1986, 2765, n.spec. = 3); Sinj: Mt. Svilaja (leg. J. Gelenčir 25-jul-1960, 352, n.spec. = 3); Varaždin: Donja Voća (v.) (leg. G. Džukić 18-jul-1976, 352, n.spec. =1); Velika Gorica: Peščenica (v.) (leg. G. Džukić 28-feb-1974, 352, n.spec. = 6); $24-$ apr-1981, 2697); Turopolje, Orle (v.) (leg. G. Džukić 20-may-1987, 1853, n.spec. = 16); Zagorje: Vukovo (v.) (leg. anonim. 08-jul-1962, 1467, n.spec. =1); Zagreb: Dubrava (v.) (leg. G. Džukić 30jun-1976, 550, n.spec. =1); Macedonia Mt. Jablanica: Podgorečko lake (leg. G. Džukić, 25-sep1996, 2492, n.spec. = 50); Mts Šar-Planina: Rudoka, Crno lake (leg. M. Radovanović 09-aug-1953, 1469, n.spec. =1); Trpeznica, Bogovinsko lake (Belo) (leg. G. Džukić 19-jul-1978, 353, n.spec. =4); Montenegro Berane [“Ivangrad"] (leg. D. Papović, 14-may-1980, 282, n.spec. =6); (leg. Lj. Sretić 18-jul-1980, 282, n.spec. =4); Bijelo Polje: Bistrica river (leg. anonim. 06-aug-1958, 1847, n.spec. = 
3); Kolašin: Uljara (leg. anonim. 06-jul-1979, 2698, n.spec. = 19); Mt Sinjavina: Zminičko lake (leg. M. Radovanović 1960, 1477, n.spec. = 3); 04-aug-1959, 1479); (leg. S. Marinković 01-aug-1977, 2760, n.spec. = 1); Mt. Bioč: Stabna (v.), Stubica (v.), Brštevac (leg. G. Džukić, M. Kalezić 06-jun2008, 2231, n.spec. = 1); Mt. Bjelasica (leg. Lj. Tomović, R. Ajtić 22-jul-2000, 1830, n.spec. = 1); Biogradsko lake (leg. G. Džukić 08-jul-1996, 1812, n.spec. = 51); Dolovi (leg. J. Crnobrnja-Isailović, G. Džukić 11-jul-1996, 2657, n.spec. = 20); Goleš Katun challet, road to (leg. G. Džukić, J. CrnobrnjaIsailović, I. Aleksić 24-jul-1997, 2664, n.spec. = 14); Katun Škale challet, Blato (leg. G. Džukić, J. Crnobrnja-Isailović, I. Aleksić 29-jul-1997, 1816, n.spec. = 40); Kurikućsko Blato (leg. M. Rančić 13-sep1979, 2246, n.spec. = 8); Kurikućsko lake (leg. M. Račić 10-sep-1979, 1466, n.spec. = 4); (leg. V. Radičević 13-sep-1979, 2651, n.spec. =9); Malo Šiško lake (leg. G. Džukić 11-jul-1996, 1811, n.spec. = 8); Morena (leg. J. Crnobrnja-isailović, G. Džukić 11-jul-1996, 2519, n.spec. =1); Pešića lake (leg. G. Džukić 12-jul-1996, 1815, n.spec. = 3); Šiško lake (leg. M. Radovanović 12-aug-1948, 2474, n.spec. = 2); Ursulovac lake (leg. anonim. 21-jul-1982, 1508, n.spec. =1); Veliko and malo Ursulovac lake (leg. J. Crnobrnja-Isailović, I. Aleksić, G. Džukić 28-jul-1997, 2656, n.spec. = 40); Veliko Šiško lake (leg. J. Crnobrnja-Isailović 12-jul-1996, 2139, n.spec. = 42); Vrtača, Ševarina lake (leg. G. Džukić, J. Crnobrnja-Isailović, I. Aleksić 24-jul-1997, 309, n.spec. =1); Mt. Durmitor (leg. anonim. s.d., 682, n.spec. = 21); (1310); 1314); (1400); (leg. G. Džukić s.d., 2665, n.spec. =66); (leg. M. Radovanović 24-jul-1947, 2474, n.spec. = 1); 26-jul-1947, 2474); Crno lake (leg. anonim. 08-jul-1979, 2698, n.spec. = 8); s.d., 1467); (leg. M. Radovanović s.d., 1468, n.spec. = 3); Crno lake - Zminje lake (leg. V. Anđelković 01jul-1958, 1861, n.spec. = 2); Donja Ališnica (leg. A. Ćetković 30-jun-1988, 2634, n.spec. = 32); Jablan lake (leg. M. Radovanović s.d., 1477, n.spec. = 1); Malo lake (leg. M. Radovanović 06-aug1959, 1473, n.spec. = 30); Malo Škrško lake (leg. M. Radovanović 07-aug-1959, 2474, n.spec. = 5); Valovito lake (leg. anonim. 11-jul-1971, 1449, n.spec. =7); Radeča Voda (leg. G. Džukić, M. Kalezić 10-jul-1977, 2635, n.spec. = 37); Vražje lake (leg. Josif Pančić BID 09-jul-1977, 2642, n.spec. = 18); (leg. V. Vasić 15-jul-1974, 352, n.spec. = 2); Zminičko lake (leg. anonim. s.d., 2655, n.spec. = 15); Zminje lake (leg. anonim. 02-aug-1988, 1041, n.spec. =12); (leg. G. Džukić 01-jul-1979, 2695, n.spec. = 104); (leg. G. Džukić, V. Vasić 20-jul-1975, 1630, n.spec. = 4); (leg. M. Kalezić 06-jul-1979, 1818, n.spec. = 43); (leg. M. Radovanović 06-aug-1959, 1473, n.spec. = 10); 21-aug-1960, 1479); Mt. Lukavica: Kapetanovo lake (leg. G. Džukić, M. Kalezić 23-aug-2002, 2491, n.spec. = 19); (2521); (leg. M. Radovanović 10-aug-1959, 1477, n.spec. = 5); (1502); 2474); Manito lake (leg. anonim. s.d., 2173, n.spec. = 40); (2540); (leg. G. Džukić, M. Kalezić 17-aug-1984, 2419, n.spec. = 143); (leg. M. Radovanović 10-aug-1959, 1471, n.spec. =10); Mt. Maglić: Trnovačko lake (leg. M. Grbović 01-jul1979, 2208, n.spec. = 3); (leg. M. Radovanović 11-aug-1961, 1477, n.spec. = 6); Mt. Njegoš: Donje Crkvice (v.), Valovi (leg. G. Džukić, M. Kalezić 09-jun-2008, 2177, n.spec. = 3); Gornja Trepča (v.), Donja Trepča, wellheads (leg. G. Džukić, M. Kalezić 08-jun-2008, 2174, n.spec. =6); Novice Kovača puddle (leg. G. Džukić, M. Kalezić 08-jun-2008, 2179, n.spec. =2); Roganovića Voda (leg. G. Džukić, M. Kalezić 08-jun-2008, 2170, n.spec. =2); Gornje Crkvice (v.), Turuntaš (leg. G. Džukić, M. Kalezić 11jun-2005, 2479, n.spec. = 6); Mt. Prekornica: Ćetni Do (leg. G. Džukić, M. Kalezić 23-aug-2001, 2325, n.spec. =9); Ponikvica (leg. G. Džukić, M. Kalezić 10-jun-1997, 2136, n.spec. =93); Suve Ponikvice (leg. G. Džukić, M. Kalezić 23-aug-2001, 2319, n.spec. = 31); Radole, Katranara (leg. G. Džukić, M. Kalezić 09-jun-1997, 1810, n.spec. =11); Mt. Sinjavina: Mali Starac (leg. G. Džukić, M. Kalezić 16jun-1983, 1503, n.spec. = 1); Zaboj (v.), Zabojsko lake (leg. G. Džukić, M. Kalezić 31-may-1984, 2663, n.spec. = 66); (leg. M. Radovanović 18-aug-1955, 1479, n.spec. = 3); Zminičko lake (leg. anonim. 18jun-1967, 2653, n.spec. =11); 07-jul-1979, 2485); s.d., 2652); (leg. G. Džukić 10-jul-1971, 1034, n.spec. = 14); (leg. M. Radovanović 04-aug-1959, 2527, n.spec. = 37); 23-aug-1960, 1309); Mt. Somina: Hercegova Luka (leg. G. Džukić, M. Kalezić 09-jun-2008, 2181, n.spec. = 36); Mt. Visitor: Bješkeća, lake (leg. P. Jakšić 03-aug-2004, 2192, n.spec. = 25); Mt. Žijovo: Bukumirsko lake (leg. A. Đorović 01-may-1989, 2654, n.spec. = 4); (leg. A. Ivanović 17-sep-1990, 1817, n.spec. = 64); 01-may1989, 2682); 01-may-1990, 2632); (leg. anonim. 11-jun-1949, 1848, n.spec. =19); 01-jun-1984, 1813); (2238); 04-jun-1995, 1808); (1814); 01-aug-1949, 1279); 28-may-2003, 2300); s.d., 224); (leg. D. Kiković 25-sep-1976, 1466, n.spec. = 16); (leg. G. Džukić 21-aug-1977, 1629, n.spec. = 3); (leg. G. Džukić, M. Kalezić 01-jun-1984, 2422, n.spec. = 272); 03-jun-2005, 2476); 22-aug-2002, 2494); 28-may-2003, 2316); (leg. M. Radovanović 28-aug-1949, 1291, n.spec. = 20); (1477); Katun Lokva challet (leg. G. Džukić, M. Kalezić 01-jun-1984, 2245, n.spec. = 58); lake near Bukumirsko lake (leg. G. Džukić 20-sep1991, 2659, n.spec. = 39); Ljuban (leg. B. Kokojevica, V. Mirčić 22-aug-1977, 1466, n.spec. = 51); Mutno 
lake (leg. G. Džukić, M. Kalezić 21-aug-2001, 2328, n.spec. =6); Kučka Korita (leg. M. Ćirović 24-jun1997, 1809, n.spec. = 6); Ljuđa (leg. anonim. 24-jun-1997, 2617, n.spec. =1); (leg. G. Džukić, M. Kalezić, R. Ćirović 21-aug-2001, 2484, n.spec. = 10); Rikavačko lake (leg. M. Busarčević 01-aug-2003, 2534, n.spec. = 1); Širokar (leg. G. Džukić, M. Kalezić 10-jun-1991, 2660, n.spec. = 66); (leg. G. Džukić, $M$. Kalezić, A. Ivanović 22-sep-1991, 2692, n.spec. = 70); Mts Prokletije: Bukumirsko - Rikavačko lake (leg. M. Radovanović 11-aug-1955, 1479, n.spec. = 1); Mt. Bogićevica, Ridsko lake (leg. anonim. 20-jul-1970, 1466, n.spec. = 23); (leg. G. Pasuljević 20-aug-1980, 1466, n.spec. = 8); (leg. M. Radovanović 29-aug-1954, 1477, n.spec. = 5); Mt. Mokra Gora (leg. G. Džukić 10-jun-1977, 353, n.spec. =13); Mt. Visitor (leg. anonim. 21-sep-1967, 1441, n.spec. = 9); Širokar (leg. G. Džukić, M. Kalezić 18-sep-1990, 2661, n.spec. = 323); Vusanje (v.), Podkobila lake (leg. M. Niketić 14-jul-1995, 1691, n.spec. =7); Savina Voda (leg. G. Džukić 09-jun-1972, 353, n.spec. = 4); Nikšić - Plužine: Bajovo Polje (v.) (leg. G. Džukić, M. Kalezić 07-jun-2008, 2232, n.spec. = 8); Pljevlja (leg. R. Malidžan, M. Radoš 25-nov-1976, 1466, n.spec. = 2); Gradac (leg. R. Malidžan 10-aug-1976, 1466, n.spec. = 2); Podgorica: Mt. Brotnjik, Ubina, Mrcki Uba (leg. M. Kalezić, G. Džukić, M. Marković 21-jun-2004, 2227 , n.spec. =7); Seoca (v.), Seocki Ublovi, Krnjačka Glavica (leg. M. Kalezić, M. Marković 21-jun2004, 2478, n.spec. $=14$ ); Prokletije: Vusanje (v.), Ćafa Bor pass, Čelića challet, Rustemova Livada (leg. anonim. 07-jul-1995, 1889, n.spec. = 6); Skadarsko lake: Krajina, Murići (v.) (leg. G. Džukić, M. Kalezić 15-jun-1997, 1805, n.spec. = 1); Šavnik: Pošćensko lake (leg. M. Radovanović 25jul-1947, 1479, n.spec. = 1); Toljenovica (leg. M. Radovanović 11-feb-1949, 1477, n.spec. =2); Vilusi: Banjani, Koprivice (v.), Crni Kuk (v.), Smrdelj puddle (leg. G. Džukić, M. Kalezić 11-jun-2005, 2334, n.spec. =12); Pilatovci (v.), Dolovi (v.), Nova Voda (leg. G. Džukić, M. Kalezić 10-jun-2005, 2483, n.spec. $=2$ ); Romania Bucharest: Gruiu (leg. anonim., s.d., 2810, n.spec. =1); Serbia (leg. G. Džukić, 19-sep-1969, 353, n.spec. =9); (leg. G. Pasuljević s.d., 1466, n.spec. =4); Bosilegrad: Božica (v.) (leg. M. Kalezić, G. Džukić, M. Marković 19-sep-2006, 2226, n.spec. =20); Brankovci (v.), Zli Dol (v.), Ezerine pond (leg. M. Kalezić 24-apr-2014, 2383, n.spec. = 29); Zli Dol (v.) (leg. G. Džukić, M. Kalezić 26-sep-2005, 2311, n.spec. =17); Crna Trava: Biljine Bare (leg. anonim. 21-sep-2007, 2195, n.spec. = 4); (leg. G. Džukić, M. Kalezić 23-jun-2007, 2169, n.spec. = 31); Gnjilane (leg. D. Jović 10-aug1981, 357, n.spec. = 5); Kosovo (leg. G. Pasuljević s.d., 357, n.spec. =1); Loznica: Zajača (v.) Paskovac (v.) (leg. M. Popović-Fant 12-mar-1990, 2694, n.spec. = 116); Ljubovija: Gledeće (v.) (leg. J. Crnobrnja-Isailović 16-apr-1995, 2138, n.spec. = 8); 05-apr-1998, 1680); Majdanpek: Debeli Lug (v.), Todorova river (leg. G. Džukić, M. Kalezić 27-apr-2009, 2144, n.spec. =2); (leg. S. Marinković 15-mar-1990, 1037, n.spec. = 1); Mt. Čemernik: Bele Rovine (leg. G. Džukić, M. Kalezić 24-jun-2007, 2172, n.spec. = 13); Mlačište (v.) (leg. G. Džukić, M. Kalezić 15-jun-2006, 2219, n.spec. =9); Kesino lake (leg. G. Džukić, M. Kalezić 15-jun-2006, 2541, n.spec. =1); Mlačǐške Meane (leg. G. Džukić, M. Kalezić 15-jun-2006, 2225, n.spec. = 4); Pavličane (v.), Bela Voda (leg. G. Džukić, M. Kalezić 20-apr2007, 2167, n.spec. = 4); Mt. Golija: Dajićko lake (leg. B. Curčić 30-apr-2001, 1191, n.spec. =9); Okruglica (v.), lake (leg. G. Džukić, M. Kalezić 14-jun-2001, 1193, n.spec. =29); Mt. Javor: Bukovik (v.) (leg. G. Džukić, M. Kalezić 27-aug-2001, 1194, n.spec. =15); Šančevi puddle (leg. M. Paunović 26may-1994, 1689, n.spec. = 2); Vasilin Vrh (leg. M. Paunović 16-may-1994, 2132, n.spec. =7); Mt. Južni Kučaj: Ciganska Bara, Ljubina Bara (leg. G. Džukić, M. Kalezić 16-may-2000, 1681, n.spec. = 30); Mt. Kopaonik (leg. M. Radovanović 07-jul-1951, 1469, n.spec. = 1); Jablanova Ravan (leg. J. Crnobrnja-Isailović 18-may-1986, 353, n.spec. = 8); Mt. Kukavica: Jasikov Rid (leg. G. Džukić 18may-1977, 353, n.spec. = 10); Mt. Maljen: Divčibare, Pecina Bara (leg. J. Crnobrnja-Isailović 24-jul1999, 1103, n.spec. = 4); Mt. Ostrozub: Ruplje (v.) (leg. anonim. s.d., 1694, n.spec. = 2); (leg. G. Džukić, M. Kalezić 12-sep-2006, 2296, n.spec. = 1); 01-mar-2007, 2162); s.d., 1054); Bankovci (v.) (leg. G. Džukić, M. Kalezić 31-mar-2007, 2159, n.spec. = 2); Selišta (leg. G. Džukić, M. Kalezić 20-apr-2007, 2176, n.spec. = 28); Zeleniče (leg. G. Džukić, M. Kalezić 22-jun-2007, 2155, n.spec. =1); Mt. Tara: Beli Rzav lake (leg. G. Džukić 15-aug-1982, 353, n.spec. = 2); lake (leg. G. Džukić 21-oct-1977, 353, n.spec. = 1); Miloševac (leg. anonim. 16-may-1972, 353, n.spec. =1); Mitrovac (v.), Crveni Potok stream (leg. G. Džukić 15-aug-1982, 353, n.spec. = 7); (1682); Pastište (v.), Požar hamlet (leg. G. Džukić 21-may-1972, 353, n.spec. = 2); Zaovine (v.), Beli Rzav lake (leg. G. Džukić 20-may-1972, 353, n.spec. = 10); Mt. Zlatar (leg. G. Džukić 25-may-1978, 353, n.spec. =1); Akmačići (v.) (leg. J. Crnobrnja-Isailović 19-aug-1996, 1092, n.spec. = 2); Aljinovići (v.), Goveđak (leg. G. Džukić 11-sep1991, 1530, n.spec. $=28$ ); Okutnjica (leg. anonim. 07-jul-1996, 1024, n.spec. $=21$ ); (leg. J. CrnobrnjaIsailović 07-jul-1996, 2623, n.spec. = 1); Popovići (v.) (leg. J. Crnobrnja-Isailović 21-aug-1996, 2312, 
n.spec. = 7); Mts Prokletije (leg. V. Radičević s.d., 1261, n.spec. = 55); Kosovo side (leg. G. Pasuljević s.d., 1259, n.spec. = 75); Raški Do (leg. G. Džukić, B. Milošević 17-jul-1980, 1466, n.spec. = 2); Mts Šar-Planina (leg. anonim. s.d., 2696, n.spec. = 40); Mt. Konjuška, Mala Planina (leg. $D$. Komatina 12-jul-1981, 357, n.spec. =6); several lakes (leg. G. Džukić, M. Kalezić, J. Crnobrnja-Isailović 01-jul-1995, 559, n.spec. = 41); Šutman, Belo lake (leg. Pljakić 13-aug-1954, 1468, n.spec. = 13); Vraca, Donje Ravne Mlake (leg. G. Džukić, M. Kalezić 27-jul-1995, 1195, n.spec. = 19); (leg. J. Crnobrnja-Isailović, M. Kalezić, G. Džukić 28-jul-1995, 1891, n.spec. =1); Nova Varoš (leg. G. Džukić, M. Kalezić 27-aug-2001, 2310, n.spec. = 13); Paraćin: Glavice (v.) (leg. G. Džukić, M. Kalezić 11-jun2005, 2482, n.spec. = 6); Pešter Plateau: Delimeđe (v.) (leg. G. Džukić, S. Cvetkov 01-may-1979, 1228 , n.spec. $=1)$; (leg. S. Cvetkov 19-may-1979, 1032, n.spec. =10); Mt. Jarut (leg. anonim. 29-apr2010, 2207, n.spec. =6); Prijepolje: Gornje Babine (v.) (leg. M. Grbović s.d., 2644, n.spec. = 26); Priština: Gračaničko lake (leg. D. Kiković 18-sep-1976, 1466, n.spec. = 2); Obilić (leg. G. Pasuljević 01-apr-1967, 1262, n.spec. = 3); (1475); Stari Vlah: Sjenica, Duga Poljana (v.) (leg. J. CrnobrnjaIsailović 30-aug-1997, 2669, n.spec. = 7); Surdulica: Kostroševci (v.) (leg. anonim. 01-apr-2007, 2148, n.spec. = 1); Užice: Tatinac (v.) (leg. Ž. Tomanović 01-apr-1990, 1527, n.spec. = 8); Užička Požega: Užička Ježevica (v.), Mađer (v.) (leg. M. Marković 01-may-1986, 2646, n.spec. = 6); Valjevo: Gola Glava (v.) (leg. G. Džukić, M. Kalezić 26-apr-1998, 1676, n.spec. = 2); Joševa (v.) (leg. G. Džukić, M. Kalezić 26-apr-1998, 1019, n.spec. = 40); (1683); 14-apr-2005, 1684); Kamenica (v.) (leg. N. Kalezić 16-may-1992, 1679, n.spec. = 7); Koceljeva (leg. N. Pantelić 26-apr-1992, 1614, n.spec. = 2); Rudna Glava (v.) (leg. G. Džukić, M. Kalezić 24-apr-1998, 2134, n.spec. =10); Vlasina (leg. G. Džukić, M. Kalezić 23-apr-2005, 1688, n.spec. =8); 13-sep-2006, 2298); 01-apr-2007, 2166); 20may-1981, 2658); canal (leg. G. Džukić, M. Kalezić 21-jun-2007, 2214, n.spec. = 16); gorge (leg. G. Džukić, M. Kalezić 21-sep-2007, 2202, n.spec. = 7); Klisura (v.) (leg. anonim. s.d., 1807, n.spec. = 2); (leg. G. Džukić, M. Kalezić 21-sep-2007, 1255, n.spec. = 10); Ljutež (v.) (leg. G. Džukić, M. Kalezić 19apr-2007, 2158, n.spec. = 3); Pavličane (v.), Ševarka (leg. G. Džukić, M. Kalezić 20-apr-2007, 1084, n.spec. = 1); Stojkovića Mahala (v.), Đumurkana (leg. G. Džukić, M. Kalezić 20-may-1981, 1197, n.spec. = 26); Tumba, Tumba I (leg. G. Džukić, M. Kalezić 22-jun-2007, 1059, n.spec. = 3); Vlasinsko lake (leg. anonim. 16-oct-1991, 1315, n.spec. = 1); Vranje: Drenovac (v.), Markovo Kale (leg. G. Džukić, M. Kalezić 14-may-1980, 1196, n.spec. = 79); Markovo Kale (leg. anonim. 14-may-1980, 2133, n.spec. = 47); Mt. Krstilovica, Devotin, lake (leg. M. Denoel, G. Džukić, M. Kalezić 24-sep-2005, 2543, n.spec. = 1); Slovenia Julian Alps: Mt. Triglav, Ovčarija (leg. anonim., 26-sep-1989, 692, n.spec. = 15); Triglavsko lake (leg. M. Radovanović 26-aug-1955, 2474, n.spec. =1); Triglavsko lake, Crno lake (leg. M. Radovanović 27-aug-1955, 1479, n.spec. = 1); Ljubljana: Ig (v.) (leg. G. Džukić 01-apr1979, 1820, n.spec. = 34); Kremenica (leg. anonim. 28-apr-1981, 2497, n.spec. = 50); (leg. G. Džukić, M. Kalezić 28-apr-1981, 2650, n.spec. = 40); Mt. Pohorje: Lovrenška lakes (leg. B. Kryštufek 08-jun1905, 726, n.spec. = 3); Rogaška Slatina: Gostišče Bellevue (leg. G. Džukić, M. Kalezić 26-may1987, 2251, n.spec. $=10)$.

\section{Lissotriton helveticus}

France Larzac (leg. M. Denoel, 2008, 2787, n.spec. = 6); Azirou-Croix (leg. M. Denoel 2008, 2807, n.spec. $=2$ ); Mas De Jourdes (leg. M. Denoel 2008, 2809, n.spec. = 1); Salvetat (leg. $M$. Denoel 2007, 2343, n.spec. = 3); Trailles (leg. M. Denoel 2006, 2808, n.spec. =1).

\section{Lissotriton montandoni}

Poland (leg. J. Szymura, 04-jun-1982, 2244, n.spec. $=8$ ); Romania Bacau: Mt. Nemira, Valea Groazei (leg. D. Cogalniceanu, 27-may-1989, 2357, n.spec. $=11$ ).

\section{Lissotriton vulgaris}

S.I. (leg. anonim., s.d., 293, n.spec. = 1); (539); 1005); (1311); 1312); (1364); 1365); (1480); 1864); (2175); 2228); (2242); 2248); (2421); 2563); (2595); 2608); (2609); 2639); (leg. G. Džukić s.d., 352, n.spec. =3); (1271); (1272); (1499); 2224); (2344); 2377); (leg. M. Radovanović 01-aug-1959, 1471, 
n.spec. = 17); Gornje (leg. anonim. s.d., 2592, n.spec. = 31); Tabovci (leg. anonim. 17-may-1989, 1486, n.spec. = 2); Bosnia and Herzegovina Bosansko Grahovo: Korana stream (leg. G. Džukić, M. Kalezić, E. Kletečki, 10-apr-1989, 2568, n.spec. = 47); Domanovići (leg. anonim. 01-apr-1981, 2517 , n.spec. = 40); Glamoč (leg. G. Džukić, M. Kalezić 18-may-1983, 2625, n.spec. = 14); Kupres (leg. G. Džukić, M. Kalezić 17-may-1983, 2566, n.spec. =60); (2748); Bukavička Previja, lake (leg. G. Džukić 05-jun-1985, 2522, n.spec. = 53); Kurića Poljane (leg. G. Džukić 04-jun-1985, 2640, n.spec. = 1); Mt. Stožer, Mračajsko lake (leg. G. Džukić 05-jun-1985, 594, n.spec. =1); (2772); Mt. Igman (leg. G. Džukić 01-aug-1974, 1281, n.spec. = 7); Mt. Zelengora: Tjentište (v.) (leg. G. Džukić 13may-1983, 1498, n.spec. = 12); Nevesinje: Dabarovo Polje, Berkovići (leg. anonim. 14-may-1983, 2206, n.spec. = 5); Nevesinjsko Polje, Seljani (v.) (leg. anonim. 20-apr-1991, 2204, n.spec. =66); Sopilja (v.), chasm (leg. anonim. 16-may-1983, 2502, n.spec. $=70$ ); Sarajevo: Kijevo (v.), Železnica river (leg. G. Džukić 12-may-1983, 694/1, n.spec. = 5); Teslić: Jelah (v.) (leg. M. Kalezić 01-apr-1980, 2572, n.spec. = 57); Trebinje: Turice (v.), Lokva (leg. M. Plećaš 03-jun-2005, 1835, n.spec. =1); Croatia Baranja: Beli Manastir (leg. M. Kalezić, 26-apr-1980, 2266, n.spec. =76); Drniš (leg. anonim. 01-may-1987, 2511, n.spec. = 75); Gospić: Lički Osik (v.) (leg. G. Džukić 01-jun1985, 2586, n.spec. = 68); (leg. G. Džukić, M. Kalezić 12-apr-1989, 2605, n.spec. = 19); (leg. M. Kalezić 01may-1980, 2565, n.spec. = 33); Smiljan (v.) (leg. G. Džukić 02-jun-1985, 2272, n.spec. = 47); llok (leg. G. Džukić 15-apr-1981, 2265, n.spec. = 28); Istra: Motovun, Istarske Toplice spa (leg. anonim. 31-may-1991, 2510, n.spec. = 72); Salakovci (v.) (leg. G. Džukić, M. Kalezić 27-apr-1981, 2274, n.spec. = 24); (2564); Svetvinčenat (v.), Velika Vala (leg. G. Džukić, M. Kalezić 01-apr-1991, 2585, n.spec. = 97); Karlovac (leg. N. Tvrtković 12-apr-1989, 1427, n.spec. = 1); (2569); Koprivnica: Peteranec (v.) (leg. anonim. 05-apr-1988, 715, n.spec. = 13); Krbavsko Polje (leg. G. Džukić, M. Kalezić 13-apr1989, 2573, n.spec. $=25$ ); Landonov Gaj (leg. anonim. 13-apr-1989, 2505, n.spec. = 67); Krk (ins.) (leg. anonim. 01-apr-1981, 2616, n.spec. = 31); Bag (leg. A. Đorović, G. Džukić, M. Kalezić 01-apr-1991, 2275 , n.spec. = 82); Diviska (v.) (leg. anonim. 01-apr-1990, 2618, n.spec. = 54); 05-apr-1991, 2506); (2520); Juran Dvor (leg. A. Đorović 01-aug-1989, 2264, n.spec. =73); (2376); (leg. A. Ivanović 01-aug1989, 310, n.spec. =1); Ljubas (leg. M. Kalezić, A. Ivanović, D. Bejaković 04-apr-1991, 2614, n.spec. = 43); Ponikve lake (leg. M. Radovanović 24-jul-1965, 2474, n.spec. =1); Punat (v.), above (leg. G. Džukić 26-apr-1981, 352, n.spec. =4); Metković (leg. G. Džukić, M. Kalezić 07-apr-1989, 2606, n.spec. = 51); Mt. Papuk: Jankovac (leg. G. Džukić, M. Kalezić 01-may-1981, 2269, n.spec. = 40); Otočac: Švica (v.) (leg. G. Džukić, M. Kalezić s.d., 2273, n.spec. = 10); (leg. M. Kalezić 01-apr-1979, 2583, n.spec. = 30); Vrhovine (v.), Borića Borik (leg. anonim. 14-sep-1961, 1467, n.spec. = 3); Ravni Kotari: Brgud (v.) (leg. G. Džukić, M. Kalezić 10-apr-1989, 2193, n.spec. = 35); Smilčići (v.) (leg. anonim. 02jun-1985, 962, n.spec. =1); 11-apr-1989, 2508); (leg. G. Džukić, M. Kalezić 24-may-1987, 345, n.spec. = 17); Rijeka: Kukuljanovo (v.) (leg. G. Džukić 25 -apr-1981, 1434, n.spec. = 2); Lipa (v.) (leg. G. Džukić 25-apr-1981, 1303, n.spec. = 2); (1427); 2276); (leg. G. Džukić, M. Kalezić 25-apr-1981, 2597, n.spec. =27); Mamići (v.), Macanova Lokva puddle (leg. G. Džukić, M. Kalezić 08-apr-1989, 2670, n.spec. = 27); Plosna (v.) (leg. G. Džukić, M. Kalezić 25-apr-1981, 2620, n.spec. =27); (2628); Sinj: Sinjsko Polje (leg. G. Džukić, M. Kalezić 09-apr-1989, 2375, n.spec. =67); (leg. G. Džukić, M. Kalezić, N. Tvrtković 09-apr-1989, 659, n.spec. = 78); Otok (leg. G. Džukić 22-may-1987, 1493, n.spec. = 2); Sisak: Mokro Polje (v.), Mlječko Polje (leg. I. Ham 27-apr-1990, 2388, n.spec. = 7); Velika Gorica: Peščenica (v.) (leg. G. Džukić 24-apr-1981, 1611, n.spec. = 5); (2778); Turopolje, Orle (v.) (leg. G. Džukić 23-apr-1981, 1303, n.spec. = 4); (leg. G. Džukić, M. Kalezić 01-apr-1981, 2588, n.spec. = 39); 23apr-1981, 2247); (leg. G. Džukić, N. Tvrtković 18-apr-1986, 2613, n.spec. = 29); Macedonia Bitolj: Streševo (leg. anonim., s.d., 2201, n.spec. = 3); (leg. G. Džukić, M. Kalezić 15-may-1980, 2254, n.spec. = 65); (2598); 16-may-1980, 2581); Dojran (leg. M. Radovanović 21-may-1958, 2474, n.spec. = 8); Suva Reka accumulation (leg. G. Džukić 08-may-1997, 2498, n.spec. = 24); (leg. G. Džukić, M. Kalezić 08may-1997, 2369, n.spec. = 10); Kumanovo: Brzak (v.), Izvorište Brzak (leg. G. Džukić 04-mar1999, 1695, n.spec. = 5); Kumanovo - Kriva Palanka: Stracin (v.), Karadag, Suvo lake (leg. G. Džukić 25-may-2004, 1826, n.spec. =1); Suvo lake (leg. G. Džukić, M. Kalezić 10-may-1997, 2525, n.spec. = 53); Mt. Galičica (leg. G. Džukić 08-may-1997, 2374, n.spec. = 14); Kostobačilo, Mečkina Lokva puddle (leg. anonim. 14-jun-1981, 2454, n.spec. = 1); Lokva (leg. Bio Eko 21-may-2002, 2379, n.spec. = 7); Skoplje (leg. anonim. s.d., 2197, n.spec. = 7); Rašče (v.) (leg. M. Radovanović 10-may1959, 2474, n.spec. = 2); Struga: Struško Pole, Misleševo (v.) (leg. anonim. 07-may-1997, 1451, 
n.spec. = 2); Strumica: Strumičko Pole (leg. M. Kalezić, B. Kryštufek 20-apr-1990, 2509, n.spec. = 33); Montenegro (leg. anonim., 16-oct-1996, 2767, n.spec. =1); s.d., 1318); Berane [“Ivangrad"] (leg. S. Ristić 23-may-1983, 351, n.spec. = 15); (1466); Budva: Grbalj, Sušica river, Mrčevo Polje (leg. B. Mićković 29-jan-1995, 1892, n.spec. =2); Cetinje: Bjeloši (v.), Lašor puddle (leg. G. Džukić, M. Kalezić 30-may-1990, 720, n.spec. = 25); Ćeklići, Kućišta (v.), Crno Korito (leg. M. Kalezić, G. Džukić, M. Marković 20-jun-2004, 2440, n.spec. =6); Kućišta (v.), Ublica (leg. M. Kalezić, G. Džukić, M. Marković 20-jun-2004, 2523, n.spec. = 23); Resna (v.) (leg. anonim. 10-apr-1997, 2617, n.spec. = 1); s.d., 1693); (leg. G. Džukić, M. Kalezić 14-oct-1996, 1867, n.spec. =1); s.d., 2016); Rid (leg. G. Džukić, M. Kalezić 13-mar, 1087, n.spec. = 15); Dobrsko (v.) (leg. G. Džukić, M. Kalezić 04-apr-1989, 2556, n.spec. = 2); Cetinje - Grahovo: Male Cuce, Trnjine (v.) (leg. M. Marković, G. Džukić, M. Kalezić 29-may-2003, 2339, n.spec. = 10); Ržani Do (v.), Cape (leg. M. Kalezić, G. Džukić, M. Marković 20-jun-2004, 952, n.spec. =4); Cucka Bata (leg. anonim. 31-may-1990, 1325, n.spec. =6); Danilovgrad: Blizanci (leg. G. Džukić, M. Kalezić 25-apr-2002, 2368, n.spec. =1); Čevo (v.), Čevske Ubli (v.) (leg. M. Kalezić, G. Džukić, M. Marković 19-jun-2004, 2524, n.spec. = 19); Lazine (leg. G. Džukić, A. Ivanović, M. Kalezić 15mar-2008, 2513, n.spec. = 30); Kosić (leg. G. Džukić, M. Kalezić 26-apr-2002, 2340, n.spec. = 3); Milojevići (v.), Grabovica Ubao (leg. anonim. 06-jun-2005, 2514, n.spec. =4); Mrainjac (leg. G. Džukić, M. Kalezić 06-jun-2005, 2322, n.spec. = 5); Mt. Garač (leg. M. Kalezić, G. Džukić, M. Marković 29may-2003, 2289, n.spec. = 45); Donja Markovina (v.) (leg. G. Džukić, M. Kalezić 25-apr-2002, 2364, n.spec. = 3); Erakovice (leg. M. Kalezić, G. Džukić, M. Marković, M. Ćirović, D. Šipčić 29-may-2003, 2281, n.spec. = 13); Vodine (leg. G. Džukić, M. Kalezić 25-apr-2002, 2137, n.spec. = 26); Rujišta, Bezimena (leg. G. Džukić, M. Kalezić 25-apr-2002, 2370, n.spec. =1); Zagarač (v.), Cicmanovica (leg. G. Džukić, M. Kalezić 25-apr-2002, 2366, n.spec. = 8); Čelo (leg. G. Džukić, A. Ivanović, M. Kalezić 25-apr-2002, 2365, n.spec. = 8); Vodine (leg. G. Džukić, I. Aleksić, J. Crnobrnja-Isailović, M. Kalezić 24-may-1996, 2589, n.spec. = 13); Grahovo: Donji Izvor (v.) (leg. M. Kalezić, G. Džukić, M. Marković 20-jun-2004, 2512, n.spec. = 5); Gornji Izvor (v.) (leg. M. Kalezić, G. Džukić, M. Marković 20-jun-2004, 2282, n.spec. = 5); Građevina Vučetića (v.) (leg. G. Džukić, M. Kalezić 15-jun-1983, 2220, n.spec. = 42); Kotlići (v.) (leg. G. Džukić, M. Kalezić 15-jun-1983, 1453, n.spec. = 11); Razmuđe (v.), Razmućski Dol, Razmućska Kamenica (leg. G. Džukić, M. Kalezić 20-jun-2004, 2301, n.spec. = 2); (leg. M. Kalezić, G. Džukić, M. Marković 20-jun-2004, 1293, n.spec. = 10); Rokoči (v.), Meka Lokva (leg. M. Kalezić, G. Džukić, M. Marković 20-jun-2004, 2291, n.spec. = 11); Grahovo - Vilusi: Velika Osječenica (v.) (leg. anonim. 07-jun-1984, 2237, n.spec. =1); 01-may-1980, 2161); 23-aug-1982, 2216); (leg. G. Džukić 10-jun-1980, 1429, n.spec. = 1); 12-apr-1981, 2157); 15-sep-1986, 2205); 08-aug-1970, 2594); 29-aug2002, 2332); 31-may-1990, 1851); (leg. G. Džukić, M. Kalezić 12-apr-1981, 1347, n.spec. = 33); 01-apr1983, 1003); (2600); (leg. M. Kalezić, G. Džukić, M. Marković 31-may-1990, 2210, n.spec. = 11); Osječenica puddle (leg. G. Džukić, M. Kalezić 06-sep-1991, 2243, n.spec. = 189); 22-oct-1983, 2255); 23-aug-2001, 2371); (leg. V. Kalafatić 17-jun-1984, 2257, n.spec. = 49); Herceg Novi: Sutorina (v.) (leg. A. Hegediš 10-aug-1990, 1490, n.spec. = 2); Kolašin: Uljara (leg. anonim. 06-jul-1979, 2698, n.spec. =24); Krivošije: Ubli (v.), Sunjava (leg. G. Džukić, M. Kalezić 28-apr-2002, 2363, n.spec. =6); Mt. Bioč: Stabna (v.), Stubica (v.), Brštevac (leg. G. Džukić, M. Kalezić 06-jun-2008, 2231, n.spec. =1); Mt. Bjelasica: Biogradska Gora, Jezerišta (leg. M. Stanković 25-sep-1994, 217, n.spec. = 1); Lalev Potok stream (leg. M. Stanković 25-sep-1994, 217, n.spec. = 2); Biogradsko lake (leg. G. Džukić, J. Crnobrnja-Isailović 07-jul-1996, 2360, n.spec. = 1); 08-jul-1996, 1678); Šiško lake (leg. M. Radovanović 12-aug-1948, 2474, n.spec. = 1); Mt. Durmitor (leg. anonim. s.d., 682, n.spec. = 17); (1314); 1400); Valovito lake (leg. A. Ćetković 29-may-2003, 2535, n.spec. =1); Zminje lake (leg. M. Kalezić 11-jul-1977, 2263, n.spec. = 42); Žabljak, Barno lake (leg. Momir Paunović 18-jul-2006, 1836, n.spec. = 1); Crno lake - Zminje lake (leg. V. Anđelković 01-jul-1958, 1861, n.spec. = 8); Mt. Lovćen (leg. anonim. 14-aug-1984, 2458, n.spec. = 3); lake (leg. anonim. 28-may-1990, 1284, n.spec. =24); Mt. Lovćen, lake under (leg. anonim. 02-jun-1984, 2731, n.spec. =1); Mt. Lovćen lake (leg. anonim. 02-jun-1984, 2601, n.spec. = 65); Mt. Njegoš: Gornja Trepča (v.), Donja Trepča, wellheads (leg. G. Džukić, M. Kalezić 08-jun-2008, 2174, n.spec. = 5); Novice Kovača puddle (leg. G. Džukić, $M$. Kalezić 08-jun-2008, 2179, n.spec. = 4); Roganovića Voda (leg. G. Džukić, M. Kalezić 08-jun-2008, 2170, n.spec. =2); Mt. Orjen: Balješina Lokva puddle (leg. M. Kalezić 15-oct-1977, 2621, n.spec. = 75); Kruševice (v.), Lokanj (leg. anonim. 01-may-1980, 2211, n.spec. = 3); s.d., 2213); (leg. M. Kalezić 02-may-1980, 2252, n.spec. = 47); (2575); Mt. Rumija: Sutorman (v.), Lera (leg. G. Džukić, M. 
Kalezić 02-jun-1990, 1523, n.spec. =26); Mt. Sinjavina: Zaboj (v.) (leg. anonim. 31-may-1984, 1489, n.spec. = 5); Zminičko lake (leg. anonim. 07-jul-1979, 2485, n.spec. = 1); (leg. G. Džukić 21-jul-1975, 1630, n.spec. =1); (leg. G. Džukić, M. Kalezić 01-jul-1977, 2624, n.spec. = 57); (leg. M. Radovanović 04aug-0159, 1479, n.spec. = 2); Mt. Žijovo: Bukumirsko lake (leg. anonim. 25-sep-1976, 351, n.spec. = 1); 28-may-2003, 2300); (leg. G. Džukić 20-sep-1991, 2659, n.spec. = 17); (leg. G. Džukić, M. Kalezić 28may-2003, 2316, n.spec. $=6$ ); s.d., 2750); challet (leg. anonim. 01-jun-1984, 2503, n.spec. $=47$ ); Mts Prokletije: Ropojana, Lićeni Gštars lake (leg. anonim. 02-jul-1984, 2725, n.spec. =1); (leg. G. Džukić 02-jul-1984, 1050, n.spec. =2); Nikšić: Bijele Poljane (leg. G. Džukić, M. Marković, M. Kalezić 29-may-2003, 2362, n.spec. =2); Nikšić - Plužine: Bajovo Polje (v.) (leg. G. Džukić, M. Kalezić 07jun-2008, 2232, n.spec. = 8); Podgorica: Komani, Ćafa, Smrdan (leg. G. Džukić, M. Kalezić 26-apr2002, 2367, n.spec. =1); Vojova Kamenica (leg. G. Džukić, M. Kalezić 26-apr-2002, 2372, n.spec. = 4); Progonovići (v.), Gornji Stabnovi (leg. G. Džukić, M. Kalezić 22-aug-2001, 2361, n.spec. = 2); Releza (v.), Razvađa puddle (leg. G. Džukić, M. Kalezić 24-apr-2002, 2378, n.spec. = 5); Rijeka Crnojevića: Ceklin (v.), Brežina (v.), Donji Lokanj (leg. G. Džukić, M. Kalezić 17-sep-1990, 2629, n.spec. = 194); Gornji Ceklin (v.) (leg. G. Džukić, M. Kalezić 19-apr-1991, 2260, n.spec. =74); Jankovića Krš (v.), Pištet (leg. anonim. 14-jul-1992, 1491, n.spec. = 24); Skadarsko lake: Krajina, Gornja Briska (v.) (leg. G. Džukić, M. Kalezić 21-apr-1991, 1430, n.spec. = 1); Koštanica (v.) (leg. anonim. 25-apr-1991, 1478, n.spec. = 4); (leg. G. Džukić, M. Kalezić 21-apr-1991, 2582, n.spec. = 42); Livari (v.) (leg. I. Aleksić, J. Crnobrnja-Isailović 30-may-1996, 2580, n.spec. =12); Šavnik: Pošćensko lake (leg. G. Džukić, V. Vasić 20-jul-1975, 334, n.spec. = 3); Tivat: Tivatske Solane, Lješevići (v.) (leg. A. Hegediš 08-apr-1990, 2571, n.spec. = 30); Ulcinj: Donji Štoj (v.) (leg. G. Džukić 02-apr-1989, 2684, n.spec. =1); (leg. G. Džukić, M. Kalezić 02-apr-1989, 2630, n.spec. =61); Vilusi: Banjani, Bojanje Brdo (v.) (leg. G. Džukić, M. Kalezić 04-jun-1984, 2604, n.spec. = 53); Građevina (v.) (leg. G. Džukić, M. Kalezić 12-apr-1981, 2627, n.spec. = 33); 04-jun-1984, 2261); Podljut (v.), Kadaruša (leg. G. Džukić, M. Kalezić 10-jun-2005, 2306, n.spec. = 4); Podljut (v.), Rudnjača (leg. G. Džukić, M. Kalezić 10jun-2005, 2358, n.spec. = 5); Spila, Voluje Oko (leg. G. Džukić, M. Kalezić 16-aug-1984, 2258, n.spec. = 5); Voluje Oko (leg. G. Džukić, M. Kalezić 05-jun-1984, 2603, n.spec. = 72); Ćetkova Kamenica (leg. G. Džukić, M. Kalezić 16-jun-1983, 1386, n.spec. =9); Kovačevića Lokva puddle (leg. G. Džukić, M. Kalezić 16-jun-1983, 2515, n.spec. =112); (2619); Pilatovci (v.) (leg. G. Džukić 26-jun-2004, 2320, n.spec. =1); Dolovi (v.), Nova Voda (leg. G. Džukić, M. Kalezić 10-jun-2005, 2483, n.spec. =9); Rutešića Voda (leg. G. Džukić, M. Kalezić 16-aug-1984, 2271, n.spec. =23); Virpazar: Orahovačko Polje (leg. G. Džukić, M. Kalezić 04-apr-1989, 2626, n.spec. = 70); Orahovštica stream (leg. G. Džukić 04-apr-1989, 2666, n.spec. = 3); Poland Mt. Kamionna (leg. J. Szymura, 01-may-1979, 2567, n.spec. = 22); Serbia (leg. anonim., s.d., 1801, n.spec. =4); Ada: Padej (v.) (leg. G. Mesaroš 01-may-1988, 697, n.spec. = 1); Babušnica: Gorčinci (v.) (leg. J. Crnobrnja-Isailović, I. Aleksić 02-jun-1998, 1649, n.spec. = 1); Resnik (v.) (leg. G. Džukić, M. Kalezić 11-apr-2008, 1207, n.spec. = 3); Bela Crkva: Češko Selo (v.), Fabijanski Potok stream (leg. M. Kalezić, G. Džukić, M. Marković 29-mar-2004, 1223 , n.spec. $=21$ ); Kaluđerovo (v.), Leskovački Potok stream (leg. G. Džukić, M. Kalezić 14-mar1999, 1227, n.spec. =1); (leg. M. Kalezić, G. Džukić, M. Marković 28-mar-2003, 1208, n.spec. =1); Siga canal (leg. M. Kalezić, G. Džukić, M. Marković 20-may-1998, 1662, n.spec. = 43); Kruščica (v.) (leg. G. Džukić, M. Kalezić 18-mar-2001, 1687, n.spec. = 12); (leg. M. Kalezić, G. Džukić, M. Marković 20-may-1998, 1672, n.spec. =6); Kusić (v.), Leskovačko lake (leg. M. Kalezić, G. Džukić, M. Marković 20-may-1998, 1642, n.spec. = 9); Lug stream (leg. M. Kalezić, G. Džukić, M. Marković 14-mar-1999, 1201, n.spec. =4); Mrtva Nera (leg. G. Džukić, M. Kalezić 05-apr-2003, 1212, n.spec. =2); Orešac (v.) (leg. M. Kalezić, G. Džukić, M. Marković 21-may-1998, 228, n.spec. = 3); Siga stream (leg. M. Marković s.d., 1018, n.spec. = 7); Stara Nera (leg. M. Marković 1-may-1987, 1232, n.spec. = 18); Beočin: Beočinske Livade (leg. G. Džukić 17-mar-1975, 1271, n.spec. = 2); Beograd: Dobanovci (v.) (leg. anonim. 28-apr-1995, 1509, n.spec. = 1); 01-sep-1996, 1697); 14-apr-1997, 2617); 29-apr-1997, 2617); 05-may-1995, 1507); 18-oct-1995, 1857); (leg. G. Džukić 1996, 247, n.spec. = 82); (leg. G. Džukić, M. Kalezić 26-apr-1993, 1444, n.spec. = 3); 17-jun-1995, 1020); 07-may-1995, 1015); 22-may-1993, 1445); (leg. I. Rot Nikčević 1996, 1673, n.spec. = 10); (leg. J. Crnobrnja-Isailović 27-mar-1995, 1500, n.spec. =1); 13-jun-1996, 1686); (leg. M. Ćirović 14-jul-1995, 1492, n.spec. = 10); Kanarevo Brdo (leg. N. Kokotović 17-may1981, 2574, n.spec. =2); Košutnjak (leg. M. Radovanović 05-apr-1949, 1471, n.spec. = 20); Krnjača (v.) (leg. S. Matvejev, G. Džukić 23-oct-1973, 1271, n.spec. = 1); Makiš (leg. anonim. 01-dec-1951, 1473, 
n.spec. = 5); Ostružnica (v.) (leg. anonim. 14-jun-1986, 1316, n.spec. = 1); Topčider (leg. V. Tomić s.d., 1467, n.spec. = 3); Voždovac, Kumodraški Potok stream (leg. S. Mičić 26-feb-1960, 1469, n.spec. =6); Blace (leg. anonim. s.d., 1481, n.spec. = 5); (leg. Veljković 07-mar-1978, 351, n.spec. = 4); Blacko lake (leg. G. Džukić 22-may-1977, 1271, n.spec. =2); Bosilegrad: Mlekominci (v.) (leg. J. Crnobrnja-Isailović, I. Aleksić 03-jun-1998, 1651, n.spec. =14); Zli Dol (v.) (leg. G. Džukić, M. Kalezić 16jun-2006, 1198, n.spec. = 6); Ezerine pond (leg. M. Kalezić 24-apr-2014, 2383, n.spec. = 4); Bujanovac: Moravica river (leg. G. Džukić 24-apr-2005, 2771, n.spec. =1); Čačak (leg. Domanović 01-mar-1965, 1471, n.spec. = 1); Ćuprija: Virine (v.) (leg. G. Džukić, M. Kalezić 12-may-2002, 1200, n.spec. = 5); Deliblato sands: Đurica (leg. M. Kalezić, G. Džukić, M. Marković 21-may-1998, 1663, n.spec. = 34); Stevanove Ravnice (leg. G. Džukić, M. Kalezić 27-feb-1999, 1677, n.spec. = 8); 18-jul2001, 2475); (leg. M. Kalezić 10-may-2003, 2317, n.spec. =1); Jasenovo (v.) (leg. G. Džukić, M. Kalezić 14-mar-1999, 1802, n.spec. = 2); Majur Bara (leg. G. Džukić 04-apr-1981, 1271, n.spec. = 1); Popina Bara (leg. G. Džukić, M. Kalezić 26-jun-1999, 1644, n.spec. =2); Utrine, Đabin Salaš (leg. G. Džukić, M. Kalezić 26-jun-1999, 1086, n.spec. = 1); Zamfir Bara (leg. G. Džukić, M. Kalezić 18-nov-2000, 1219, n.spec. =4); Despotovac: Vražja Bara (leg. G. Džukić, M. Kalezić 28-feb-1997, 2805, n.spec. = 2); (leg. G. Džukić, M. Kalezić, J. Radojičić 11-mar-1997, 1636, n.spec. = 36); Gnjilane: Kosovska Kamenica, Ograđe (leg. B.V. Milošević 28-apr-1980, 351, n.spec. = 2); Grdelička gorge: Migulovac (v.) (leg. G. Džukić, M. Kalezić 18-apr-2007, 1068, n.spec. =6); lake (leg. G. Džukić, M. Kalezić 18apr-2007, 2431, n.spec. = 2); Horgoš (leg. G. Džukić 08-apr-1982, 1423, n.spec. = 7); Bački Vinogradi (v.), Selevenjska Šuma forest (leg. G. Džukić, M. Kalezić 24-mar-1996, 1653, n.spec. = 1); Selevenjske Pustare (leg. G. Džukić, M. Kalezić 11-apr-1998, 1668, n.spec. = 3); Selevenjske Pustare, Kanas Bara (Lo Fej) (leg. G. Džukić, M. Kalezić 25-feb-1995, 328, n.spec. = 6); Jagodina (leg. anonim. 17-jul-1956, 1470, n.spec. = 39); Bunar (v.) (leg. G. Džukić, M. Kalezić 11-may-2002, 1199, n.spec. = 22); Ribnik (v.) (leg. G. Džukić, M. Kalezić 11-may-2002, 1205, n.spec. =1); Kikinda: Banatsko Veliko Selo (v.) (leg. G. Džukić 26-mar-2006, 2188, n.spec. =1); Kladovo: Manastirski Gaj (leg. anonim. 18-jun-1996, 975, n.spec. =6); Milutinovac (v.), Balta Lakuri (leg. G. Džukić, M. Kalezić 18-apr-2006, 1203, n.spec. =6); Zamna river (leg. G. Džukić, M. Kalezić 28-sep-2005, 2277, n.spec. =1); Kosovo (leg. G. Pasuljević s.d., 351, n.spec. =4); Kovin (leg. anonim. 09-may-1970, 1271, n.spec. = 1); Bavanište (v.) (leg. M. Živković 01-apr-1990, 1527, n.spec. = 6); Kragujevac (leg. $L$. Simić 1959, 1469, n.spec. = 7); Petrovac (v.) (leg. L. Simić 10-may-1259, 1469, n.spec. = 3); Leskovac: Vučje, Manetove Livade (leg. G. Džukić, M. Kalezić 16-jun-2001, 1105, n.spec. = 1); Loznica: Lešnica (v.) (leg. G. Džukić, M. Kalezić 23-mar-1991, 2615, n.spec. = 48); Paskovac (v.) (leg. M. Popović 05-apr-1990, 1038, n.spec. = 1); (leg. M. Popović-Fant 31-mar-1990, 1038, n.spec. = 1); Ljubovija: Duvanište (v.) (leg. J. Crnobrnja-Isailović, I. Aleksić 22-mar-1999, 1700, n.spec. = 2); Gledeće (v.) (leg. J. Crnobrnja-Isailović 16-apr-1995, 1645, n.spec. = 3); (leg. J. Crnobrnja-Isailović, I. Aleksić 05-apr-1998, 1666, n.spec. = 30); Majdanpek: Debeli Lug (v.), Todorova river (leg. G. Džukić, M. Kalezić 27-apr-2009, 2144, n.spec. = 1); Železnik (v.) - Debeli Lug (v.) (leg. V. Kalafatić 26-may-1980, 1633, n.spec. = 5); Mt. Avala: Trešnja (leg. anonim. s.d., 1042, n.spec. = 5); (leg. G. Džukić 04-jun-1979, 2671, n.spec. = 4); 05-apr-1983, 2587); (leg. G. Džukić, M. Kalezić 01-apr-1979, 1022, n.spec. = 128); lake (leg. anonim. 01-apr-1993, 1014, n.spec. = 16); Mt. Fruška Gora: Andrevlje (leg. anonim. 10-may-1996, 1505, n.spec. = 5); Mađarica (leg. G. Džukić 17-apr-1980, 2591, n.spec. = 23); Šuljam (v.) (leg. G. Džukić 16-apr-1980, 1028, n.spec. = 82); Mutalj hamlet (leg. G. Džukić 09-may-1980, 2500, n.spec. = 85); Testera (leg. G. Džukić 21-mar-1980, 1271, n.spec. =1); Vrdnik (v.), Vrdnička Kula (leg. G. Džukić 19-mar-1975, 1271, n.spec. = 2); Mt. Javor: Bukovik (v.) (leg. G. Džukić, M. Kalezić 27-aug-2001, 2380, n.spec. = 5); Šančevi puddle (leg. M. Paunović 26may-1994, 1689, n.spec. = 5); Mt. Kopaonik: lakes (leg. J. Crnobrnja-Isailović 01-may-1986, 1271, n.spec. = 3); Radošiće (v.) (leg. G. Džukić 28-apr-1984, 1071, n.spec. = 50); Semeteš (v.) (leg. J. Crnobrnja-Isailović 28-may-1986, 1271, n.spec. = 2); Mt. Maljen: Divčibare, Serđova Bara (leg. J. Crnobrnja-Isailović 26-jul-1999, 1225, n.spec. = 2); Mt. Miroč (leg. G. Džukić, M. Kalezić 17-may-2000, 1230, n.spec. = 1); Baluna (leg. G. Džukić, M. Kalezić s.d., 1843, n.spec. = 9); Brza Palanka (v.), Kilome (v.) (leg. G. Džukić, M. Kalezić 01-apr-2009, 1066, n.spec. =6); Cvetanovačka Bara (leg. G. Džukić, M. Kalezić 17-may-2000, 1202, n.spec. =6); Potrovo (v.), Majdan (leg. G. Džukić, M. Kalezić 25apr-2009, 1063, n.spec. = 14); Mt. Rtanj (leg. G. Džukić, M. Kalezić s.d., 2584, n.spec. = 68); Mt. Vršačke Planine (leg. G. Džukić, M. Kalezić 12-apr-2004, 1218, n.spec. =7); (2278); Česta (v.) (leg. 
anonim. 19-jul-1991, 2576, n.spec. =16); (leg. G. Džukić 31-mar-1983, 1011, n.spec. = 44); Česta (v.) and Mesić (v.) (leg. G. Džukić, M. Kalezić, A. Ivanović 03-aug-1991, 2610, n.spec. = 88); Mesić (v.) (leg. G. Džukić, M. Kalezić 18-may-1996, 1658, n.spec. = 3); Ribnjak (leg. G. Džukić, M. Kalezić 12-apr2004, 1215, n.spec. =1); Široko Bilo, Česta (leg. G. Džukić 14-jul-1991, 2184, n.spec. =1); Mt. Zlatar: Akmačići (v.) (leg. J. Crnobrnja-Isailović 19-aug-1996, 1092, n.spec. = 16); Aljinovići (v.), Goveđak (leg. G. Džukić 11-sep-1991, 1530, n.spec. =27); Okutnjica (leg. J. Crnobrnja-Isailović 07-jul1996, 2623, n.spec. = 41); Popovići (v.) (leg. J. Crnobrnja-Isailović 21-aug-1996, 2312, n.spec. = 7); Negotin (leg. anonim. 21-apr-1988, 2406, n.spec. = 7); 22-apr-1988, 1271); (2593); (leg. M. Kalezić, G. Džukić 16-may-2004, 690, n.spec. = 21); Sikole (v.) (leg. anonim. 21-jun-2003, 2471, n.spec. = 8); (leg. G. Džukić, M. Kalezić 16-may-2004, 1699, n.spec. = 2); (leg. J. Crnobrnja-Isailović, I. Aleksić 31-may-1998, 1660, n.spec. = 4); Sikolske Bare (leg. anonim. 18-jun-1980, 2707, n.spec. =1); Štubik (v.) (leg. G. Džukić, M. Kalezić 17-jun-1987, 1421, n.spec. = 4); 21-apr-1988, 2557); (leg. I. Aleksić, J. Crnobrnja-Isailović 31-may-1998, 1639, n.spec. = 1); (leg. J. Crnobrnja-Isailović, I. Aleksić 31-may-1998, 2669, n.spec. = 2); (leg. M. Kalezić, G. Džukić 21-apr-1988, 1271, n.spec. =1); Nova Varoš: Kokin Brod (v.), Neškoviće (v.), Šupljica (leg. G. Džukić 11-aug-1982, 2704, n.spec. = 13); Rutoši (v.) (leg. G. Džukić 13-aug-1982, 1271, n.spec. =14); Radoinjsko lake (leg. G. Džukić 10-aug-1982, 1271, n.spec. =1); Novi Bečej Melenci (v.): Taraška Đuglja (leg. anonim. 02-jul-1982, 1073, n.spec. =1); Novi Kneževac: Banatsko Aranđelovo (v.), Rabe (v.) (leg. G. Džukić, M. Kalezić 23-apr-1982, 1012, n.spec. =112); Obedska Bara (leg. G. Džukić, M. Kalezić 11-may-2004, 1698, n.spec. = 4); (leg. M. Kalezić 31-mar1980, 1010, n.spec. $=40$ ); Obrenovac: Starača, Kolubara river mouth (leg. G. Džukić, M. Kalezić 02jun-1997, 1685, n.spec. = 3); Umka (v.) (leg. M. Milenković 08-nov-2008, 1081, n.spec. =1); 02-dec2009, 2131); 01-oct-2007, 1804); Pećanska Bara (leg. M. Kalezić, G. Džukić 05-jul-1975, 1271, n.spec. = 4); Pančevo: Ivanovo (v.) (leg. G. Džukić, M. Kalezić 01-mar-1980, 1016, n.spec. = 25); Opovo (v.) (leg. A. Ivanović, M. Kalezić 15-apr-2005, 1690, n.spec. =6); Gergina Slatina (leg. G. Džukić, M. Kalezić 11-apr-2005, 1206, n.spec. = 3); 16-apr-2005, 2526); Paraćin: Sisevac (v.) (leg. G. Džukić, M. Kalezić s.d., 2638, n.spec. = 52); (leg. M. Kalezić 19-may-1978, 1009, n.spec. = 48); Peć: Gornje Barane (v.) (leg. V. Bušković 25-may-1984, 351, n.spec. = 1); Vitomirica (v.) (leg. H. Babić 10-jul1978, 351, n.spec. = 1); Pešter Plateau: Delimeđe (v.) (leg. anonim. 28-apr-2010, 2194, n.spec. $=$ 43); Petrovac Na Mlavi (leg. M. Radovanović s.d., 1469, n.spec. = 5); Pirot: Jerma river gorge, Vlasi (v.) (leg. J. Crnobrnja-Isailović, I. Aleksić 02-jun-1998, 1664, n.spec. =29); (2669); Kalna (v.), Vrtovac (v.) (leg. G. Džukić, M. Kalezić 18-jun-1996, 2551, n.spec. = 3); 15-may-2004, 1675); (leg. J. Crnobrnja-Isailović, I. Aleksić 01-jun-1998, 1648, n.spec. =1); Krupac (v.), Krupačko Blato lake (leg. D. Cvetković 28-apr-2000, 1231, n.spec. =3); Sukovo (v.) (leg. G. Džukić, M. Kalezić 17-jun-1980, 2590, n.spec. = 55); Požarevac: Mirijevo (v.), Tićevac (v.), Potez Vujkovac (leg. G. Džukić, M. Kalezić 17-apr-1994, 1647, n.spec. = 28); Tićevac (v.), Vujkovački Potok stream (leg. G. Džukić, M. Kalezić 17-apr-1994, 1518, n.spec. = 1); Preševo: Geren Dvor (leg. G. Džukić, M. Kalezić 11-may-1997, 1210, n.spec. = 2); Prijepolje: Jabuka (v.) (leg. G. Džukić 21-mar-1980, 1271, n.spec. = 1); Priština: Kosovo Polje (leg. Z. Martinović 1976, 351, n.spec. =1); Radevo (v.) (leg. M. Kalezić 01-may-1979, 1026, n.spec. = 23); Prizren: Gornje Selo (v.) (leg. R. Savić 11-jun-1978, 1608, n.spec. $=2$ ); Vrbnica (v.) (leg. R. Kaplan 03-apr-1976, 1608, n.spec. = 6); Raška: Aljinovići (v.) (leg. S. Marinković 07-aug-1991, 1307, n.spec. =15); Sombor: Svetozar Miletić (v.) (leg. G. Džukić, M. Kalezić 09-apr-1983, 1017, n.spec. $=40$ ); Sremska Mitrovica: Leget port (leg. anonim. 24-apr-1998, 210, n.spec. = 5); (leg. G. Džukić, M. Kalezić 24-apr-1998, 1670, n.spec. = 26); Šašinci (v.) (leg. G. Džukić 17-apr-1981, 2607, n.spec. =33); Zasavica (v.), Vrbovac and Pačija Bara (leg. G. Džukić, $M$. Kalezić 24-apr-1998, 1671, n.spec. =16); Srpska Crnja: Radojevo (v.) (leg. A. Rafeski 10-jul-1995, 2391, n.spec. =2); Stari Vlah: Sjenica (leg. G. Džukić, M. Kalezić 27-apr-2010, 2200, n.spec. =20); Uvac river, Sjenička cave (leg. anonim. 10-aug-1982, 1621, n.spec. $=7$ ); Subotica: Legrad (leg. anonim. 06-apr-1988, 2168, n.spec. = 10); Ludaško lake (leg. J. Mikuška 28-apr-1963, 1271, n.spec. = 6); Makova Sedmica (v.) (leg. G. Džukić, M. Kalezić 23-mar-1996, 1653, n.spec. = 5); Hrastovača (leg. G. Džukić 26-mar-1997, 1674, n.spec. = 1); (leg. G. Džukić, M. Kalezić 17-mar-1997, 1013, n.spec. = 31); (2622); 10-apr-1998, 1646); Hrastovača, Cirkuzantska Bara (leg. anonim. 02-oct-1998, 2420, n.spec. = 13); (leg. G. Džukić, M. Kalezić 23-mar-1996, 1653, n.spec. = 4); 08-mar-1998, 2612); Hrastovača, Crna Mlaka (leg. G. Džukić, M. Kalezić 07-mar-1998, 1692, n.spec. = 7); Hrastovača, Čavolj (leg. anonim. 08-mar-1998, 307, n.spec. = 19); (leg. G. Džukić, M. Kalezić 10-apr-1998, 1655, 
n.spec. = 22); 08-jul-1999, 2288); 15-oct-1999, 1233); (1669); Hrastovača, Pačarnik (leg. M. Kalezić, G. Džukić 24-mar-2000, 2112, n.spec. =7); Hrastovača, Pačarnik and Čavolj (leg. G. Džukić, M. Kalezić 23-mar-2000, 2299, n.spec. = 1); 23-may-2000, 1696); Subotica forests, Radanovačka forest (leg. G. Džukić, M. Kalezić 24-feb-1995, 1661, n.spec. = 5); Šabac: Orid (v.) (leg. G. Džukić, M. Kalezić 27apr-1998, 1652, n.spec. = 30); Trbušac (v.) (leg. G. Džukić, M. Kalezić 27-apr-1998, 1665, n.spec. = 10); (1806); Šid: Morović (v.) (leg. G. Džukić 16-apr-1981, 1023, n.spec. = 43); Trstenik: Velika Drenova (v.) (leg. G. Džukić, M. Kalezić 15-may-2000, 1213, n.spec. =9); Tutin: Mitrova (v.) (leg. G. Džukić, S. Cvetkov 20-may-1979, 1228, n.spec. = 48); Uroševac (leg. S. Novićević 15-oct-1976, 1608, n.spec. = 2); Užice: Mačkat (v.) (leg. G. Džukić 02-jun-1991, 2611, n.spec. = 29); Valjevo: Bujačić (v.) (leg. G. Džukić 21-may-1972, 1271, n.spec. =2); Bukovac (v.) (leg. G. Džukić 25-may-1980, 2579, n.spec. = 63); Debelo Brdo (leg. anonim. s.d., 1834, n.spec. =10); Đurđevac (v.) (leg. G. Džukić, M. Kalezić 16-apr-2005, 1221, n.spec. = 5); Gola Glava (v.) (leg. G. Džukić, M. Kalezić 24-apr-1998, 1657, n.spec. = 12); Gradac river gorge (leg. D. Ćirović 25-may-1993, 1618, n.spec. = 2); Joševa (v.) (leg. G. Džukić, M. Kalezić 26-apr-1998, 1803, n.spec. =3); 07-apr-2005, 1209); Mionica (leg. G. Džukić, M. Kalezić 16-apr-2005, 1224, n.spec. =4); Gornji Mušić (v.) (leg. G. Džukić 29-may-1980, 2577, n.spec. =3); Rakari (v.) (leg. G. Džukić 29-may-1980, 2578, n.spec. =3); Petnica (v.), Rogljević (v.) (leg. G. Džukić, M. Kalezić 31-may-1997, 1226, n.spec. = 1); Velika Plana: Žabari (v.) (leg. G. Džukić, M. Kalezić 24-may-1998, 1659, n.spec. = 29); Veliko Gradište: Kumane (v.) (leg. G. Džukić, M. Kalezić 14-apr-1994, 1643, n.spec. = 6); Vladimirci: Debrc (v.), Mićina, Nikolića Bara, Đurđina, Vejinovića, Đokina Bara (leg. G. Džukić 14-may-1993, 1667, n.spec. =87); Vlasina (leg. G. Džukić 23apr-2005, 2279, n.spec. = 1); Biljine Vode (leg. anonim. 23-jun-2007, 2229, n.spec. = 1); Dejanova river (leg. anonim. 24-jun-2010, 718, n.spec. =1); Đumurkana (leg. G. Džukić 20-may-1981, 2570, n.spec. = 39); Klisura (v.) (leg. G. Džukić, M. Kalezić 16-jun-2006, 2308, n.spec. =1); Vranje (leg. G. Džukić, M. Kalezić 14-may-1980, 2599, n.spec. = 70); Markovo Kale (leg. anonim. 14-may-1980, 2133 , n.spec. =4); Vršac: Vatin (v.) (leg. G. Džukić, M. Kalezić 07-may-2004, 1211, n.spec. = 5); Zaječar (leg. G. Džukić 18-jun-1980, 2716, n.spec. = 10); Grljan (v.), Prličko Blato (leg. G. Džukić, M. Kalezić 25-apr-2009, 1078, n.spec. = 1); Zlatibor (leg. Lj. Janković 1966, 2718, n.spec. = 1); Zlot (leg. J. Crnobrnja-Isailović, I. Aleksić 17-jun-1996, 1335, n.spec. = 11); Zrenjanin: Sečanj (v.), Neuzina (v.) (leg. anonim. 16-may-2003, 2472, n.spec. = 5); Slovenia (leg. anonim., s.d., 714, n.spec. = 1); Ajdovščina: Štanjel (v.), Kobjeglava (leg. G. Džukić, M. Kalezić 03-apr-1988, 2602, n.spec. = 39); (leg. J. Gregori, B. Kryštufek 07-may-1986, 726, n.spec. = 6); Ljubljana: Velike Lašče (v.), Podstrmec (v.) (leg. G. Džukić, M. Kalezić 04-jun-1981, 2373, n.spec. = 52); Murska Sobota: Radenci (v.) (leg. G. Džukić, M. Kalezić 01-apr-1988, 2596, n.spec. = 39); Piran: Sečovlje (v.) (leg. anonim. 1988, 1319, n.spec. =1); Rogaška Slatina: Gostišče Bellevue (leg. G. Džukić, M. Kalezić 26-may-1987, 2251, n.spec. $=8$ ).

\section{Lyciasalamandra fazilae}

Turkey Tersane Island: Gocek, Mugla (leg. N. Uzun, s.d., 2423, n.spec. =14).

\section{Neurergus strauchii}

Turkey Malatya: Tephan, Uzuntas (v.) (leg. N. Uzun, s.d., 2424, n.spec. = 10).

\section{Ommatotriton ophryticus}

Turkey Kostamonu: Tosya (leg. N. Uzun, s.d., 2786, n.spec. = 19).

\section{Salamandra atra}

Serbia Mts Prokletije: Mt. Bogićevica (leg. anonim., 12-sep-1996, 286, n.spec. =2); Maja Rops, Pasji Vrh (leg. anonim. s.d., 280, n.spec. $=2$ ); Raški Do (leg. anonim. s.d., 284, n.spec. $=1$ ); Slovenia Kranj: Mt. Snežnik Kranjski (leg. anonim., 20-jul-1982, 279, n.spec. =10); Ptuj: Dolič (v.) (leg. M. Radovanović 01-aug-1950, 1510, n.spec. =1). 


\section{Salamandra salamandra}

S.l. (leg. anonim., s.d., 271, n.spec. = 3); (272); 273); (274); 275); (270); 277); (278); (283); 287); (304); 322); (324); 326); (540); 546); (549); 695); (1275); 1278); (1308); 1309); (1447); 2215); (2689); (leg. G. Džukić s.d., 1304, n.spec. = 1); Bosnia and Herzegovina Foča: Brod (v.) (leg. S. Marinković, 23-mar-1989, 1474, n.spec. =1); Rijeka: Mamići (v.), Macanova Lokva puddle (leg. anonim. 08-apr-1989, 1428, n.spec. =2); Croatia Gospić: Smiljan (v.) (leg. G. Džukić, 03-jun-1985, 2267, n.spec. $=47$ ); Krbavsko Polje: Udbina (v.), Studenac (wellhead) (leg. anonim. 07-aug1968, 2682, n.spec. = 8); Makarska: Tučepi (v.) (leg. anonim. 11-aug-1968, 287, n.spec. =3); Mt. Dinara: Tijarica (v.), Vukići, Jelinac, Turkey puddle (leg. G. Džukić 04-jun-1985, 1628, n.spec. $=$ 4); Mt. Velebit: Oštarije (v.) - Brušane (v.) (leg. G. Džukić 12-apr-1989, 268, n.spec. = 2); Zagreb: Čučerje (v.), Bunar (leg. Petrić 20-apr-1975, 693, n.spec. = 1); Macedonia Mt. Jakupica (leg. G. Mesaroš, 07-jun-1988, 1446, n.spec. = 2); Montenegro Berane [“Ivangrad"]: Petnjica (v.) (leg. anonim., s.d., 1275, n.spec. =3); Polica (leg. R. Celić 20-may-1982, 282, n.spec. = 1); Gragani (leg. V. Ivanović 18-nov-2000, 1276, n.spec. =1); Kolašin: Lipovo (v.) (leg. anonim. s.d., 1275 , n.spec. = 3); Topli Potok (v.) (leg. anonim. 10-jun-1949, 2530, n.spec. =12); Mt. Bjelasica: Biogradsko lake (leg. G. Džukić 11-jul-1996, 1285, n.spec. = 4); Mt. Durmitor (leg. anonim. s.d., 1314, n.spec. =1); Mt. Rumija: Sutorman (v.), Lera (leg. G. Džukić, M. Kalezić 02-jun-1990, 1523, n.spec. = 5); Ublić (leg. S. Vuletić 01-may-1992, 652, n.spec. = 1); Mt. Žijovo: Bukumirsko lake, Donje lake (leg. M. Cirović 18-jun-1997, 1650, n.spec. =2); Rikavačko lake (leg. anonim. 10-aug2000, 1289, n.spec. =1); (leg. M. Perković, R. Ćirović 22-aug-2000, 1277, n.spec. =1); Mts Prokletije: Grbaja (leg. anonim. 01-jul-1984, 2726, n.spec. =1); Piperi: Piperske Stene (leg. G. Džukić 08may-1992, 2782, n.spec. = 1); Skadarsko lake: Krajina, Kostanica (v.) (leg. anonim. 21-apr-1991, 2770, n.spec. =1); Livari (v.) (leg. anonim. s.d., 1457, n.spec. = 3); Tara river canyon: Tepca (v.) (leg. G. Mesaroš 27-jun-1988, 1079, n.spec. = 1); Bajlovića Sige (leg. anonim. 22-jul-1988, 2774, n.spec. = 4); Serbia Bor: Krivelj (v.) (leg. anonim., 15-sep-1979, 1249, n.spec. = 3); Despotovac: Resava canyon (leg. B. Žiljak 20-apr-1996, 1258, n.spec. =19); Dimitrovgrad: Sv. Dimitar monastery (leg. S. Cvetkov 12-may-2005, 2758, n.spec. =15); Golubac: Ridanj (v.) (leg. Stošić, G. Džukić 20-oct-1982, 277, n.spec. =1); Grdelička gorge: Predejane (v.), Predejanska river (leg. G. Džukić, M. Kalezić 31-mar-2007, 1039, n.spec. =12); Vladičin Han, Džep (v.), Džepska river (leg. G. Džukić, M. Kalezić 19-apr-2007, 2145, n.spec. =1); Kosovska Mitrovica: Leposavić, Postenje (v.) (leg. J. Crnobrnja-Isailović, I. Aleksić s.d., 1252, n.spec. = 1); Kraljevo: Beranovac (v.) (leg. anonim. s.d., 1275, n.spec. =2); Loznica: Zajača (v.) - Paskovac (v.) (leg. G. Džukić 17-apr-1990, 2745, n.spec. = 5); Ljubovija: Gornja Trešnjica (v.), Popovići (leg. S. Marinković 14-apr-2000, 592, n.spec. = 3); Trešnjica canyon (leg. S. Marinković 21-apr-1991, 1443, n.spec. =1); Mt. Avala (leg. G. Džukić, M. Kalezić 01-may-2000, 1257, n.spec. = 3); Markovići (v.) (leg. T. Vukov, M. Slijepčević, A. Urošević, N. Tomašević-Kolarov 01-apr-2014, 2762, n.spec. =10); Mt. Beljanica: Lisine waterfall (leg. anonim. 06-oct-2006, 2234, n.spec. =1); Mt. Besna Kobila: Kriva Feja (v.), Nesvrta (v.) (leg. G. Džukić, M. Kalezić 25-sep-2005, 2154, n.spec. = 3); Mt. Boranja (leg. G. Mesaroš 10-jun-1987, 2769, n.spec. =1); Mt. Čemernik: Brankovci (v.) (leg. anonim. 26-sep-2005, 1825, n.spec. =1); Pečurka (leg. G. Džukić, M. Kalezić 26-sep-2008, 2180, n.spec. =2); Mt. Kopaonik: Samokovska river gorge (leg. J. Crnobrnja-Isailović 01-may-1986, 1626, n.spec. =2); Mt. Kukavica: Đokini Virovi, Gneždo and Jasiško Zavište (leg. G. Džukić, M. Kalezić 16-jun-2001, 1099, n.spec. = 5); Krpejce (v.) (leg. G. Džukić 18-sep-2003, 1837, n.spec. = 5); Mt. Maljen (leg. V. Kalafatić 19-mar-1991, 281, n.spec. $=1$ ); Mt. Medvednik (leg. anonim. s.d., 1275, n.spec. =1); Mt. Miroč: Cvetanovačka Bara (leg. G. Džukić, M. Kalezić 17-may-2000, 1202, n.spec. =2); Mt. Ostrozub: Ruplje (v.) (leg. G. Džukić, M. Kalezić 26-sep-2008, 2180, n.spec. =1); Mt. Radan: Đavolja Varoš, Zebice (v.) (leg. G. Džukić 21may-1977, 1080, n.spec. = 1); Mt. Stara Planina: Ilijina river (leg. P. Jakšić 11-jul-1991, 1222, n.spec. = 2); Mt. Tara: Rača (v.), Rača monastery (leg. G. Džukić 13-mar-1981, 277, n.spec. =1); Mt. Vidlič: Rsovci (v.), Sveti Ilija monastery (leg. G. Džukić, M. Kalezić 26-sep-2008, 2180, n.spec. = 2); Mt. Vršačke Planine (leg. G. Džukić, M. Kalezić 12-apr-2004, 2309, n.spec. = 3); Široko Bilo (leg. anonim. 25-oct-1994, 1254/1, n.spec. = 22); 1254/2); Mts Šar-Planina: Brezovica (v.), Sevce (v.) (leg. anonim. 1994, 1837, n.spec. = 3); (leg. M. Sarić 01-jun-1980, 282, n.spec. =1); (leg. S. Đorđević 17oct-1987, 282, n.spec. =1); Negotin: Tamnič (v.) (leg. Žikić 01-feb-1994, 1494, n.spec. =1); Novi 
Pazar (leg. R. Alić 10-jun-1987, 282, n.spec. = 1); Obrenovac: Umka (v.) - Barič (v.), Duboko (v.) (leg. M. Milenković 28-oct-2001, 1251, n.spec. =1); Paraćin: Grza river (leg. anonim. 07-may1976, 1460, n.spec. =9); (leg. M. Kalezić 07-aug-1976, 249, n.spec. = 9); Peć (leg. Dž. Memić 10-jul1984, 282, n.spec. = 1); Pirot: Kalna (v.), Inovo (v.) (leg. G. Džukić, M. Kalezić 11-apr-2008, 1253/1, n.spec. = 10); 1253/2); Mt. Vidlič, Rsovci (v.), Rsovci mountain cottage (leg. G. Džukić, M. Kalezić 25-sep-2008, 1053, n.spec. = 2); Temska (v.), Cerova (v.) (leg. G. Džukić, M. Kalezić 11-apr-2008, 1357, n.spec. = 1); Stanjinac - Cerova, Ripaljka (leg. G. Džukić, M. Kalezić 25-sep-2008, 1053, n.spec. = 1); Visočka Ržana (v.) (leg. G. Džukić, M. Kalezić 26-sep-2008, 2180, n.spec. = 1); Zvonačka Banja spa, Kusa Vrana (v.) (leg. anonim. 01-oct-2005, 1256, n.spec. =1); Požarevac: Tićevac (v.), Vujkovački Potok stream (leg. G. Džukić, M. Kalezić 17-apr-1994, 1518, n.spec. = 16); Preševo: Borovac (v.) (leg. G. Džukić, M. Kalezić 21-may-2008, 2538, n.spec. =1); Priština: Mt. Grmija (leg. B. Bajčetić s.d., 282, n.spec. = 1); (leg. O. Papović 12-jun-1982, 1033, n.spec. = 2); Prizren: Sredska (v.) (leg. G. Pasuljević 16-sep-1981, 282, n.spec. =1); Sićevačka gorge: Oblik - Ostrovica (v.) (leg. Lj. Tomović, D. Jović 05-jul-1997, 288, n.spec. =11); Svrljig: Periš (v.), Miranovačka Kula (leg. G. Džukić, M. Kalezić 11-apr-2008, 1250, n.spec. =1); Samar cave (leg. anonim. s.d., 323, n.spec. =10); Uroševac: Vitina, Žitinje (v.) (leg. M. Urošević 26-oct-1987, 282, n.spec. = 1); Valjevo (leg. A. Hegediš 05-jun-1996, 2682, n.spec. = 4); Bukovac (v.), Oreovac (leg. G. Džukić, M. Kalezić 29-aug2004, 2146, n.spec. = 6); Debelo Brdo (leg. anonim. s.d., 1275, n.spec. = 1); Degurić (v.) (leg. anonim. 07-jul-1949, 1467, n.spec. = 5); Đurđevac (v.) (leg. G. Džukić, M. Kalezić 16-apr-2005, 1221, n.spec. = 1); Gradac river gorge (leg. D. Ćirović 25-may-1993, 1618, n.spec. = 1); Valjevska Kamenica (v.) (leg. M. Kalezić 30-apr-1990, 1635, n.spec. = 3); Vlasina (leg. G. Džukić 23-apr-2005, 2279, n.spec. = 1); Biljine Vode (leg. G. Džukić, M. Kalezić 23-jun-2007, 2229, n.spec. = 5); Ćumruk (leg. G. Džukić, M. Kalezić 26-sep-2008, 2180, n.spec. =2); Kalna (v.) - Gradska (v.) (leg. G. Džukić, M. Kalezić 27-sep-2008, 1074, n.spec. = 1); Kalna (v.) - Preslap (v.) (leg. G. Džukić, M. Kalezić 21sep-2007, 1266, n.spec. = 4); Ljutež (v.) (leg. G. Džukić, M. Kalezić 19-apr-2007, 2149, n.spec. = 1); Vranje: Mt. Krstilovica, Devotin, lake (leg. M. Kalezić, G. Džukić, M. Marković 24-sep-2005, 2558, n.spec. = 1); Zaječar: Vlaški Do (v.) (leg. G. Džukić 19-jun-1980, 1051, n.spec. = 1); Slovenia Kamnik: Kamniška Bistrica (leg. G. Džukić, 08-nov-1969, 322, n.spec. = 1); Kranj: Sveti Jošt (v.) (leg. B. Kryštufek, G. Džukić 26-may-1972, 1616, n.spec. = 3); Novo Mesto: Mirna (v.), Zabrđe (leg. B. Kryštufek 23-apr-1986, 714, n.spec. =2); Radovljica: Stara Fužina (leg. anonim. 26-sep1989, 691, n.spec. = 2).

\section{Taricha granulosa}

USA Lily lake (leg. Marin co., s.d., 679, n.spec. = 40); Sonoma lake (leg. D. Hedgecock, M. Kalezić 08-aug-1976, 1340, n.spec. =6).

\section{Triturus carnifex}

Croatia Istra: Motovun, Istarske Toplice spa (leg. G. Džukić, A. Ivanović, D. Bejaković, 31-mar1991, 2411, n.spec. = 43); Salakovci (v.) (leg. anonim. s.d., 2387, n.spec. = 40); Svetvinčenat (v.), Velika Vala (leg. G. Džukić, A. Ivanović, D. Bejaković 01-apr-1991, 2410, n.spec. = 40); Koprivnica: Peteranec (v.) (leg. anonim. 05-apr-1988, 715, n.spec. =2); Krk (ins.): Diviska (v.) (leg. anonim. 1990, 2386, n.spec. = 1); Otočac: Švica (v.) (leg. G. Džukić, M. Kalezić 01-jun-1980, 2389, n.spec. = 19); 12-apr-1989, 1394); (leg. M. Kalezić 01-apr-1979, 2451, n.spec. = 9); Žumberak (leg. anonim. 01may-1987, 2409, n.spec. = 34); Slovenia Ajdovščina: Štanjel (v.), Kobjeglava (leg. G. Džukić, $M$. Kalezić, 03-apr-1988, 2408, n.spec. = 28); Vipava, Goče (leg. G. Džukić, M. Kalezić 03-apr-1988, 2407, n.spec. = 30); Ljutomer (leg. G. Džukić 31-mar-1988, 723, n.spec. = 3); Murska Sobota: Hrastje Mota (v.), Turjanci (v.) (leg. G. Džukić, M. Kalezić 26-may-1987, 2251, n.spec. =1); Radenci (v.) (leg. G. Džukić, M. Kalezić 01-apr-1988, 2416, n.spec. = 23); Pohorje: Lovrenška lakes (leg. anonim. 1986, 714, n.spec. = 2); Ribnica: Podstrmec (v.) (leg. anonim. s.d., 2453, n.spec. = 19); (leg. G. Džukić, M. Kalezić s.d., 83, n.spec. = 18); Rogaška Slatina: Gostišče Bellevue (leg. G. Džukić, M. Kalezić 26-may-1987, 2251, n.spec. =1). 


\section{Triturus cristatus}

Poland Mogielica (leg. J. Szymura, 01-may-1979, 105, n.spec. $=27$ ); $(2415)$; Serbia Bela Crkva: Kruščica (v.) (leg. G. Džukić, M. Kalezić, 18-mar-2001, 2554, n.spec. =26); (leg. M. Kalezić, G. Džukić, M. Marković 20-may-1998, 2548, n.spec. = 9); Kusić (v.) - Kaluđerovo (v.), Leskovačko lake (leg. G. Džukić 20-may-1998, 2399, n.spec. = 23); Lug stream (leg. G. Džukíc, M. Kalezić 14-mar1999, 2549, n.spec. = 23); Mt. Miroč (leg. G. Džukić, M. Kalezić 20-jun-2007, 2203, n.spec. =11); 17may-2000, 20); 17-may-2009, 2413); (leg. M. Kalezić, G. Džukić 20-apr-2006, 2113, n.spec. =12); Brza Palanka (v.), Kilome (v.) (leg. G. Džukić, M. Kalezić 01-apr-2009, 1066, n.spec. =5); Stara Brza (v.), Balta Prun (leg. anonim. 22-apr-2005, 2356, n.spec. =1); (leg. M. Kalezić, G. Džukić 15-jul-1998, 236, n.spec. = 8); Mt. Vršačke Planine (leg. G. Džukić, M. Kalezić 12-apr-2004, 2561, n.spec. $=24$ ); 01-apr2006, 1070); hunting lodge (leg. G. Džukić, M. Kalezić 12-apr-2004, 2400, n.spec. =2); Mesić (v.) (leg. anonim. s.d., 2667, n.spec. = 38); (leg. G. Džukić 27-dec-1991, 2679, n.spec. = 36); (leg. M. Kalezić, G. Džukić, M. Marković 01-oct-1996, 2548, n.spec. =1); Negotin (leg. anonim. 21-apr-1988, 2406, n.spec. = 56); (leg. G. Džukić, M. Kalezić 21-apr-1988, 21, n.spec. = 63); (leg. J. Crnobrnja-Isailović, I. Aleksić 30may-1998, 2669, n.spec. = 4); Sikole (v.) (leg. anonim. 21-jun-2003, 2471, n.spec. = 20); Štubik (v.) (leg. anonim. 01-jun-1986, 2468, n.spec. = 35); (leg. G. Džukić, M. Kalezić 21-apr-1988, 2557, n.spec. = 22); (leg. J. Crnobrnja-Isailović, I. Aleksić 31-may-1998, 2669, n.spec. =1); (leg. M. Kalezić 17-jun-1996, 1442, n.spec. = 5); Zaječar: Trnavac (v.), Majino Okno (leg. M. Slijepčević, A. Urošević, G. Džukić, M. Kalezić 23-may-2012, 1329, n.spec. = 11); Zlot: Manastirište (leg. anonim. 18-jun-1996, 2547, n.spec. =9); Ukraine Zakarpatskaya Province: Kamenskoe (v.) (leg. anonim., 2000, 573, n.spec. =30).

\section{Triturus cristatus complex}

S.I. (leg. anonim., 23-mar-1993, 238, n.spec. = 5); 19-apr-2008, 2350); 24-apr-2008, 2348); 25 apr-2008, 2351); s.d., 327); (357); 708); (709); 711); (717); 1005); (1264); 1267); (1348); 1367); (1368); 1606); (2212); 2347); (2639); 2737); (2749); 2752); (2753); 2754); (2755); 2763); (2773); 2776); (2780); (leg. G. Džukić s.d., 1476, n.spec. = 7); (2345); 2749); Rečica (leg. J. Crnobrnja-Isailović 15-aug-1996, 2318, n.spec. =1); Tabovci (leg. anonim. 17-may-1989, 1486, n.spec. =2); Croatia Kutina (leg. J. Hain, 24-apr-1990, 1344, n.spec. = 1); Zagreb: Sekirovo Selo (v.) (leg. anonim. 19may-1980, 2783, n.spec. =4); Serbia (leg. anonim., s.d., 1462, n.spec. =1).

\section{Triturus dobrogicus}

S.l. (leg. anonim., 20-apr-1996, 2757, n.spec. = 1); 06-may-2005, 2349); s.d., 683); (1366); 2187); (2240); Croatia Baranja: Bilje (v.) (leg. anonim., 14-apr-1972, 2755, n.spec. =1); Sisak: Mokro Polje (v.), Mlječko Polje (leg. I. Ham 27-apr-1990, 2388, n.spec. = 8); Slavonski Brod (leg. G. Džukić, M. Kalezić 08-apr-1988, 2417, n.spec. = 24); Velika Gorica: Turopolje, Orle (v.) (leg. G. Džukić 09-jun-1977, 2404, n.spec. = 47); Serbia Apatin: Forland (leg. anonim., 24-jul-1984, 1052, n.spec. = 1); Bela Crkva: Jasenovo (v.) (leg. G. Džukić, M. Kalezić 27-apr-2001, 2397, n.spec. = 33); Kaluđerovo (v.), Leskovački Potok stream (leg. G. Džukić, M. Kalezić 28-mar-2003, 1229, n.spec. = 6); Beograd: Dobanovci (v.) (leg. anonim. 29-mar-1995, 1859, n.spec. = 1); Makiš (leg. M. Radovanović 1949, 1148, n.spec. = 35); Ostružnica (v.) (leg. anonim. 14-jun-1986, 1316, n.spec. = 3); Topčider (leg. V. Tomić s.d., 1467, n.spec. = 3); Čoka (leg. anonim. 18-jun-1982, 1158, n.spec. =1); Deliblato sands: Đurica, Stevanove Ravnice (leg. anonim. 21-may-1998, 2555, n.spec. =21); (leg. G. Džukić, M. Kalezić 18-jul-2001, 2475, n.spec. =23); Majur Bara (leg. anonim. 09-apr-1981, 2755, n.spec. = 1); Utrine, Đabin Salaš (leg. G. Džukić, M. Kalezić 26-jun-1999, 1086, n.spec. =1); Zubanov Salaš (leg. G. Džukić 17-jun-1999, 2546, n.spec. = 3); 22-may-1999, 2542); (leg. M. Marković 30-apr2000, 1093, n.spec. =1); Kikinda (leg. G. Džukić, M. Kalezić 27-mar-2006, 1069, n.spec. = 3); Kovin (leg. anonim. 1970, 2755, n.spec. = 1); Novi Kneževac (leg. M. Kalezić, G. Džukić 21-may-1988, 235/2, n.spec. = 83); Novi Sad: Krčedin (v.), Krčedinska Ada (ins.) (leg. G. Džukić 30-apr-1980, 1089, n.spec. =1); Obedska Bara (leg. anonim. 10-may-1996, 1607, n.spec. =2); (leg. M. Kalezić, G. Džukić 01-may-1978, 2384, n.spec. = 31); Obrež (v.), Ravenica (leg. G. Džukić, M. Kalezić 30-apr-1992, 2353, n.spec. =1); 11-may-2004, 2396); Pančevo: Gradska Šuma forest (leg. M. Milenković 16-oct-1995, 1888, n.spec. =1); Ivanovo (v.) (leg. anonim. 29-mar-1980, 2499, n.spec. = 20); (leg. G. Džukić, M. 
Kalezić 02-apr-1980, 1, n.spec. = 16); (2553); Sombor: Svetozar Miletić (v.) (leg. anonim. s.d., 2390, n.spec. =62); (leg. G. Džukić, M. Kalezić 09-apr-1983, 4, n.spec. =62); Srem: Dobanovci (v.) (leg. G. Džukić, M. Kalezić 22-may-1993, 1402, n.spec. =3); Sremska Mitrovica: Kuzmin (v.) (leg. J. Simić 03-aug-1978, 1462, n.spec. =4); Leget port (leg. anonim. 24-apr-1998, 716, n.spec. =1); (leg. G. Džukić, M. Kalezić 24-apr-1998, 2392, n.spec. = 3); Zasavica (v.), Vrbovac and Pačija Bara (leg. G. Džukić, M. Kalezić 24-apr-1998, 1265, n.spec. = 1); Sremski Karlovci: Karlovački Vinogradi station (leg. A. Ivanović 07-jun-2015, 2806, n.spec. = 5); Srpska Crnja: Radojevo (v.) (leg. A. Rafeski 01-jul-1994, 1890, n.spec. = 1); 10-jul-1995, 2391); (leg. anonim. 06-jul-1995, 1511, n.spec. = 1); (2412); 14-jul-1995, 1511); 11-sep-1995, 2395); Subotica: Makova Sedmica (v.), Hrastovača, Cirkuzantska Bara (leg. anonim. 02-oct-1998, 2420, n.spec. =4); (leg. G. Džukić, M. Kalezić 17-mar1997, 2393, n.spec. = 8); 08-mar-1998, 2394); Šabac: Orid (v.) (leg. G. Džukić, M. Kalezić 27-apr-1998, 2403, n.spec. =4); Trbušac (v.) (leg. anonim. 27-apr-1998, 2447, n.spec. = 26); Šid: Jamena (v.) (leg. M. Marković 16-apr-1981, 2425, n.spec. =18); Vršac: Vatin (v.) (leg. G. Džukić, M. Kalezić 07-may2004, 2398, n.spec. =9); Ukraine Solotvino: Batevo (leg. L.J. Borkin, 18-jun-1905, 964, n.spec. =20).

\section{Triturus ivanbureschi}

Macedonia Dojran: Dojransko lake (leg. G. Džukić, 24-jun-1998, 1006, n.spec. =1); Suva Reka accumulation (leg. G. Džukić 08-may-1997, 2552, n.spec. =16); (leg. G. Džukić, M. Kalezić 08may-1997, 2369, n.spec. = 2); Kumanovo - Kriva Palanka: Stracin (v.) (leg. G. Džukić, M. Kalezić 10-may-1997, 36, n.spec. = 17); (leg. G. Džukić, S. Petkovski 24-jun-1995, 215, n.spec. =4); Mokro lake (leg. G. Džukić 25-may-2004, 1826, n.spec. =1); Suvo lake (leg. anonim. 10-may-1997, 2559, n.spec. = 17); Mt. Kožuf: Visoka Čuka (leg. anonim. s.d., 2462, n.spec. = 39); Mt. Osogovske Planine: Ponikva (leg. G. Džukić, S. Petkovski 23-jun-1995, 215, n.spec. = 1); Probištip: Lesnovo (v.) (leg. anonim. 1989, 2465, n.spec. =27); Serbia Bosilegrad: Brankovci (v.), Zli Dol (v.) (leg. G. Džukić, M. Kalezić, M. Denoel, 26-sep-2005, 2550, n.spec. = 8); Dimitrovgrad: Borovsko Polje, Velika Lokva puddle (leg. anonim. 10-apr-2008, 1821, n.spec. =1); Jagodina: Bunar (v.) (leg. B. Stanković 1990, 2429, n.spec. = 1); Mt. Avala: Trešnja (leg. anonim. 26-apr-1999, 1603, n.spec. = 1); (leg. G. Džukić 1981, 2469, n.spec. = 40); (leg. G. Džukić, M. Kalezić 1981, 17, n.spec. = 45); Mt. Čemernik: Bele Rovine (leg. G. Džukić, M. Kalezić 24-jun-2007, 2172, n.spec. =1); Mlačište (v.), Kesino lake (leg. G. Džukić, M. Kalezić 15-jun-2006, 2541, n.spec. =1); Pirot: Berovičko lake (leg. G. Džukić, M. Kalezić 14-sep-2006, 2545, n.spec. = 12); (2675); Jerma river gorge, Vlasi (v.) (leg. anonim. s.d., 2118 , n.spec. =2); (leg. M. Slijepčević, A. Ivanović 04-apr-2013, 1868, n.spec. =1); Kalna (v.), Vrtovac (v.) (leg. anonim. 18-jun-1996, 2401, n.spec. =21); (leg. G. Džukić, M. Kalezić 18-jun-1996, 2551, n.spec. = 3); (leg. J. Radojičić 18-jun-1996, 2544, n.spec. = 1); Vlasina: Stojkovića Mahala (v.) (leg. anonim. s.d., 32, n.spec. =36); (2562); Turkey Afion (leg. N. Uzun, s.d., 2337, n.spec. =3).

\section{Triturus karelinii}

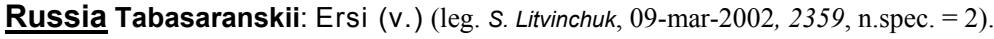

\section{Triturus macedonicus}

S.I. (leg. anonim., 19-apr-2008, 2355, n.spec. = 4); 21-apr-2008, 2354); 13-may-2010, 2338); s.d., 2346); (2496); Dobri Do (leg. R. Šupić 12-apr-1980, 1462, n.spec. =1); Bosnia and Herzegovina Tuzla: Dubrave (v.) (leg. anonim., s.d., 2405, n.spec. = 39); Macedonia Bitolj: Rotinske accumulation (leg. Bio Eko, 24-may-2002, 2436, n.spec. =6); Mavrovo (leg. Monveilleis 15-may1997, 2764, n.spec. = 1); Mt. Galičica (leg. anonim. s.d., 2446, n.spec. = 40); Kostobačilo (leg. anonim. 14-jun-1981, 2445, n.spec. = 35); (leg. G. Džukić, M. Kalezić 14-jun-1981, 54, n.spec. = 35); Mečkina Lokva puddle (leg. anonim. 14-jun-1981, 2454, n.spec. = 35); Mala Lokva (leg. anonim. 07may-1997, 2450, n.spec. = 42); Mt. Jakupica (leg. G. Mesaroš 07-jun-1988, 1446, n.spec. = 5); Prespa lake (leg. anonim. 18-may-1980, 2463, n.spec. = 34); Ezerani (v.) (leg. G. Džukić 07-may1977, 2441, n.spec. = 3); Prilep (leg. anonim. 15-may-1980, 2455, n.spec. =46); Skoplje: Petrovec (v.) (leg. anonim. 23-may-1981, 2464, n.spec. = 57); (leg. M. Kalezić 23-sep-1981, 202, n.spec. = 10); 
Struga: Struško Pole, Misleševo (v.) (leg. G. Džukić 07-may-1997, 2426, n.spec. = 3); Štip (leg. anonim. 1958, 1289, n.spec. =1); Montenegro Cetinje: Bjeloši (v.) (leg. anonim., 30-may-1990, 2452, n.spec. $=45$ ); Lašor puddle (leg. G. Džukić, M. Kalezić 30-may-1990, 1040, n.spec. = 1); Ćeklići, Kućišta (v.), Crno Korito (leg. M. Kalezić, G. Džukić, M. Marković 20-jun-2004, 2440, n.spec. =1); Kućišta (v.), Ublica (leg. M. Kalezić, G. Džukić, M. Marković 20-jun-2004, 2523, n.spec. =9); Dobrsko (v.) (leg. anonim. 04-apr-1989, 2460, n.spec. = 38); Rid (leg. G. Džukić, M. Kalezić 13-mar-2006, 1056, n.spec. = 1); Dobrsko (v.) (leg. G. Džukić, M. Kalezić 04-apr-1989, 2556, n.spec. =1); Danilovgrad: Lazine, Kosić (leg. G. Džukić, M. Kalezić 26-apr-2002, 2444, n.spec. =11); Lješanska Nahija (leg. anonim. s.d., 2501, n.spec. = 44); Mt. Bjelasica: Biogradsko lake, Jezerštica (leg. M. Kalezić, G. Džukić 25-aug-1994, 223, n.spec. =1); Mt. Lovćen (leg. anonim. 28-may-1990, 2461, n.spec. =27); (leg. G. Džukić 14-aug-1984, 2458, n.spec. = 7); Bijeloši (v.), Lašor (leg. G. Džukić, M. Kalezić 30-may1990, 1827, n.spec. =1); Plav: Brezojevica (v.) (leg. G. Pasuljević 01-aug-1974, 1462, n.spec. =1); Pula (leg. G. Pasuljević 01-aug-1974, 1462, n.spec. =1); Podgorica: Draževina (v.), Kamenica (leg. G. Džukić, M. Kalezić 05-jun-2005, 2434, n.spec. =2); Lipa puddle (leg. M. Kalezić, G. Džukić 22-jul1997, 250, n.spec. =1); Seoski Ubao (leg. G. Džukić, M. Kalezić 22-aug-2001, 2430, n.spec. = 2); Progonovići (v.) (leg. M. Kalezić, G. Džukić 23-mar-2007, 2115, n.spec. =2); Stabanovi Gornji (leg. G. Džukić, M. Kalezić 22-aug-2001, 2427, n.spec. =2); Tuzi (v.), Mt. Dečić (leg. anonim. 29-may-1996, 1452, n.spec. =1); (1506); Rijeka Crnojevića: Ceklin (v.) (leg. anonim. 26-may-1996, 208, n.spec. = 2); (leg. G. Džukić, M. Kalezić 29-apr-1992, 2256, n.spec. = 20); (leg. G. Džukić, M. Kalezić, A. Ivanović s.d., 2108, n.spec. =12); Brežina (v.), Donji Lokanj (leg. anonim. 17-sep-1990, 2467, n.spec. = 56); (2759); (leg. G. Džukić, M. Kalezić 08-jun-1990, 2253, n.spec. = 21); 17-sep-1990, 2672); 16-sep-1991, 2262); Brežina (v.), Lokanj (leg. anonim. 21-feb-1993, 2223, n.spec. = 3); 02-apr-1995, 205); 06-apr-1995, 203); 07-apr-1995, 1865); (leg. G. Džukić, M. Kalezić, A. Ivanović 01-sep-1991, 1840, n.spec. = 1); Pijavica spring (leg. G. Džukić, A. Ivanović, M. Kalezić 17-mar-2008, 2352, n.spec. =1); (leg. G. Džukić, M. Kalezić 1992, 2341, n.spec. = 6); Rid (leg. G. Džukić, M. Kalezić 13-mar-2007, 2182, n.spec. = 9); Strugari (leg. anonim. 19-sep-1991, 2189, n.spec. =1); Ceklin and Ljubotin (leg. anonim. 18-mar-2008, 1821, n.spec. =9); Ceklin and Progonovići (v.) (leg. G. Džukić, M. Kalezić 13-mar-2007, 2230, n.spec. = 4); Gornji Ceklin (v.) (leg. anonim. 19-sep-1981, 2456, n.spec. = 69); 23-dec-1991, 2457); (leg. G. Džukić, M. Kalezić 11-feb-1992, 2259, n.spec. =13); Pijavica spring (leg. G. Džukić, M. Kalezić 26-aug2002, 2302, n.spec. =1); (leg. G. Džukić, M. Kalezić, D. Bejaković 14-jul-1992, 1529, n.spec. = 3); Jankovića Krš (v.), Pištet (leg. anonim. 14-jul-1992, 1491, n.spec. =3); Jankovića Krš (v.) and Rid (leg. G. Džukić, M. Kalezić 19-jul-1992, 2250, n.spec. = 20); Skadarsko lake: Krajina, Livari (v.) (leg. J. Crnobrnja-Isailović, I. Aleksić 29-may-1996, 200, n.spec. = 1); Ulcinj: Donji Štoj (v.) (leg. anonim. 02-apr-1989, 2466, n.spec. = 35); (leg. G. Džukić, M. Kalezić 01-apr-1981, 1842, n.spec. =1); (leg. M. Marković, G. Džukić 23-jun-2004, 2331, n.spec. = 3); Serbia Aleksandrovac: Župa (leg. D. Rajković, 12-aug-1976, 1462, n.spec. =1); Grčak (v.) (leg. anonim. s.d., 2418, n.spec. =24); Avaje (Kosovo) (leg. V. Talović s.d., 1462, n.spec. =1); Gnjilane: Kosovska Kamenica, Ograđe (leg. B. Milošević 28-apr-1980, 1462, n.spec. =2); Grdelička gorge: Migulovac (v.) (leg. G. Džukić, M. Kalezić 18-apr-2007, 2435, n.spec. = 11); lake (leg. G. Džukić, M. Kalezić 18-apr-2007, 2431, n.spec. $=$ 11); Kosovo (leg. G. Pasuljević s.d., 1462, n.spec. $=4$ ); Kosovska Mitrovica: Leposavić, Dren stream (leg. R. Kostović, G. Pasuljević 06-may-1973, 1462, n.spec. =2); Kruševac (leg. ž. Cvetković 01may-1959, 1467, n.spec. = 10); Leskovac: Vučje, Manetove Livade (leg. G. Džukić, M. Kalezić 16jun-2001, 1105, n.spec. = 5); Todorovce (v.) (leg. G. Džukić, M. Kalezić 15-jun-2001, 2329, n.spec. =2); Todorovce (v.), Crepanski Virovi (leg. G. Džukić, M. Kalezić 04-may-2001, 46, n.spec. = 9); (2560); Ljubovija: Gledeće (v.) (leg. anonim. 05-apr-1998, 237, n.spec. =1); Mt. Kopaonik (leg. anonim. 28-may-1986, 2749, n.spec. = 2); Jošanička Banja spa (leg. anonim. 29-apr-1986, 2749, n.spec. $=$ 4); Radošiće (v.) (leg. anonim. 29-apr-1984, 2449, n.spec. = 38); 29-apr-1986, 2749); Mt. Maljen: Divčibare, Pecina Bara (leg. J. Crnobrnja-Isailović 24-jul-1999, 1103, n.spec. =2); Mt. Rtanj (leg. G. Džukić, M. Kalezić 24-jun-1980, 2676, n.spec. = 16); Mt. Tara: Beli Rzav lake (leg. G. Džukić, M. Kalezić 12-jun-2001, 1133, n.spec. =2); Niš: Mezgraja (v.) (leg. Z. Nikolić 14-jun-1977, 1462, n.spec. = 1); 27-sep-1977, 1462); Novi Pazar (leg. R. Alić 20-aug-1982, 1462, n.spec. =1); Novo Brdo: Bostane (v.) (leg. P. Jakšić 25-apr-1987, 999, n.spec. = 9); Peć (leg. anonim. 01-aug-1995, 1407, n.spec. = 1); (2438); (leg. Lj. Markovíc 20-sep-1976, 1462, n.spec. = 2); (leg. S. Bašanović 29-sep-1976, 1462, n.spec. =1); Banja Ilidža spa (leg. S. Bašanović 19-sep-1976, 1462, n.spec. =1); Đurakovac 
(v.), Osojane (v.) (leg. D. Lakušić 10-oct-1978, 1462, n.spec. = 1); Žač (v.) (leg. D. Lakušić 26-may1978, 1466, n.spec. = 2); Gornje Barane (v.) (leg. V. Bušković 25-may-1984, 1462, n.spec. = 2); Istok (v.) (leg. V. Šabović 15-may-1979, 1462, n.spec. = 2); Karagač (leg. anonim. 01-aug-1995, 1886, n.spec. = 3); 17-may-1996, 2437); 23-may-1996, 2432); Zatra (v.) (leg. H. Babačić 27-aug-1978, 1462, n.spec. =4); Pešter Plateau: Delimeđe (v.) (leg. G. Džukić, S. Cvetkov 1-may-1980, 1228, n.spec. =1); Stanovi, Karaiska Bara (leg. G. Džukić, M. Kalezić 28-apr-2010, 1021, n.spec. =7); Petrovac Na Mlavi (leg. M. Radovanović 25-apr-1959, 1468, n.spec. =2); Podujevo (leg. V. Dumanović 25-aug-1978, 1462, n.spec. = 8); Preševo (leg. anonim. 1989, 2459, n.spec. = 2); (leg. J. Crnobrnja-Isailović, I. Aleksić 05-jun1998, 2669, n.spec. = 3); Borovac (v.) (leg. J. Crnobrnja-Isailović, I. Aleksić 05-jun-1998, 2669, n.spec. =

5); Priština: Ajvalijski Potok stream (leg. G. Pasuljević and students 13-may-1983, 1895, n.spec. = 1); Čaglavica (v.) (leg. S. Ristić 20-may-1983, 1462, n.spec. =1); Gračanica (leg. Z. Jovanović 24oct-1980, 1462, n.spec. = 21); Janjevo (v.) (leg. M. Tolić 15-nov-1977, 1462, n.spec. = 1); Kosovo Polje (leg. D. Čukić 08-may-1983, 1895, n.spec. =3); (leg. M. Dimić 15-may-1981, 1895, n.spec. =1); (leg. M. Todorović s.d., 1462, n.spec. =1); Bresje (v.) (leg. Mladen D 15-apr-1983, 1408, n.spec. =1); Ugljare (v.) (leg. J. Martinović 03-oct-1976, 1462, n.spec. = 1); Mt. Grmija (leg. anonim. 13-jun-1995, 1008, n.spec. = 1); 29-may-1995, 206); (leg. G. Džukić 07-jun-1995, 2439, n.spec. = 2); 20-aug-1994, 2428); (leg. M. Kalezić 16-jun-1995, 1496, n.spec. =1); Obilić (leg. G. Pasuljević 01-apr-1967, 1004, n.spec. = 37); (01-apr-1967, 1141, n.spec. = 14); (1262, n.spec. = 37); Radevo (v.) (leg. M. Kalezić 01-may1979, 2442, n.spec. = 5); Priština - Uroševac: Lipljan (leg. V. Bijelić, G. Pasuljević 01-apr-1972, 1462, n.spec. = 11); Lipovica (v.), Slovinje (v.) (leg. anonim. s.d., 1462, n.spec. =7); Prizren: Gornje Selo (v.) (leg. R. Savić 10-may-1979, 1462, n.spec. =7); Lokvica (v.) (leg. M. Birdaini 07-sep-1978, 1462, n.spec. = 2); Soko Banja (leg. B. Jeftić 10-apr-1977, 1462, n.spec. =1); Svrljig: Samar cave (leg. anonim. s.d., 323, n.spec. = 1); Uroševac (leg. anonim. 10-apr-1976, 1462, n.spec. =17); 06-sep-1976, 1462); 15-nov-1976, 1462); 10-apr-1977, 1462); 09-oct-1976, 1462); (leg. B. Babić, B. Biserka 24-apr1977, 1462, n.spec. = 6); (leg. I. Hasković 20-jun-1977, 1462, n.spec. = 5); (leg. S. Novićević 15-oct-1976, 1462, n.spec. $=10)$; Nerodimlje (v.) (leg. anonim. 08-jul-1976, 1462, n.spec. =1); (leg. D. Marković 15jun-1980, 1462, n.spec. = 3); Valjevo: Bukovac (v.) (leg. anonim. 01-may-1980, 2414, n.spec. = 30); (leg. G. Džukić, M. Kalezić 01-may-1980, 55, n.spec. =9); Đurđevac (v.) (leg. G. Džukić, M. Kalezić 16apr-2005, 2117, n.spec. =4); Strinina Bara (leg. J. Crnobrnja-Isailović 28-jul-1999, 1204, n.spec. = 2); Gola Glava (v.) (leg. G. Džukić 26-apr-1998, 2433, n.spec. =2); Mionica (leg. J. Crnobrnja-Isailović 27-jul-1999, 1094, n.spec. = 3); Petnica (v.), Rogljević (v.) (leg. G. Džukić, M. Kalezić 31-may-1997, 2402, n.spec. =2); Rakari (v.) (leg. G. Džukić, M. Kalezić 29-may-1980, 2443, n.spec. =1); Vranje: Markovo Kale (leg. G. Džukić, M. Kalezić 14-may-1980, 61, n.spec. =12); Mt. Krstilovica, Devotin, lake (leg. M. Denoel, G. Džukić, M. Kalezić 24-sep-2005, 2543, n.spec. =19); (leg. M. Kalezić, G. Džukić, M. Marković 24-sep-2005, 2558, n.spec. =2); Mt. Sveti Ilija (leg. anonim. 14-may-1980, 2448, n.spec. = 12).

\section{Analysis of taxa and specimens}

This collection contains samples of 33 species from 18 countries. These species belong to 8 families ( 5 of Anura and 3 of Caudata).

\section{ANURA}

\section{Familia Bombinatoridae}

Specimens from this family belong to species Bombina bombina, $B$. variegata and hybrid of these two species B. bombina / B. variegata.

Bombina bombina - 1294 specimens from 3 countries (Serbia, Croatia and Bosnia and Herzegovina) in 97 entries with known localities, and 11 entries with no data. 
Bombina variegata - 2816 specimens from 7 countries (Serbia, Montenegro, Croatia, Macedonia, Bosnia and Herzegovina, Slovenia and Bulgaria) in 343 entries with known localities and 30 entries with no data.

B. bombina / B. variegata - 93 specimens from 1 country (Serbia) in 4 entries with known localities.

\section{Familia Pelobatidae}

Specimens of this family belong to species Pelobates fuscus and $P$. syriacus.

Pelobates fuscus - 330 specimens from three countries (Serbia, Croatia and Slovenia) in 67 entries with known localities, and 11 entries with no data.

Pelobates syriacus - 148 specimens from two countries (Macedonia and Serbia) in 29 entries with known localities.

\section{Familia Bufonidae}

Specimens of this family belong to species Bufo bufo and Bufo viridis.

Bufo bufo - 309 specimens from seven countries (Albania, Bosnia and Herzegovina, Montenegro, Serbia, Croatia, Slovenia and Macedonia) in 107 entries with known localities and 26 entries with no data.

Bufo viridis - 583 specimens from six countries (Bosnia and Herzegovina, Montenegro, Croatia, Macedonia, Serbia and Greece) in 149 entries with known localities and 23 entries with no data.

\section{Familia Hylidae}

Specimens of this family belong to species Hyla arborea and $H$. savignyi.

Hyla arborea - 457 specimens from six countries (Bosnia and Herzegovina, Montenegro, Croatia, Macedonia, Serbia and Greece) in 107 entries with known localities and 27 entries with no data.

Hyla savignyi - single specimen from one country (Iraq), in one entry with known locality.

\section{Familia Ranidae}

Specimens from this family belong to two genera: genus Pelophylax (water or green frogs) with species Pelophylax lessonae, P. kl. esculentus, $P$. ridibundus, $P$. shquipericus and $P$. kurtmuelleri and genus Rana (brown frogs) with species Rana arvalis, $R$. dalmatina, $R$. graeca and $R$. temporaria. Due to the difficulties in determination, hybridogenetic species of water frogs were often classified as $P$. esculentus complex. For instance, $P$. lessonae and $P$. kl. esculentus were, due to the extremely difficult morphological separation, classified as $P$. esculentus complex in this collection. Also, brown frogs which were problematic or difficult for 
determination (tadpoles, juveniles or individuals with unclear diagnostic traits) were classified as Ranae fuscae.

Pelophylax esculentus complex - 2209 specimens from seven countries (Bosnia and Herzegovina, Montenegro, Croatia, Macedonia, Slovenia, Serbia and Greece) in 228 entries with known localities and 71 entries with no data (these entries are in need of thorough revision, including genetic analyses, in order to correctly classify species).

Pelophylax kurtmuelleri - four specimens from one country (Macedonia) in three entries with known localities.

Pelophylax ridibundus - 126 specimens from six countries (Bosnia and Herzegovina, Montenegro, Croatia, Macedonia, Slovenia and Serbia) in 53 entries with known localities and six entries with no data.

Pelophylax shquipericus - 28 specimens from one country (Montenegro) in four entries with known localities.

Rana arvalis - 16 specimens from one country (Croatia) in four entries with known locality, and two entries with no data.

Rana dalmatina - 742 specimens from six countries (Bosnia and Herzegovina, Montenegro, Croatia, Macedonia, Slovenia and Serbia) in 234 entries with known localities and 36 entries with no data.

Rana graeca - 349 specimens from four countries (Bosnia and Herzegovina, Montenegro, Macedonia and Serbia) in 126 entries with known locality and 15 entries with no data.

Rana temporaria - 280 specimens from 7 countries (Bosnia and Herzegovina, Montenegro, Norway, Croatia, Macedonia, Slovenia and Serbia) in 97 entries with known locality and 21 entries with no data.

Ranae fuscae - 67 specimens from two countries (Montenegro and Serbia) in 17 entries with known locality and three entries with no data.

Girini - tadpoles which were problematic to identify -88 entries with known localities and 14 entries with no data.

\section{CAUDATA}

\section{Familia Proteidae}

Specimens of this family belong to species Proteus anguinus. - 4 specimens from 3 entries with no data.

\section{Familia Plethodontidae}

Specimens of this family belong to species Batrachoseps attennatus.

Batrachoseps attennatus - 5 specimens from one country (United States of America). 


\section{Familia Salamandridae}

Specimens of this family belong to 8 genera: Icthyosaura with species I. alpestris, Lissotriton with species $L$. helveticus, L. montadoni and $L$. vulgaris; Lyciasalamandra with one species L. fazilae; Neurergus with one species N. strauchii; Ommatotriton with one species O. ophryticus; Salamandra with two species $S$. atra and S. salamandra; Taricha with one species T. granulosa and Triturus with 5 species T. dobrogicus, T. carnifex, T. cristatus, T. ivanburechi, T. macedonicus. Due to the difficulties in determination, hybridogenetic species of crested newts were often classified as $T$. cristatus complex.

Icthyosaura alpestris - 6861 specimens from 6 countries (Bosnia and Herzegovina, Montenegro, Croatia, Macedonia, Serbia and Slovenia) in 308 entries with known locality and 38 entries with no data.

Lissotriton helveticus - 13 specimens from one country (France) in 5 entries.

Lissotriton montadoni - 19 specimens from two countries (Poland and Romania) in 2 entries with known data.

Lissotriton vulgaris - 9021 specimens from 7 countries (Bosnia and Herzegovina, Montenegro, Croatia, Macedonia, Serbia, Slovenia and Poland) in 462 entries with known locality and 35 entries with no data.

Lyciasalamandra fazilae - 14 specimens from one country (Turkey) in one entry with known data.

Neurergus strauchii - 10 specimens from one country (Turkey) in one entry with known data.

Ommatotriton ophryticus - 19 specimens from one country (Turkey) in one entry with known data.

Salamandra atra - 18 specimens from two countries (Serbia and Slovenia) in 6 entries with known data.

Salamandra salamandra - 566 specimens from 6 countries (Bosnia and Herzegovina, Montenegro, Croatia, Macedonia, Serbia and Slovenia) in 104 entries with known locality and 30 entries with no data.

Taricha granulosa - 46 specimens from one country (United States of America) in 2 entries with known data.

Triturus cristatus complex - 342 specimens from two countries (Serbia and Croatia) in 4 entries with known locality and 34 entries with no data.

Triturus dobrogicus - 656 specimens from three countries (Serbia, Croatia and Russia) in 53 entries with known locality and 6 entries with no data.

Triturus carnifex - 314 specimens from two countries (Croatia and Slovenia) in 18 entries with known locality. 
Triturus cristatus - 544 specimens from three countries (Serbia, Poland and Ukraine) in 30 entries with known locality.

Triturus ivanbureschi - 350 specimens from three countries (Serbia, Macedonia and Turkey) in 29 entries with known locality.

Triturus macedonicus - 1632 specimens from four countries (Serbia, Macedonia, Montenegro and Bosnia and Herzegovina) in 160 entries with known locality and 5 entries with no data.

Triturus karelinii - 2 specimens from one country (Russia) from one entry with known locality.

Girini - larvae which were problematic to identify - 3 entries with known localities and 2 entries with no data.

\section{DISCUSSION}

The Collection of Amphibians stored at the Institute for Biological research "Siniša Stanković", University of Belgrade, includes 30288 specimens from 33 species and 8 families. Best represented families are Salamandridae (20427 specimens), Bombinatoridae (4203) and Ranidae (3821) while families Proteidae and Plethodontidae are represented with few specimens. All confirmed species of Serbian Batrachofauna (Vukov et al. 2013) and most amphibian species characteristic for Balkan Peninsula are present in the collection.

This collection is distinctive because most of these species are covered with large population samples including those from the contact zones of closely related species: Bombina bombina and B. variegata (Arntzen 1978, Vukov et al. 2006); Triturus cristatus complex (Arntzen 2003, Arntzen et al. 2014) and Pelobates fuscus and P. syriacus (syntopy with no hybridization; Džukić et al. 2005). Also, we have a large population sample of green frogs (Pelophylax esculentus complex) from the contact zones in Pannonian plain and river valleys of the Danube, Sava and Velika Morava as well as Lake Skadar basin.

Paedomorphosis is a phenomenon characteristic for the tailed amphibians of Balkan karst (Denoël et al. 2001). Our collection is especially rich in samples of paedomophic newts, with large population samples.

The most valuable aspect of this batrachological collection is its taxonomical and geographical richness as well as the presence of large population series. The information stored in the Batrachological Collection at the Institute for Biological research "Siniša Stanković" besides its historical value, it's exceptionally valuable in the research opportunities it 
provides in systematics, biogeography, ecology, morphology, and many other aspects of the biology of amphibians. Also, with the presence of large population samples and different ontogenetic stages including hybrid zones, our collection has a potential contributing to the knowledge and better understanding of evolutionary phenomena.

Beside wet, alcohol stored Batrachological collection there is a collection of skeletal preparations which contain several hundred specimens that have been cleared and stained. The list of special, osteological collection will be published separately.

\section{Acknowledgements}

This research was partly financed by the Ministry of Education, Science and Technological Development of Republic of Serbia (Grant No. 173043). We thank J.W. Arntzen and Naturalis Biodiversity Center for logistic support. Also we would like to thank to all our colleagues and volunteers who helped us to make an inventory of batrachological collection.

\section{REFERENCES}

Arnold, E., Ovenden, D. (2002): A Filed Guide to the Reptiles and Amphibians of Britain and Europe. - Harper Collins Publishers, London.

Arntzen, J.W. (1978): Some hypotheses on postglacial migrations of the firebellied toad, Bombina bombina (Linnaeus) and the yellowbellied toad, Bombina variegata (Linnaeus). - Journal of Biogeography 5: 339-245.

Arntzen, J.W. (2003): Triturus cristatus Superspezies - Kammolch-Artenkreis. (Triturus cristatus (Laurenti, 1768) - Nördlicher Kammolch, Triturus carnifex (Laurenti, 1768) - Italienischer Kammolch. Triturus dobrogicus (Kiritzescu, 1903) - Donau-Kammolch, Triturus karelinii (Strauch, 1870) - Südlicher Kammolch). In: Grossenbacher K., Thiesmeier B. (ed.): Handbuch der Reptilien und Amphibien Europas 4/IIA: 421-514. - Wiebelsheim, AulaVerlag.

Arntzen, J.W., Wielstra, B., Wallis, G.P. (2014): The modality of nine Triturus newt hybrid zones assessed with nuclear, mitochondrial and morphological data. - Biological Journal of the Linnean Society 113: 604-622.

Denoël, M., Duguet, R., Džukić, G., Kalezić, M., Mazzoti, S. (2001): Biogeography and ecology of paedomorphosis in Triturus alpestris (Amphibia, Caudata). - Journal of Biogeography 28: 1271-1280.

Džukić, G. (1972): Herpetological collection of the Belgrade Museum of Natural History. - Zbornik Prirodnjačkog Muzeja, Ser. B 27: 165-180. In Serbian with English summary

Džukić, G. (1995): Diversity of amphibians and reptiles of Yugoslavia with the survey of species with international importance. In: Stevanovic, V., Vasic, V. 
(ed.): Biodiversity of Yugoslavia with the survey of species with International Importance. - Faculty of Biology and Ecolibri, Belgrade. In Serbian

Džukić, G., Kalezić, M.L. (2004): The Biodiversity of Amphibians and Reptiles in the Balkan Peninsula. In: Griffiths, H.I, Kryštufek, B., Reed, J.M. (ed.): Balkan Biodiversity: Pattern and Process in the European Hotspot: 167-192. Kluwer Academic Publishers, Dordrecht, Netherlands.

Džukić, G., Beškov, V., Sidorovska, V., Cogalniceanu, D., Kalezić, M.L. (2005): Historical and contemporary ranges of the spadefoot toads (Pelobates spp., Amphibia: Anura) in the Balkan Peninsula. - Acta zoologica cracoviensia 48A(1-2): 1-9.

Kalezić, M.L, Džukić, G., Mesaroš, G., Crnobrnja-Isailović, J. (1997): The crested newt (Triturus cristatus superspecies) in ex-Yugoslavia: morphological structuring and distribution patterns. - The university Thought 4: 39-46.

Krizmanić, I. (2008): Water frogs (Rana esculenta complex) in Serbia morphological data. - Archives of Biological Sciences, Belgrade 60: 449-457.

Krizmanić, I., Ivanović, A. (2010): Population systems of the Pelophylax esculentus complex in the southern part of its range. - Folia Zoologica 59: 214-221.

Vukov, T., Džukić, G., Lelo, S., Borkin, L.J., Litvinchuk, S.N., Kalezić, M.L. (2006): Multivariate morphometrics of the yellow-bellied toad (Bombina variegata) on the Central Balkans: taxonomical and biogeographical implications. - Zoological Studies 45: 213-222.

Vukov, T., Kalezić, M. L., Tomović, Lj., Krizmanić, I., Jović, D., Labus, N., Džukić, G. (2013): Amphibians in Serbia - Distribution and diversity patterns.

- Bulletin of the Natural History Museum 6: 90-112.

Wielstra, B., Crnobrnja-Isailović, J., Litvinchuk, S. N., Reijnen, B. T., Skidmore, A. K., Sotiropoulos, K., Toxopeus, A. G., Tzankov, N., Vukov, T., Arntzen, J.W. (2013): Tracing glacial refugia of Triturus newts based on mitochondrial DNA phylogeography and species distribution modeling. - Frontiers in Zoology 10: 13.

\section{SUPPORTING INFORMATION}

Online Appendix - List of amphibian specimens deposed in the batrachological collection with collection numbers, names of species, original locations, reconstructed and classified exact locations, date of collection, legators and number of specimens.

\section{ABBREVIATIONS IN THE CATALOGUE AND APPENDIX}

s.1. sine loco (without a place)

s.d. sine die (without a date) n.spec. number of specimens

v. village 


\section{БАТРАХОЛОШКА ЗБИРКА ИНСТИТУТА ЗА БИОЛОШКА ИСТРАЖИВАњА „СИНИША СТАНКОВИЋ“", УНИВЕРЗИТЕТА У БЕОГРАДУ}

ГЕОРГ ЏУКИЋ, МИЛЕНА ЦВИЈАНОВИЋ, АЛЕКСАНДАР УРОШЕВИЋ, ТАЮА Д. ВУКОВ, НАТАША ТОМАШЕВИЋ КОЛАРОВ, МАЈА СЛИЈЕПЧЕВИЋ, АНА ИВАНОВИЋ, МИЛОШ Л. КАЛЕЗИЋ

\section{Р Е 3 И М Е}

Овај рад представља попис врста водоземаца депонованих у батрахолошкој збирци Института за Биолошка Истраживања „Синиша Станковић“, Универзитета у Београду. Збирка се састоји од 33 врсте водоземаца сакупљених углавном на територији бивше Југославије, посебно из Србије. 